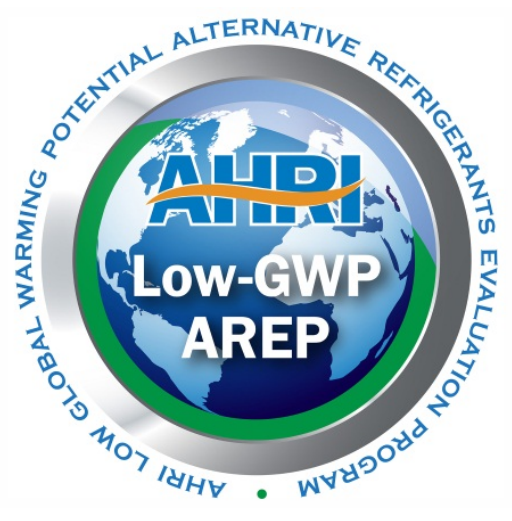

Air-Conditioning, Heating, and Refrigeration Institute (AHRI) Low-GWP Alternative Refrigerants Evaluation Program (Low-GWP AREP)

\title{
TEST REPORT \#21
}

\section{Compressor Calorimeter Test of R-404A Alternatives ARM-31a, D2Y-65, L-40, and R-32/R-134a (50/50)}

Som Shrestha,

Vishaldeep Sharma, And

Omar Abdelaziz

Oak Ridge National Laboratory

Oak Ridge, TN 3783

August 5, 2013

This report has been made available to the public as part of the author company's participation in the AHRI's LoW-GWP AREP.

\author{
1 10 \\ Air-Conditioning, Heating, and Refrigeration Institute \\ 2111 Wilson Boulevard, Suite 500 \\ Arlington VA 22201 \\ (703) 524-8800 \\ www.ahrinet.org
}


List of Tested Refrigerants' Compositions (Mass\%)

\begin{tabular}{|l|l|}
\hline ARM-31a & R-32/R-134a/R-1234yf (28/21/51) \\
\hline D2Y-65 & R-32/R-1234yf (35/65) \\
\hline L-40 & R-32/R-152a/R-1234yf/R-1234ze(E) (40/10/20/30) \\
\hline R-32/R-134a & R-32/R-134a (50/50) \\
\hline
\end{tabular}




\section{DISCLAIMER}

This report was prepared as an account of work sponsored by an agency of the United States Government. Neither the United States Government nor any agency thereof, nor any of their employees, makes any warranty, express or implied, or assumes any legal liability or responsibility for the accuracy, completeness, or usefulness of any information, apparatus, product, or process disclosed, or represents that its use would not infringe privately owned rights. Reference herein to any specific commercial product, process, or service by trade name, trademark, manufacturer, or otherwise, does not necessarily constitute or imply its endorsement, recommendation, or favoring by the United States Government or any agency thereof. The views and opinions of authors expressed herein do not necessarily state or reflect those of the United States Government or any agency thereof. 


\section{Table of Contents}

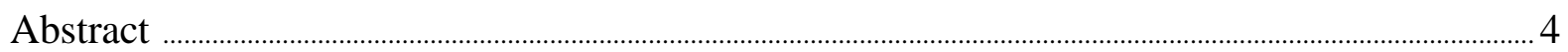

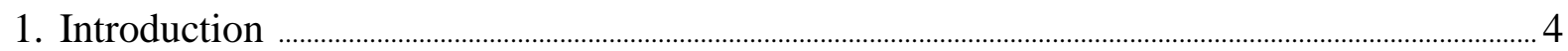

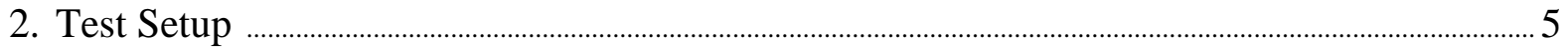

a. Description of Test Refrigerant, Lubricant, and Charge....................................................................... 5

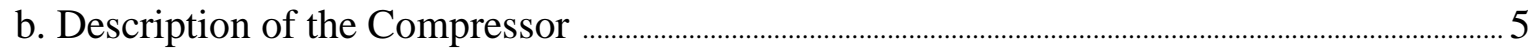

c. Description of the Compressor Calorimeter Test Loop …………........................................................... 6

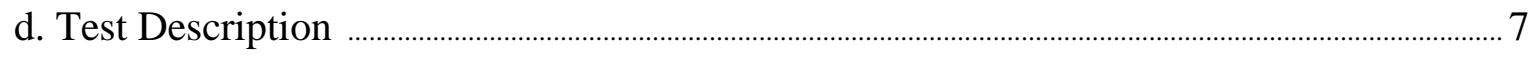

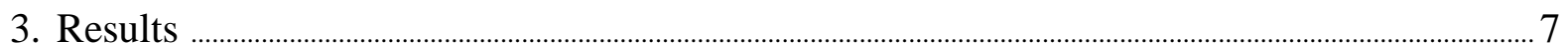

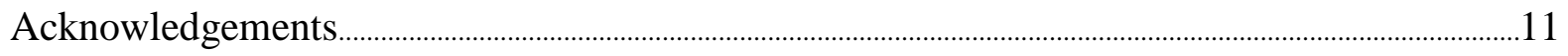

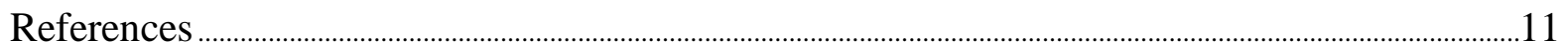

Appendix A: Tabular Data ..........................................................................................................................................12

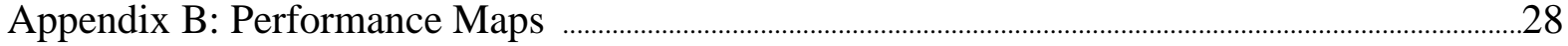

Capacity, Input Power, and EER …....................................................................................................28

10-Coefficient polynomial equation for each test refrigerant .....................................................38 


\section{Abstract}

As a contribution to the AHRI Low-GWP Alternative Refrigerants Evaluation Program (AREP), this study compares the performance of four lower-GWP alternative refrigerants, ARM-31a, D2Y-65, L-40, and R-32 + R-134a mixture, to that of refrigerant $\mathrm{R}-404 \mathrm{~A}$ (baseline) in a scroll compressor designed for medium temperature refrigeration applications. These comparisons were carried out via compressor calorimeter tests performed on a compressor designed for refrigerant R-404A and having a nominal rated capacity of 23,500 Btu/hr.

Tests were conducted over a suction dew point temperature range of $-10^{\circ} \mathrm{F}$ to $35^{\circ} \mathrm{F}$ in $5^{\circ} \mathrm{F}$ increments and a discharge dew point temperature range of $70^{\circ} \mathrm{F}$ to $140^{\circ} \mathrm{F}$ in $10^{\circ} \mathrm{F}$ increments. All the tests were performed with $20^{\circ} \mathrm{F}$ superheat, $40^{\circ} \mathrm{F}$ superheat, and $65^{\circ} \mathrm{F}$ suction temperature. A liquid subcooling level of $10^{\circ} \mathrm{F}$ to $15^{\circ} \mathrm{F}$ was maintained for all the test conditions. However, the cooling capacities reported in this study are normalized for $0^{\circ} \mathrm{F}$ subcooling.

The tests showed that the compressor energy efficiency ratio (EER) and cooling capacity with all four alternative refrigerants tested are higher at higher saturation suction and saturation discharge temperature and lower at lower saturation suction and saturation discharge temperature, compared to that of R-404A. Discharge temperatures of all the alternative refrigerants were higher than that of R-404A at all test conditions.

\section{Introduction}

This report investigates the tested performance of lower-GWP candidate refrigerants ARM-31a, D2Y-65, L-40, and a 50.53\% R-32 + 49.47\% R-134a mixture (hereafter referred to as R-32 + R-134a mixture) as alternatives for the baseline refrigerant R-404A using a 36,000 Btu/hr compressor calorimeter located at the Heat Exchanger Advanced Testing Facility in building 5800 at Oak Ridge National Laboratory. These tests were conducted during January to March 2013.

$\mathrm{R}-404 \mathrm{~A}$ is a near-azeotropic blend of R125, R-143a, and R-134a with 0.44/0.52/0.04 mass fraction and has a GWP of $3260^{1}$. ARM-31a, D2Y-65, L-40, and a R-32 + R-134a mixture are new blends with lower GWP and are under development by Arkema, Daikin, Honeywell, and National Refrigerants, respectively.

This report compares various performance parameters, such as cooling capacity, compressor power, refrigerant mass flow rate, EER, isentropic efficiency and discharge temperature, of four alternative refrigerants to that of R-404A.

1 Source: "2010 Report of the refrigeration, air conditioning and heat pumps technical options committee; Chapter 2, Refrigerants." United Nations Environment Programme (UNEP) Ozone Secretariat, Nairobi, Kenya, http://ozone.unep.org/teap/Reports/RTOC/RTOC-Assessment-report2010.pdf. 


\section{Test Setup}

\section{a. Description of Test Refrigerant, Lubricant, and Charge}

- Refrigerant or refrigerant blend tested

o R-404A: R125, R-143a, and R-134a blend with 0.44/0.52/0.04 mass fraction

o ARM-31a

o D2Y-65

o L-40

o R-32 + R-134a mixture with 0.5053/0. 4947 mass fraction

- Refrigerant charge: The refrigerant charge required to achieve similar subcooling after the condenser at identical test conditions is given as follows:

o R-404A: 3 lbs 12 ounces

o ARM-31a: 3 lbs 12 ounces

o D2Y-65: 3 lbs 8 ounces

o L-40: 4 lbs

o R-32 + R-134a mixture: 3 lb 12 ounces

- Lubricant

o The lubricant used for all the tests is the original lubricant charged in the scroll compressor used for the testing. As per the manufacturer's data sheet, the compressor was charged with $42 \mathrm{oz}$ of polyolester oil (POE).

\section{b. Description of the Compressor}

The compressor used for this test was a Copeland hermetic scroll compressor ZS21KAEPFV that has a 7/8" suction port and a 1/2" discharge port. This hermetic compressor uses a 208/230 volt, single phase, $60 \mathrm{~Hz}$ electric motor. The displacement volume of the compressor is $3.11 \mathrm{in}^{3} / \mathrm{rev}$ or $377.71 \mathrm{ft}^{3} / \mathrm{hr}$. Table 1 shows the manufacturer-provided performance of the compressor at standard test conditions.

Table 1 Manufacturer's Compressor Performance Data

\begin{tabular}{|lc|}
\hline Evaporating $\left({ }^{\circ} \mathrm{F}\right) /$ Condensing $\left({ }^{\circ} \mathrm{F}\right)$ & $20 / 120$ \\
Return Gas $\left({ }^{\circ} \mathrm{F}\right) /$ Liquid to & $65 / 120$ \\
\hline Expansion Valve $\left({ }^{\circ} \mathrm{F}\right)$ & 23500 \\
\hline Capacity (Btu/hr) & 3320 \\
Power (Watts) & 15.60 \\
Current (Amps) & 7.10 \\
EER (Btu/Wh) & 495 \\
Mass Flow (lbs/hr) & \\
\hline
\end{tabular}


No modification to the compressor or the lubricant was made for the tests documented in this report. The compressor was uninsulated and airflow to the compressor chamber was maintained by two small fans, circulating air from outlets about $1.5 \mathrm{ft}$ above the compressor. Combined airflow rate of the two fans was $750 \mathrm{cfm}$. The compressor chamber air temperature was maintained at $95 \pm 1^{\circ} \mathrm{F}$ for all the tests.

To assure the accuracy of the test set-up, data acquisition hardware, and the software, performance of the test compressor calculated from the baseline test was compared with the compressor map provided by the compressor manufacturer. This confirmed that the test data are in good agreement with compressor manufacturer provided data. At standard test conditions (saturation suction temperature of $20^{\circ} \mathrm{F}$, suction temperature of $65^{\circ} \mathrm{F}$, saturation discharge temperature of $120^{\circ} \mathrm{F}$, and liquid temperature of $120^{\circ} \mathrm{F}$ ), the calculated EER and capacity from the test data were within $\pm 0.2 \%$ and $\pm 2.7 \%$, respectively, of the compressor manufacturer's data. This is well within the $\pm 5 \%$ tolerance allowed in ANSI/AHRI 540-2004 for capacity and efficiency.

\section{c. Description of the Compressor Calorimeter Test Loop}

The compressor calorimeter test loop, shown in Figure 1, consists of a test compressor, a condenser, a sub-cooler, three electronic expansion valves, and an evaporator. Suction pressure, suction temperature, liquid temperature, compressor chamber air temperature, and discharge pressure are controlled independently by controlling the electronic expansion valve, evaporator heater output, secondary glycol temperature, heating or cooling the air within the compressor chamber, and temperature of the condenser, respectively. Figure 1 also shows the location of the various sensors in the compressor calorimeter test loop. Table 2 lists the accuracy of the test instruments used in the calorimeter.

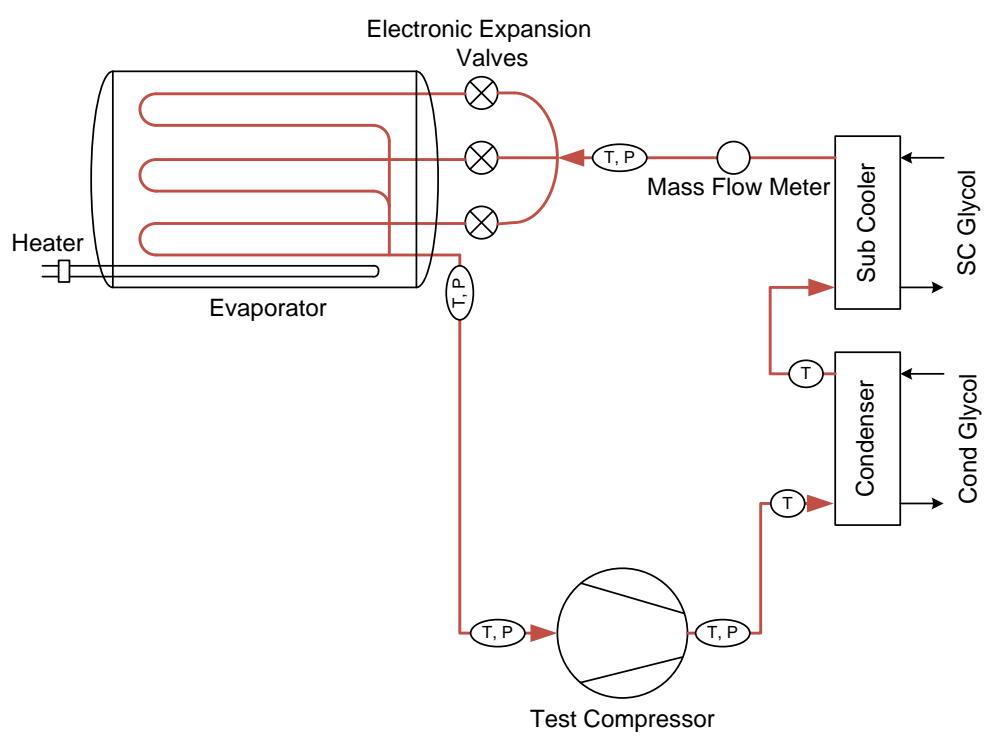

Figure 1 Location of various sensors in the compressor calorimeter test loop. 
Table 2. Accuracy of the Instruments used in the Compressor Calorimeter Test Loop

\begin{tabular}{|l|l|c|c|}
\hline Instrument & Measured Parameter & $\begin{array}{c}\text { Measurement } \\
\text { Range }\end{array}$ & Accuracy \\
\hline \hline RTDs & Temperature & $\pm 0.4^{\circ} \mathrm{F}$ \\
\hline $\begin{array}{l}\text { Micro Motion Elite } \\
\text { Sensor }\end{array}$ & $\begin{array}{l}\text { Refrigerant mass flow } \\
\text { rate }\end{array}$ & $\pm 0.10 \%$ \\
\hline $\begin{array}{l}\text { Yokogawa Power and } \\
\text { Energy Meter PR300 }\end{array}$ & Compressor power & $\pm 0.5 \%$ \\
\hline $\begin{array}{l}\text { Honeywell Pressure } \\
\text { Transmitter 060-F444- } \\
\text { 02 }\end{array}$ & $\begin{array}{l}\text { Refrigerant pressure, } \\
\text { high pressure side }\end{array}$ & 750 psia & $0.25 \%$ F.S. \\
\hline $\begin{array}{l}\text { Honeywell Pressure } \\
\text { Transmitter 060-F443- } \\
\text { 05 }\end{array}$ & $\begin{array}{l}\text { Refrigerant pressure, } \\
\text { low pressure side }\end{array}$ & 200 psia & $0.25 \%$ F.S. \\
\hline
\end{tabular}

\section{d. Test Description}

The compressor calorimetry was performed according to ANSI/ASHRAE Standard 23.12010. Before testing each alternative refrigerant, performance of the test compressor was checked using the baseline refrigerant R-404A. The intermediate tests confirmed repeatability of cooling capacity within $0.45 \%$ and EER within $0.50 \%$ at the standard test condition.

Tests were conducted over a wide range of operating conditions. The suction dew point temperature was varied between $-10^{\circ} \mathrm{F}$ and $35^{\circ} \mathrm{F}$ in $5^{\circ} \mathrm{F}$ increments while the discharge dew point temperature was varied between $70^{\circ} \mathrm{F}$ and $140^{\circ} \mathrm{F}$ in $10^{\circ} \mathrm{F}$ increments. These tests were performed with $10^{\circ} \mathrm{F}$ to $15^{\circ} \mathrm{F}$ subcooling and either $20^{\circ} \mathrm{F}$ or $40^{\circ} \mathrm{F}$ superheat. Tests were also conducted at a fixed $65^{\circ} \mathrm{F}$ suction temperature. The cooling capacities reported in this report are normalized for $0^{\circ} \mathrm{F}$ subcooling.

For compressor safety, the maximum discharge temperature was set at $275^{\circ} \mathrm{F}$ for all tests. Therefore refrigerants with higher discharge temperature yielded lower number of test data points compared to the refrigerants with lower discharge temperature. Test conditions with lower suction dew point temperature and higher discharge dew point temperature combinations were dropped due to the limit on discharge temperature.

\section{Results}

Appendix A provides summary results of all the tests conducted for this study in a tabular form. Appendix B provides 10-coefficient polynomial equations for mass flow, capacity, power, EER and discharge temperature computed from the test results for each test refrigerant at standard rating conditions of $65^{\circ} \mathrm{F}$ suction temperature and $0^{\circ} \mathrm{F}$ subcooling. Appendix B also presents figures showing capacity, input power, EER, and isentropic efficiency as a function of suction dew point temperature for given discharge dew point 
temperature at $65^{\circ} \mathrm{F}$ suction temperature and $0^{\circ} \mathrm{F}$ subcooling for $\mathrm{R}-404 \mathrm{~A}$ and each alternative refrigerant tested. The isentropic efficiency plots show the trends and shifts in compressor performance separately from the refrigerant property performance.

The properties of R-404A were calculated using REFPROP version 9.0 (Lemmon et al, 2010). Interaction parameters and "mixture" files provided by the refrigerant manufacturers were used in REFPROP to calculate properties of the alternative refrigerants tested.

Table 3 shows differences in EER, capacity, and discharge temperature of the four alternative refrigerants compared to that of R-404A at $0^{\circ} \mathrm{F}$ subcooling, $65^{\circ} \mathrm{F}$ suction temperature, $70^{\circ} \mathrm{F}$ to $140^{\circ} \mathrm{F}$ saturation discharge temperature and $-10^{\circ} \mathrm{F}$ to $35^{\circ} \mathrm{F}$ saturation suction temperature. The table also shows the change in performance parameters at the standard test condition of $120^{\circ} \mathrm{F}$ discharge dew point temperature and $20^{\circ} \mathrm{F}$ suction dew point temperature.

Table 3. Performance of Alternative Refrigerants compared to that of R-404A

\begin{tabular}{|c|c|c|c|c|}
\hline \multirow[b]{2}{*}{ Refrigerant } & \multirow[b]{2}{*}{ Test Condition } & \multicolumn{3}{|c|}{ Deviation from baseline (R-404A) } \\
\hline & & $\operatorname{EER}(\%)$ & Capacity (\%) & $\begin{array}{c}\text { Discharge } \\
\text { Temperature }\left({ }^{\circ} \mathrm{R}\right)\end{array}$ \\
\hline \multirow{3}{*}{ ARM-31a } & Evap $20^{\circ} \mathrm{F} /$ Cond $120^{\circ} \mathrm{F}$ & $+9.7 \%$ & $-5.6 \%$ & +24 \\
\hline & Over the range & $-4.4 \%$ to $+22.8 \%$ & $-18.5 \%$ to $+6.5 \%$ & +16 to +36 \\
\hline & Average & $+6.2 \%$ & $-9.1 \%$ & +25 \\
\hline \multirow{3}{*}{ D2Y-65 } & Evap $20^{\circ} \mathrm{F} /$ Cond $120^{\circ} \mathrm{F}$ & $+6.5 \%$ & $+0.6 \%$ & +26 \\
\hline & Over the range & $-4.2 \%$ to $+14.6 \%$ & $-11.0 \%$ to $+9.7 \%$ & +18 to +36 \\
\hline & Average & $+3.2 \%$ & $-2.9 \%$ & +27 \\
\hline \multirow{3}{*}{ L-40 } & Evap $20^{\circ} \mathrm{F} /$ Cond $120^{\circ} \mathrm{F}$ & $+9.8 \%$ & $-4.9 \%$ & +37 \\
\hline & Over the range & $-5.4 \%$ to $+24.7 \%$ & $-20.0 \%$ to $+8.9 \%$ & +25 to +53 \\
\hline & Average & $+5.3 \%$ & $-9.7 \%$ & +37 \\
\hline \multirow{3}{*}{$\begin{array}{l}\text { R-32+ } \\
\text { R-134a } \\
\text { mixture }\end{array}$} & Evap $20^{\circ} \mathrm{F} /$ Cond $120^{\circ} \mathrm{F}$ & $+4.2 \%$ & $+3.8 \%$ & +63 \\
\hline & Over the range & $-7.6 \%$ to $+15.2 \%$ & $-12.6 \%$ to $+18 \%$ & +38 to +74 \\
\hline & Average & $+1.0 \%$ & $-2.1 \%$ & +54 \\
\hline
\end{tabular}

Figures 2 to 5 present the ratios of $\mathrm{EER}_{\text {alt }}$ and $\mathrm{EER}_{\text {Baseline }}$ as a function of suction dew point temperature for given discharge dew point temperature at $65^{\circ} \mathrm{F}$ suction temperature and $0^{\circ} \mathrm{F}$ subcooling for each alternative refrigerant tested. At almost all suction dew point temperatures used in these tests the $\mathrm{EER}_{\mathrm{alt}} / \mathrm{EER}_{\text {Baseline was greater at higher discharge dew }}$ point temperature (110 to $110^{\circ} \mathrm{F}$ or higher) for ARM-31a, D2Y-64, and L-40; however, for the R-32 + R-134a mixture this was true only for suction dew point temperatupe $15^{\circ} \mathrm{F}$. 


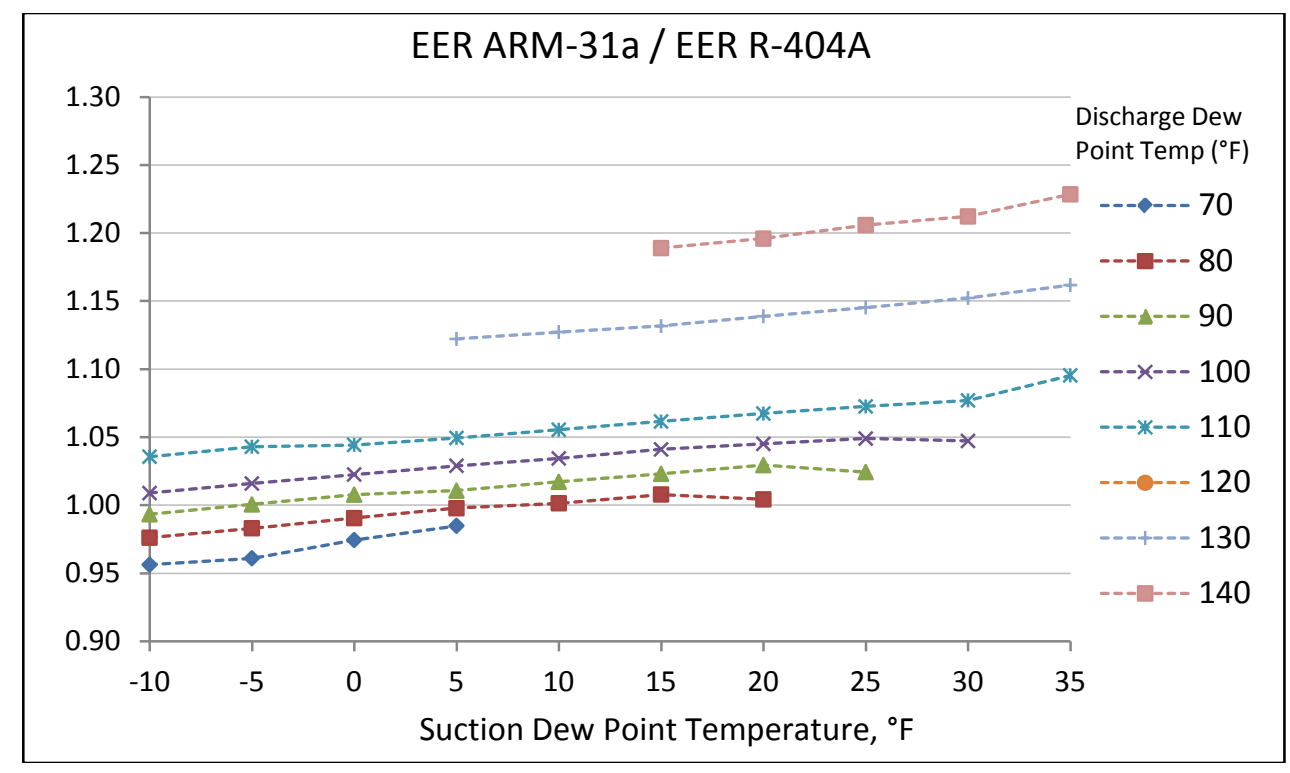

Figure 2 EER of ARM-31a compared to EER of R-404A.

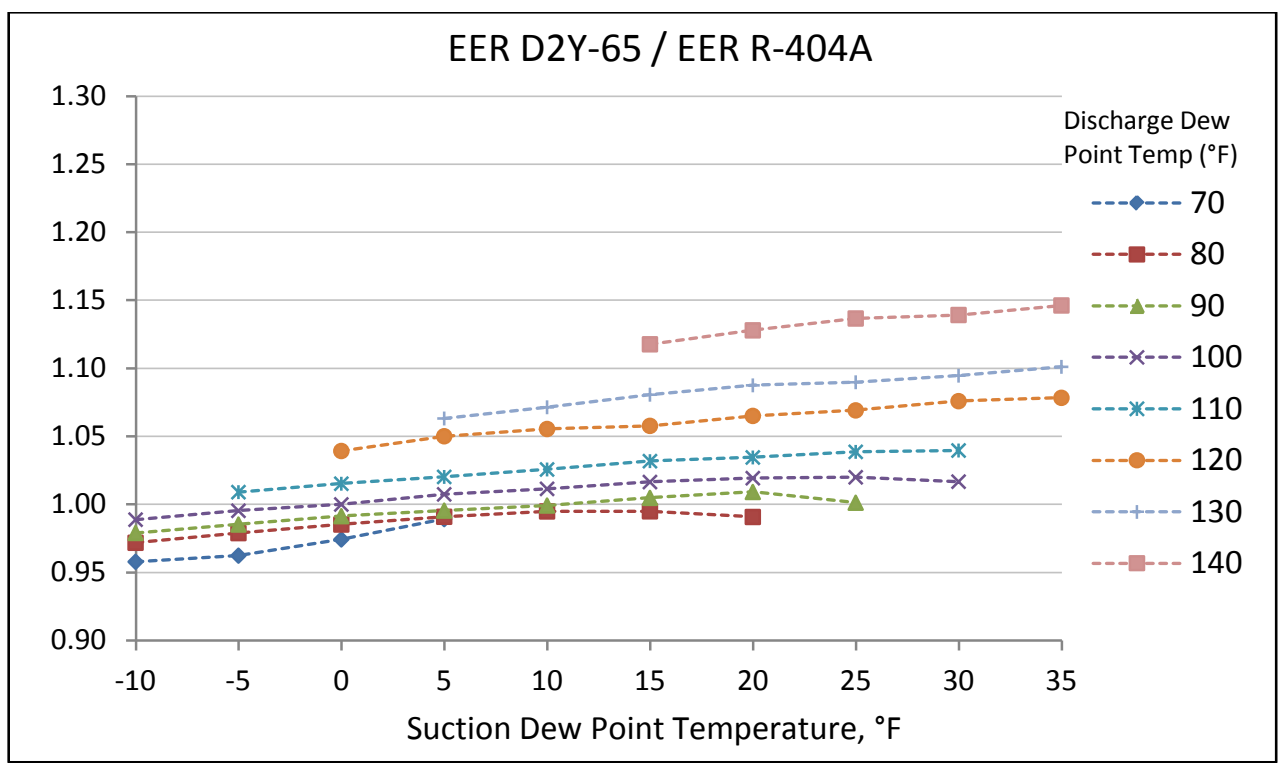

Figure 3 EER of D2Y-65 compared to EER of R-404A. 


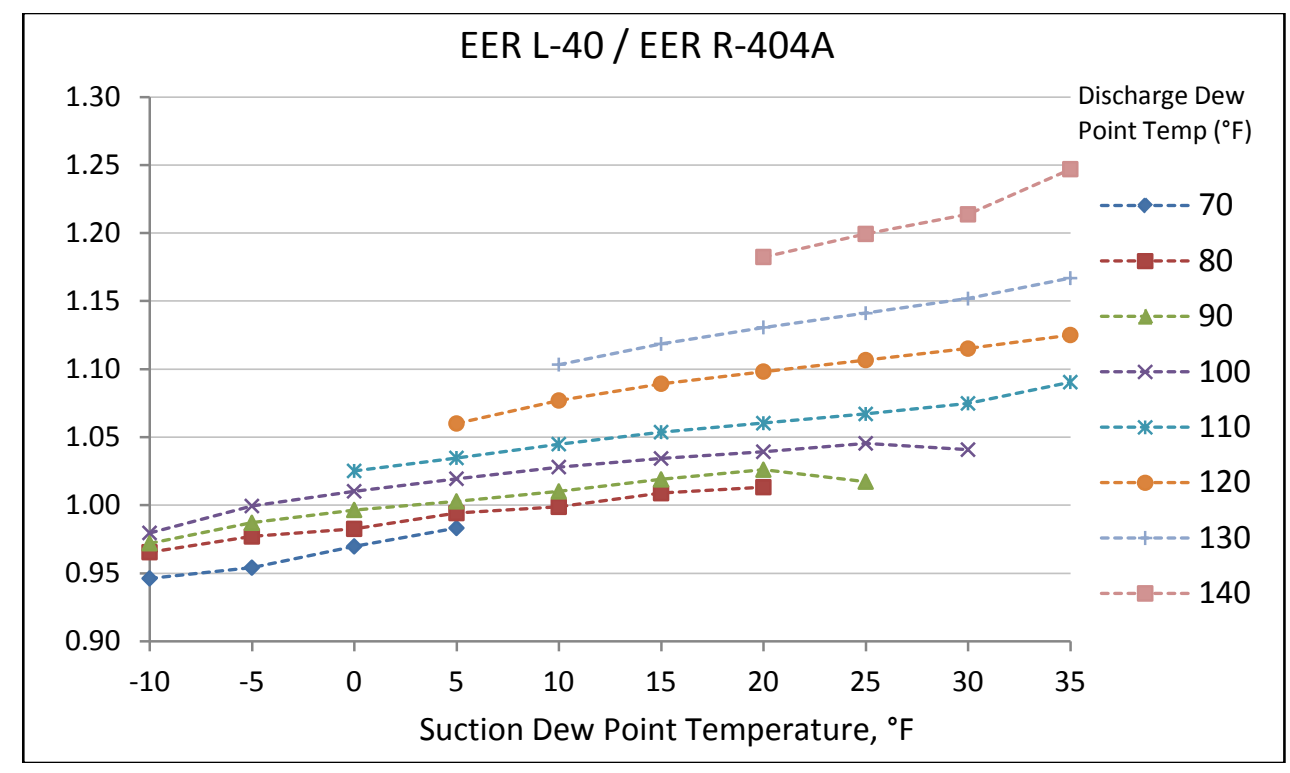

Figure 4 EER of L-40 compared to EER of R-404A.

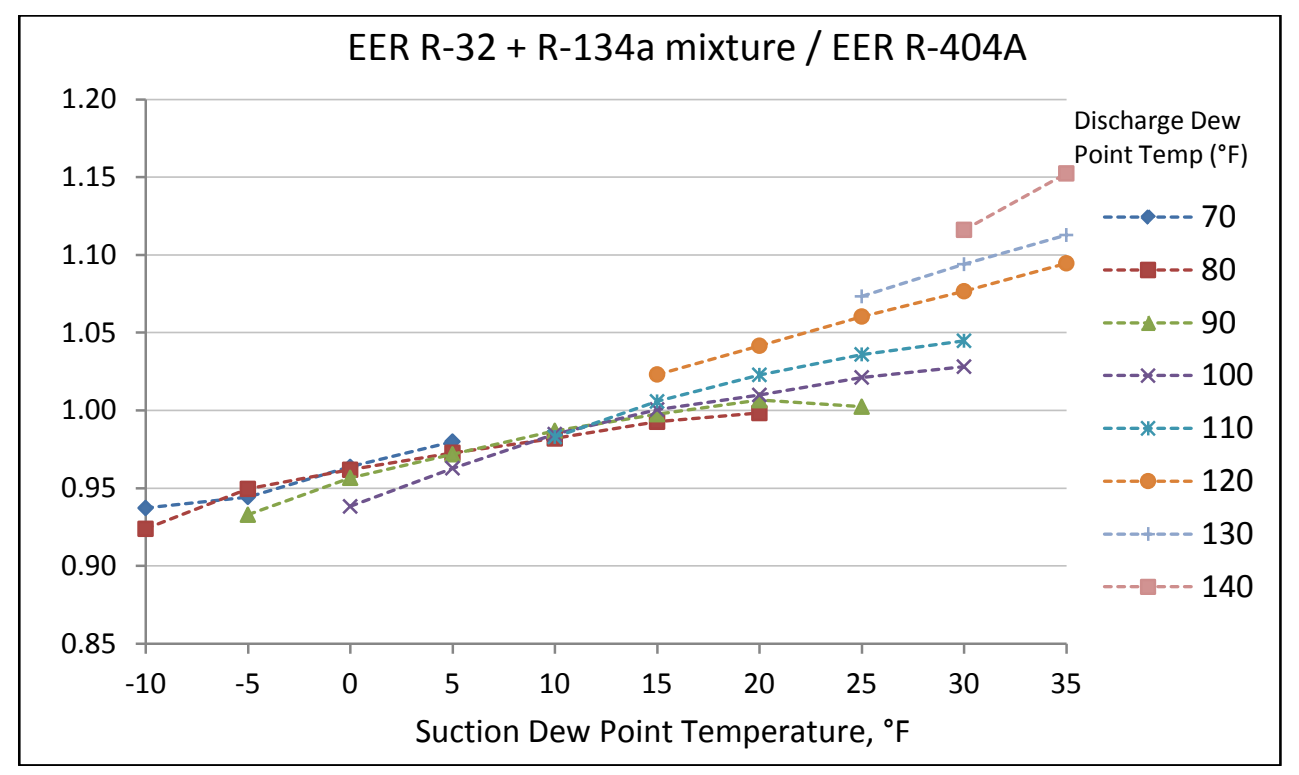

Figure 5 EER of R-32 + R-134a mixture compared to EER of R-404A. 


\section{Acknowledgements}

This work was supported by the Building Technologies Program, U.S. Department of Energy. The authors would like to acknowledge the contributions of Brian Goins and Randall Linkous for their technical support.

\section{References}

Lemmon, E.W. Huber, M.L., and McLinden, M. O., 2010, NIST Standard Reference Database 23, NIST Reference Fluid Thermodynamic and Transport Properties-REFPROP. Version 9.0 User's Guide, November 2010.

ANSI/AHRI 540-2004, Standard for Performance Rating of Positive Displacement Refrigerant Compressors and Compressor Units

ANSI/ASHRAE Standard 23.1-2010, Methods of Testing for Rating the Performance of Positive Displacement Refrigerant Compressors and Condensing Units that Operate at Subcritical Temperatures of the Refrigerant 


\section{Appendix A}

\section{$\underline{\text { Tabular Data }}$}

Tables which follow provide summary data of all tests conducted for this study. Applicable superheat and subcooling levels for each test are provided in each table heading. Performance parameters such as cooling capacity, compressor power, compressor current, refrigerant mass flow rate, EER, isentropic efficiency, and discharge temperature are tabulated as a function of discharge dew point temperature and suction dew point temperature for fixed degree of superheat or fixed suction temperature. Since EER values are provided, COP values are not included in these tables. 
Table A1: R-404A at $65^{\circ} \mathrm{F}$ Suction Temperature, $0^{\circ} \mathrm{F}$ Subcooling

Suction Dew Point Temp, ${ }^{\circ} \mathrm{F}$ (Suction Pressure, psia)

\begin{tabular}{|c|c|c|c|c|c|c|c|c|c|c|c|}
\hline & & $-10(38)$ & $-5(42)$ & $0(47)$ & $5(52)$ & $10(57)$ & $15(63)$ & $20(69)$ & $25(76)$ & $30(84)$ & 35 (91) \\
\hline \multirow{7}{*}{$\begin{array}{c}140 \\
(416)\end{array}$} & C & & & & 13348 & 14815 & 16572 & 18338 & 20230 & 22247 & 24222 \\
\hline & $P$ & & & & 3837 & 3889 & 3952 & 3998 & 4052 & 4104 & 4168 \\
\hline & A & & & & 17.65 & 17.87 & 18.16 & 18.36 & 18.60 & 18.83 & 19.11 \\
\hline & $M$ & & & & 338.1 & 379.2 & 425.7 & 475.2 & 529.3 & 588.8 & 651.4 \\
\hline & $E$ & & & & 3.48 & 3.81 & 4.19 & 4.59 & 4.99 & 5.42 & 5.81 \\
\hline & $\%$ & & & & 62.4 & 64.2 & 66.2 & 67.9 & 69.3 & 70.5 & 70.9 \\
\hline & $\mathrm{T}$ & & & & 251.5 & 240.6 & 229.3 & 219.6 & 210.9 & 202.9 & 196.5 \\
\hline \multirow{7}{*}{$\begin{array}{c}130 \\
(368)\end{array}$} & C & & 12088 & 13630 & 15255 & 17012 & 18874 & 20879 & 23070 & 25377 & 27743 \\
\hline & $P$ & & 3388 & 3403 & 3487 & 3523 & 3566 & 3617 & 3669 & 3714 & 3765 \\
\hline & A & & 15.73 & 15.80 & 16.16 & 16.32 & 16.52 & 16.73 & 16.96 & 17.17 & 17.38 \\
\hline & $M$ & & 270.3 & 306.1 & 344.1 & 386.2 & 431.4 & 480.9 & 536.2 & 595.5 & 660.0 \\
\hline & $E$ & & 3.57 & 4.01 & 4.38 & 4.83 & 5.29 & 5.77 & 6.29 & 6.83 & 7.37 \\
\hline & $\%$ & & 61.4 & 63.3 & 65.5 & 67.5 & 69.2 & 70.6 & 71.6 & 72.7 & 72.8 \\
\hline & $\mathrm{T}$ & & 259.2 & 246.2 & 234.2 & 223.3 & 213.5 & 204.8 & 196.8 & 189.3 & 183.0 \\
\hline \multirow{7}{*}{$\begin{array}{c}120 \\
(324)\end{array}$} & C & 12003 & 13509 & 15165 & 16937 & 18862 & 20945 & 23192 & 25611 & 28216 & 30992 \\
\hline & $P$ & 2955 & 3009 & 3063 & 3121 & 3175 & 3229 & 3278 & 3322 & 3364 & 3397 \\
\hline & $A$ & 13.94 & 14.17 & 14.40 & 14.64 & 14.87 & 15.10 & 15.31 & 15.50 & 15.67 & 15.82 \\
\hline & $M$ & 242.5 & 274.2 & 309.2 & 347.1 & 388.7 & 434.6 & 484.5 & 539.5 & 600.1 & 666.3 \\
\hline & $E$ & 4.06 & 4.49 & 4.95 & 5.43 & 5.94 & 6.49 & 7.07 & 7.71 & 8.39 & 9.12 \\
\hline & $\%$ & 63.8 & 65.6 & 67.4 & 68.8 & 70.1 & 71.2 & 72.2 & 73.2 & 73.7 & 74.2 \\
\hline & $\mathrm{T}$ & 251.0 & 239.0 & 227.6 & 217.5 & 208.2 & 199.5 & 191.4 & 183.8 & 176.8 & 170.1 \\
\hline \multirow{7}{*}{$\begin{array}{c}110 \\
(285)\end{array}$} & C & 13267 & 14942 & 16737 & 18687 & 20783 & 23084 & 25585 & 28285 & 31194 & 33875 \\
\hline & $P$ & 2738 & 2771 & 2804 & 2846 & 2892 & 2938 & 2977 & 3009 & 3035 & 3059 \\
\hline & $A$ & 13.06 & 13.20 & 13.33 & 13.50 & 13.70 & 13.90 & 14.05 & 14.19 & 14.30 & 14.41 \\
\hline & $M$ & 247.1 & 279.4 & 314.4 & 352.7 & 394.3 & 440.7 & 491.7 & 547.5 & 608.5 & 667.8 \\
\hline & $E$ & 4.85 & 5.39 & 5.97 & 6.57 & 7.19 & 7.86 & 8.60 & 9.40 & 10.28 & 11.07 \\
\hline & $\%$ & 65.6 & 67.6 & 69.3 & 70.6 & 71.9 & 72.7 & 73.5 & 74.2 & 74.9 & 74.7 \\
\hline & $\mathrm{T}$ & 235.7 & 223.6 & 212.9 & 203.4 & 194.4 & 186.3 & 178.5 & 171.2 & 164.1 & 158.7 \\
\hline \multirow{7}{*}{$\begin{array}{c}100 \\
(249)\end{array}$} & $C$ & 14473 & 16241 & 18170 & 20270 & 22557 & 25052 & 27769 & 30702 & 33975 & \\
\hline & $P$ & 2469 & 2508 & 2548 & 2591 & 2630 & 2665 & 2692 & 2714 & 2734 & \\
\hline & $A$ & 11.98 & 12.15 & 12.31 & 12.49 & 12.64 & 12.79 & 12.90 & 12.99 & 13.07 & \\
\hline & $M$ & 250.9 & 282.5 & 317.4 & 355.7 & 397.8 & 444.2 & 495.3 & 551.3 & 615.4 & \\
\hline & $E$ & 5.86 & 6.48 & 7.13 & 7.82 & 8.58 & 9.40 & 10.31 & 11.31 & 12.43 & \\
\hline & $\%$ & 68.3 & 69.8 & 70.9 & 71.8 & 72.6 & 73.4 & 73.9 & 74.5 & 74.7 & \\
\hline & $\mathrm{T}$ & 219.0 & 208.4 & 199.0 & 190.2 & 181.9 & 174.0 & 166.6 & 159.3 & 152.4 & \\
\hline \multirow{7}{*}{$\begin{array}{c}90 \\
(217)\end{array}$} & C & 15569 & 17462 & 19509 & 21827 & 24256 & 26938 & 29836 & 33095 & & \\
\hline & $P$ & 2242 & 2282 & 2318 & 2353 & 2383 & 2410 & 2431 & 2441 & & \\
\hline & A & 11.11 & 11.26 & 11.40 & 11.54 & 11.66 & 11.77 & 11.86 & 11.90 & & \\
\hline & $M$ & 252.9 & 284.7 & 319.3 & 358.8 & 400.4 & 447.0 & 498.4 & 556.0 & & \\
\hline & $E$ & 6.94 & 7.65 & 8.42 & 9.27 & 10.18 & 11.18 & 12.27 & 13.56 & & \\
\hline & $\%$ & 70.0 & 70.9 & 71.7 & 72.6 & 72.8 & 73.1 & 73.3 & 73.6 & & \\
\hline & $\mathrm{T}$ & 204.3 & 195.0 & 186.3 & 177.5 & 169.9 & 162.5 & 155.3 & 148.0 & & \\
\hline \multirow{7}{*}{$\begin{array}{c}80 \\
(188)\end{array}$} & C & 16627 & 18625 & 20842 & 23245 & 25906 & 28735 & 31911 & & & \\
\hline & P & 2043 & 2077 & 2108 & 2136 & 2157 & 2174 & 2180 & & & \\
\hline & A & 10.34 & 10.47 & 10.58 & 10.70 & 10.78 & 10.84 & 10.86 & & & \\
\hline & $M$ & 254.6 & 286.1 & 321.4 & 359.8 & 402.7 & 449.1 & 501.2 & & & \\
\hline & $\mathrm{E}$ & 8.14 & 8.97 & 9.89 & 10.88 & 12.01 & 13.22 & 14.64 & & & \\
\hline & $\%$ & 70.8 & 71.3 & 71.5 & 71.7 & 72.0 & 72.0 & 71.2 & & & \\
\hline & $\mathrm{T}$ & 191.1 & 182.5 & 174.3 & 166.4 & 158.6 & 151.5 & 144.4 & & & \\
\hline \multirow{7}{*}{$\begin{array}{c}70 \\
(162)\end{array}$} & C & 17722 & 19885 & 22142 & 24628 & & & & & & \\
\hline & $P$ & 1856 & 1881 & 1910 & 1931 & & & & & & \\
\hline & $A$ & 9.62 & 9.73 & 9.84 & 9.92 & & & & & & \\
\hline & $M$ & 257.1 & 289.1 & 323.5 & 361.4 & & & & & & \\
\hline & $\mathrm{E}$ & 9.55 & 10.57 & 11.59 & 12.75 & & & & & & \\
\hline & $\%$ & 70.6 & 70.4 & 70.4 & 70.4 & & & & & & \\
\hline & $\mathrm{T}$ & 179.0 & 170.7 & 163.3 & 155.8 & & & & & & \\
\hline
\end{tabular}

C: Capacity (Btu/hr), P: Power (Watts), A: Current (Amps) at 230V, M: Mass Flow (lbs/hr), E: EER (Btu/Watt-hr) $\%$ : Isentropic Efficiency (\%), T: Discharge Temperature $\left({ }^{\circ} \mathrm{F}\right)$ 
Table A2: R-404A at $20^{\circ} \mathrm{F}$ Superheat, $0^{\circ} \mathrm{F}$ Subcooling

Suction Dew Point Temp, ${ }^{\circ} \mathrm{F}$ (Suction Pressure, psia)

\begin{tabular}{|c|c|c|c|c|c|c|c|c|c|c|c|}
\hline & & $-10(38)$ & $-5(42)$ & $0(47)$ & $5(52)$. & 10 (57.) & 15 (63.) & 20 (69.) & 25 (76.) & 30 (84.) & 35 (91.) \\
\hline \multirow{7}{*}{$\begin{array}{c}140 \\
(416)\end{array}$} & C & & & & 11444 & 12941 & 14756 & 16667 & 18742 & 21006 & 23458 \\
\hline & $\mathrm{P}$ & & & & 3804 & 3873 & 3930 & 3985 & 4043 & 4096 & 4159 \\
\hline & $A$ & & & & 17.54 & 17.83 & 18.09 & 18.31 & 18.57 & 18.81 & 19.08 \\
\hline & $M$ & & & & 372.1 & 412.6 & 458.1 & 506.6 & 557.8 & 612.9 & 672.7 \\
\hline & $E$ & & & & 3.01 & 3.34 & 3.75 & 4.18 & 4.64 & 5.13 & 5.64 \\
\hline & $\%$ & & & & 58.8 & 61.0 & 62.8 & 64.9 & 66.8 & 68.6 & 70.2 \\
\hline & $\mathrm{T}$ & & & & 214.5 & 208.8 & 202.7 & 197.7 & 193.5 & 190.0 & 187.2 \\
\hline \multirow{7}{*}{$\begin{array}{c}130 \\
(368)\end{array}$} & $C$ & & 10290 & 11795 & 13437 & 15217 & 17179 & 19348 & 21706 & 24280 & 26885 \\
\hline & $P$ & & 3338 & 3385 & 3454 & 3509 & 3560 & 3611 & 3662 & 3713 & 3757 \\
\hline & A & & 15.57 & 15.76 & 16.06 & 16.28 & 16.51 & 16.73 & 16.95 & 17.16 & 17.36 \\
\hline & $M$ & & 301.9 & 337.9 & 377.4 & 419.2 & 463.9 & 512.6 & 565.2 & 621.2 & 677.4 \\
\hline & $E$ & & 3.08 & 3.48 & 3.89 & 4.34 & 4.83 & 5.36 & 5.93 & 6.54 & 7.16 \\
\hline & $\%$ & & 56.9 & 59.0 & 61.4 & 63.4 & 65.4 & 67.3 & 69.1 & 70.7 & 72.3 \\
\hline & $\mathrm{T}$ & & 213.1 & 205.6 & 198.0 & 192.4 & 187.3 & 183.0 & 179.3 & 176.2 & 174.1 \\
\hline \multirow{7}{*}{$\begin{array}{c}120 \\
(324)\end{array}$} & C & 10339 & 11843 & 13494 & 15287 & 17287 & 19464 & 21847 & 24443 & 27335 & 30046 \\
\hline & $P$ & 2962 & 3022 & 3088 & 3137 & 3191 & 3238 & 3282 & 3321 & 3360 & 3396 \\
\hline & A & 14.00 & 14.25 & 14.52 & 14.73 & 14.96 & 15.15 & 15.34 & 15.51 & 15.67 & 15.82 \\
\hline & $M$ & 272.8 & 306.4 & 343.2 & 381.8 & 424.0 & 469.1 & 518.1 & 570.4 & 627.8 & 679.9 \\
\hline & $E$ & 3.49 & 3.92 & 4.37 & 4.87 & 5.42 & 6.01 & 6.66 & 7.36 & 8.13 & 8.85 \\
\hline & $\%$ & 56.3 & 59.0 & 61.6 & 63.4 & 65.3 & 67.1 & 68.9 & 70.7 & 72.3 & 74.0 \\
\hline & $T$ & 205.3 & 196.6 & 188.5 & 183.0 & 177.7 & 173.4 & 169.5 & 166.0 & 163.0 & 161.5 \\
\hline \multirow{7}{*}{$\begin{array}{c}110 \\
(285)\end{array}$} & $C$ & 11656 & 13273 & 15087 & 17061 & 19248 & 21613 & 24250 & 27133 & 30342 & \\
\hline & $P$ & 2715 & 2767 & 2815 & 2863 & 2907 & 2946 & 2982 & 3012 & 3035 & \\
\hline & A & 12.99 & 13.21 & 13.40 & 13.60 & 13.78 & 13.94 & 14.09 & 14.21 & 14.31 & \\
\hline & $M$ & 277.3 & 310.3 & 346.7 & 385.6 & 428.0 & 473.1 & 523.1 & 576.5 & 635.6 & \\
\hline & $E$ & 4.29 & 4.80 & 5.36 & 5.96 & 6.62 & 7.34 & 8.13 & 9.01 & 10.00 & \\
\hline & $\%$ & 59.0 & 61.0 & 63.1 & 64.9 & 66.7 & 68.4 & 70.1 & 71.7 & 73.2 & \\
\hline & $\mathrm{T}$ & 187.5 & 180.4 & 174.1 & 168.9 & 164.3 & 160.1 & 156.5 & 153.3 & 150.5 & \\
\hline \multirow{7}{*}{$\begin{array}{c}100 \\
(249)\end{array}$} & C & 12893 & 14659 & 16639 & 18781 & 21152 & 23737 & 26555 & 29698 & 32835 & \\
\hline & $P$ & 2482 & 2531 & 2574 & 2612 & 2647 & 2679 & 2704 & 2722 & 2740 & \\
\hline & $A$ & 12.06 & 12.26 & 12.42 & 12.58 & 12.72 & 12.85 & 12.96 & 13.03 & 13.10 & \\
\hline & $M$ & 280.0 & 313.3 & 350.1 & 389.2 & 431.9 & 477.8 & 526.9 & 581.5 & 635.5 & \\
\hline & $E$ & 5.19 & 5.79 & 6.46 & 7.19 & 7.99 & 8.86 & 9.82 & 10.91 & 11.98 & \\
\hline & $\%$ & 60.3 & 62.1 & 64.1 & 65.7 & 67.4 & 69.0 & 70.5 & 71.9 & 73.7 & \\
\hline & $\mathrm{T}$ & 172.9 & 166.4 & 160.4 & 155.6 & 151.2 & 147.5 & 144.3 & 141.3 & 139.1 & \\
\hline \multirow{7}{*}{$\begin{array}{c}90 \\
(217)\end{array}$} & C & 14105 & 16006 & 18126 & 20456 & 22992 & 25753 & 28834 & 31931 & & \\
\hline & $P$ & 2278 & 2313 & 2353 & 2383 & 2409 & 2429 & 2444 & 2457 & & \\
\hline & A & 11.25 & 11.39 & 11.55 & 11.67 & 11.77 & 11.86 & 11.90 & 11.96 & & \\
\hline & $M$ & 282.6 & 315.9 & 352.7 & 392.5 & 435.1 & 480.9 & 531.6 & 582.6 & & \\
\hline & $\mathrm{E}$ & 6.19 & 6.92 & 7.70 & 8.58 & 9.54 & 10.60 & 11.80 & 13.00 & & \\
\hline & $\%$ & 61.0 & 62.8 & 64.4 & 65.9 & 67.3 & 68.5 & 69.7 & 71.1 & & \\
\hline & $\mathrm{T}$ & 158.8 & 152.8 & 147.6 & 142.9 & 139.1 & 135.8 & 132.7 & 130.3 & & \\
\hline \multirow{7}{*}{$\begin{array}{c}80 \\
(188)\end{array}$} & C & 15276 & 17293 & 19574 & 22058 & 24784 & 27655 & 30848 & & & \\
\hline & $P$ & 2085 & 2116 & 2143 & 2168 & 2183 & 2200 & 2202 & & & \\
\hline & $A$ & 10.51 & 10.63 & 10.72 & 10.82 & 10.89 & 10.94 & 10.95 & & & \\
\hline & $M$ & 284.7 & 317.8 & 354.9 & 394.8 & 438.0 & 483.4 & 532.0 & & & \\
\hline & $E$ & 7.33 & 8.17 & 9.13 & 10.17 & 11.35 & 12.57 & 14.01 & & & \\
\hline & $\%$ & 61.2 & 62.7 & 64.2 & 65.1 & 66.2 & 66.9 & 67.6 & & & \\
\hline & $\mathrm{T}$ & 145.7 & 140.3 & 135.4 & 131.4 & 127.6 & 125.2 & 121.8 & & & \\
\hline \multirow{7}{*}{$\begin{array}{c}70 \\
(162)\end{array}$} & C & 16428 & 18627 & 21026 & 23659 & & & & & & \\
\hline & $P$ & 1905 & 1933 & 1956 & 1956 & & & & & & \\
\hline & A & 9.80 & 9.92 & 9.99 & 10.00 & & & & & & \\
\hline & $M$ & 286.6 & 321.0 & 358.8 & 396.8 & & & & & & \\
\hline & $E$ & 8.62 & 9.64 & 10.75 & 12.10 & & & & & & \\
\hline & $\%$ & 60.6 & 61.7 & 62.8 & 63.1 & & & & & & \\
\hline & T & 133.4 & 128.4 & 123.9 & 120.2 & & & & & & \\
\hline
\end{tabular}

C: Capacity (Btu/hr), P: Power (Watts), A: Current (Amps) at 230V, M: Mass Flow (lbs/hr), E: EER (Btu/Watt-hr) $\%$ : Isentropic Efficiency (\%), T: Discharge Temperature $\left({ }^{\circ} \mathrm{F}\right)$ 
Table A3: R-404A at $40^{\circ} \mathrm{F}$ Superheat, $0^{\circ} \mathrm{F}$ Subcooling

Suction Dew Point Temp, ${ }^{\circ} \mathrm{F}$ (Suction Pressure, psia)

\begin{tabular}{|c|c|c|c|c|c|c|c|c|c|c|c|}
\hline & & $-10(38)$ & $-5(42)$ & $0(47)$ & $5(52)$ & $10(57)$ & $15(63)$ & $20(69)$ & $25(76)$ & $30(84)$ & 35 (91) \\
\hline \multirow{7}{*}{$\begin{array}{c}140 \\
(416)\end{array}$} & $C$ & & & & 15109 & 17059 & 19211 & 21562 & 24136 & 26923 & 29925 \\
\hline & $P$ & & & & 3812 & 3872 & 3931 & 3984 & 4044 & 4104 & 4172 \\
\hline & $A$ & & & & 17.54 & 17.82 & 18.07 & 18.31 & 18.57 & 18.83 & 19.12 \\
\hline & $M$ & & & & 354.5 & 392.8 & 435.9 & 481.2 & 529.6 & 581.5 & 635.6 \\
\hline & $E$ & & & & 3.96 & 4.41 & 4.89 & 5.41 & 5.97 & 6.56 & 7.17 \\
\hline & $\%$ & & & & 60.8 & 63.2 & 65.1 & 67.2 & 69.0 & 70.8 & 72.4 \\
\hline & $\mathrm{T}$ & & & & 232.6 & 226.5 & 220.2 & 215.1 & 210.9 & 207.3 & 204.7 \\
\hline \multirow{7}{*}{$\begin{array}{c}130 \\
(368)\end{array}$} & C & & 13061 & 14865 & 16828 & 18984 & 21319 & 23868 & 26654 & 29708 & 32993 \\
\hline & $P$ & & 3340 & 3384 & 3445 & 3498 & 3554 & 3608 & 3662 & 3716 & 3770 \\
\hline & $A$ & & 15.55 & 15.74 & 16.01 & 16.23 & 16.47 & 16.70 & 16.94 & 17.15 & 17.38 \\
\hline & $M$ & & 289.0 & 323.0 & 360.3 & 399.7 & 441.7 & 486.9 & 536.0 & 588.6 & 643.6 \\
\hline & $\mathrm{E}$ & & 3.91 & 4.39 & 4.88 & 5.43 & 6.00 & 6.61 & 7.28 & 8.00 & 8.75 \\
\hline & $\%$ & & 59.1 & 61.5 & 63.8 & 65.8 & 67.9 & 69.7 & 71.4 & 73.0 & 74.6 \\
\hline & $\mathrm{T}$ & & 230.8 & 223.0 & 215.4 & 209.7 & 204.6 & 200.4 & 196.8 & 193.7 & 191.4 \\
\hline \multirow{7}{*}{$\begin{array}{c}120 \\
(324)\end{array}$} & $\mathrm{C}$ & 12697 & 14449 & 16346 & 18480 & 20800 & 23335 & 26092 & 29079 & 32396 & 35932 \\
\hline & $P$ & 2960 & 3013 & 3070 & 3125 & 3177 & 3230 & 3277 & 3318 & 3356 & 3398 \\
\hline & $A$ & 13.98 & 14.20 & 14.44 & 14.67 & 14.89 & 15.11 & 15.30 & 15.48 & 15.63 & 15.82 \\
\hline & $M$ & 262.1 & 293.5 & 327.5 & 364.6 & 404.0 & 446.8 & 492.4 & 541.4 & 594.9 & 650.7 \\
\hline & $\mathrm{E}$ & 4.29 & 4.80 & 5.32 & 5.91 & 6.55 & 7.22 & 7.96 & 8.76 & 9.65 & 10.57 \\
\hline & $\%$ & 59.7 & 61.7 & 64.0 & 66.1 & 68.0 & 69.8 & 71.5 & 73.0 & 74.7 & 76.5 \\
\hline & $\mathrm{T}$ & 220.8 & 213.5 & 206.1 & 200.1 & 195.0 & 190.7 & 187.0 & 183.7 & 180.7 & 178.4 \\
\hline \multirow{7}{*}{$\begin{array}{c}110 \\
(285)\end{array}$} & C & 13890 & 15745 & 17826 & 20097 & 22555 & 25313 & 28246 & 31510 & 35066 & \\
\hline & $P$ & 2700 & 2748 & 2801 & 2847 & 2894 & 2935 & 2970 & 3003 & 3028 & \\
\hline & A & 12.92 & 13.12 & 13.34 & 13.53 & 13.72 & 13.89 & 14.04 & 14.17 & 14.27 & \\
\hline & $M$ & 265.7 & 296.8 & 331.4 & 368.0 & 407.4 & 451.0 & 496.8 & 546.9 & 600.6 & \\
\hline & $E$ & 5.15 & 5.73 & 6.36 & 7.06 & 7.79 & 8.62 & 9.51 & 10.49 & 11.58 & \\
\hline & $\%$ & 61.8 & 63.8 & 65.9 & 67.5 & 69.5 & 71.0 & 72.5 & 74.0 & 75.8 & \\
\hline & $\mathrm{T}$ & 204.2 & 197.5 & 191.3 & 186.3 & 181.7 & 177.7 & 174.3 & 171.2 & 168.4 & \\
\hline \multirow{7}{*}{$\begin{array}{c}100 \\
(249)\end{array}$} & C & 15048 & 17029 & 19253 & 21656 & 24274 & 27205 & 30377 & 33842 & 37598 & \\
\hline & $P$ & 2464 & 2510 & 2556 & 2598 & 2633 & 2665 & 2691 & 2710 & 2731 & \\
\hline & A & 11.98 & 12.17 & 12.35 & 12.51 & 12.67 & 12.79 & 12.90 & 12.97 & 13.06 & \\
\hline & $M$ & 268.7 & 299.9 & 334.5 & 371.4 & 410.7 & 454.4 & 501.1 & 551.5 & 605.4 & \\
\hline & $E$ & 6.11 & 6.78 & 7.53 & 8.34 & 9.22 & 10.21 & 11.29 & 12.49 & 13.77 & \\
\hline & $\%$ & 63.3 & 65.1 & 66.8 & 68.5 & 70.1 & 71.7 & 72.8 & 74.2 & 75.8 & \\
\hline & $\mathrm{T}$ & 189.2 & 183.2 & 177.8 & 173.2 & 169.1 & 165.3 & 162.2 & 159.4 & 156.9 & \\
\hline \multirow{7}{*}{$\begin{array}{c}90 \\
(217)\end{array}$} & C & 16142 & 18277 & 20599 & 23194 & 26016 & 29050 & 32442 & 36012 & & \\
\hline & $P$ & 2254 & 2292 & 2329 & 2363 & 2390 & 2413 & 2428 & 2445 & & \\
\hline & $A$ & 11.17 & 11.30 & 11.45 & 11.59 & 11.70 & 11.79 & 11.85 & 11.93 & & \\
\hline & $M$ & 270.6 & 302.2 & 336.5 & 374.0 & 414.4 & 457.1 & 504.2 & 553.3 & & \\
\hline & $\mathrm{E}$ & 7.16 & 7.97 & 8.85 & 9.82 & 10.88 & 12.04 & 13.36 & 14.73 & & \\
\hline & $\%$ & 64.1 & 65.7 & 67.4 & 69.2 & 70.1 & 71.3 & 72.5 & 73.8 & & \\
\hline & $\mathrm{T}$ & 175.5 & 170.1 & 165.0 & 160.3 & 156.9 & 153.7 & 150.9 & 148.4 & & \\
\hline \multirow{7}{*}{$\begin{array}{c}80 \\
(188)\end{array}$} & C & 17259 & 19497 & 21987 & 24677 & 27621 & 30979 & 34522 & 38180 & & \\
\hline & $P$ & 2060 & 2092 & 2122 & 2146 & 2157 & 2190 & 2186 & 2199 & & \\
\hline & $A$ & 10.41 & 10.54 & 10.66 & 10.75 & 10.79 & 10.91 & 10.88 & 10.93 & & \\
\hline & $M$ & 273.0 & 304.5 & 339.3 & 376.4 & 416.3 & 461.4 & 508.6 & 556.8 & & \\
\hline & $\mathrm{E}$ & 8.38 & 9.32 & 10.36 & 11.50 & 12.80 & 14.14 & 15.79 & 17.37 & & \\
\hline & $\%$ & 64.5 & 65.8 & 67.3 & 68.3 & 69.5 & 70.1 & 70.6 & 71.3 & & \\
\hline & $\mathrm{T}$ & 162.4 & 157.4 & 152.6 & 148.9 & 145.2 & 143.2 & 139.7 & 137.8 & & \\
\hline \multirow{7}{*}{$\begin{array}{c}70 \\
(162)\end{array}$} & C & 18367 & 20741 & 23266 & 26217 & & & & & & \\
\hline & $P$ & 1880 & 1903 & 1915 & 1943 & & & & & & \\
\hline & A & 9.71 & 9.81 & 9.85 & 9.95 & & & & & & \\
\hline & $M$ & 274.9 & 307.0 & 339.9 & 379.1 & & & & & & \\
\hline & $\mathrm{E}$ & 9.77 & 10.90 & 12.15 & 13.50 & & & & & & \\
\hline & $\%$ & 64.1 & 65.0 & 66.1 & 67.2 & & & & & & \\
\hline & $\mathrm{T}$ & 150.0 & 145.3 & 141.1 & 137.4 & & & & & & \\
\hline
\end{tabular}

C: Capacity (Btu/hr), P: Power (Watts), A: Current (Amps) at 230V, M: Mass Flow (lbs/hr), E: EER (Btu/Watt-hr) $\%$ : Isentropic Efficiency (\%), T: Discharge Temperature $\left({ }^{\circ} \mathrm{F}\right)$ 
Table A4: ARM-31a at $65^{\circ} \mathrm{F}$ Suction Temperature, $0^{\circ} \mathrm{F}$ Subcooling

Suction Dew Point Temp, ${ }^{\circ} \mathrm{F}$ (Suction Pressure, psia)

\begin{tabular}{|c|c|c|c|c|c|c|c|c|c|c|c|}
\hline & & $-10(28)$ & $-5(31)$ & $0(35)$ & 5 (39) & $10(43)$ & $15(48)$ & $20(53)$ & $25(59)$ & $30(65)$ & $35(72)$ \\
\hline \multirow{7}{*}{$\begin{array}{c}140 \\
(361)\end{array}$} & C & & & & & & 16877 & 18867 & 21058 & 23350 & 25802 \\
\hline & $P$ & & & & & & 3385 & 3440 & 3498 & 3553 & 3614 \\
\hline & A & & & & & & 15.72 & 15.96 & 16.21 & 16.45 & 16.72 \\
\hline & $M$ & & & & & & 270.4 & 303.7 & 340.9 & 380.2 & 423.5 \\
\hline & $E$ & & & & & & 4.99 & 5.49 & 6.02 & 6.57 & 7.14 \\
\hline & $\%$ & & & & & & 66.8 & 68.4 & 69.8 & 70.9 & 71.2 \\
\hline & $T$ & & & & & & 260.9 & 248.4 & 237.1 & 226.8 & 218.1 \\
\hline \multirow{7}{*}{$\begin{array}{c}130 \\
(317)\end{array}$} & C & & & & 14513 & 16350 & 18325 & 20455 & 22804 & 25304 & 27929 \\
\hline & $P$ & & & & 2956 & 3004 & 3060 & 3111 & 3166 & 3214 & 3263 \\
\hline & A & & & & 13.93 & 14.14 & 14.37 & 14.59 & 14.82 & 15.02 & 15.24 \\
\hline & $M$ & & & & 215.4 & 243.5 & 274.1 & 307.4 & 344.5 & 384.4 & 427.3 \\
\hline & $E$ & & & & 4.91 & 5.44 & 5.99 & 6.57 & 7.20 & 7.87 & 8.56 \\
\hline & $\%$ & & & & 66.5 & 68.2 & 69.5 & 70.8 & 71.8 & 72.8 & 72.8 \\
\hline & $\mathrm{T}$ & & & & 267.6 & 254.3 & 242.2 & 231.1 & 220.7 & 211.1 & 203.1 \\
\hline \multirow{7}{*}{$\begin{array}{c}120 \\
(277)\end{array}$} & C & & & 13790 & 15582 & 17480 & 19591 & 21891 & 24379 & 27086 & 29892 \\
\hline & $P$ & & & 2625 & 2674 & 2723 & 2777 & 2822 & 2866 & 2906 & 2942 \\
\hline & A & & & 12.59 & 12.79 & 12.98 & 13.21 & 13.40 & 13.58 & 13.75 & 13.91 \\
\hline & $M$ & & & 191.9 & 217.6 & 245.0 & 275.7 & 309.4 & 346.1 & 386.6 & 429.5 \\
\hline & $E$ & & & 5.25 & 5.83 & 6.42 & 7.06 & 7.76 & 8.51 & 9.32 & 10.16 \\
\hline & $\%$ & & & 67.7 & 69.2 & 70.4 & 71.4 & 72.4 & 73.2 & 73.8 & 73.3 \\
\hline & $\mathrm{T}$ & & & 261.4 & 248.4 & 236.7 & 225.8 & 215.3 & 205.6 & 196.5 & 189.2 \\
\hline \multirow{7}{*}{$\begin{array}{c}110 \\
(241)\end{array}$} & C & 11538 & 13151 & 14876 & 16731 & 18798 & 21011 & 23462 & 26120 & 29027 & 32113 \\
\hline & $P$ & 2299 & 2339 & 2386 & 2428 & 2478 & 2519 & 2557 & 2591 & 2622 & 2647 \\
\hline & A & 11.30 & 11.45 & 11.65 & 11.81 & 12.01 & 12.18 & 12.33 & 12.47 & 12.60 & 12.70 \\
\hline & $M$ & 151.2 & 172.7 & 196.0 & 221.0 & 249.2 & 279.6 & 313.4 & 350.6 & 391.6 & 435.9 \\
\hline & $\mathrm{E}$ & 5.02 & 5.62 & 6.23 & 6.89 & 7.59 & 8.34 & 9.17 & 10.08 & 11.07 & 12.13 \\
\hline & $\%$ & 66.5 & 68.7 & 69.8 & 70.9 & 71.8 & 72.5 & 73.4 & 73.8 & 74.1 & 73.7 \\
\hline & $\mathrm{T}$ & 271.5 & 255.8 & 243.3 & 231.6 & 220.7 & 210.5 & 200.6 & 191.5 & 182.8 & 175.1 \\
\hline \multirow{7}{*}{$\begin{array}{c}100 \\
(209)\end{array}$} & C & 12353 & 14009 & 15816 & 17798 & 19954 & 22331 & 24940 & 27749 & 30722 & \\
\hline & P & 2088 & 2129 & 2169 & 2211 & 2250 & 2282 & 2314 & 2338 & 2360 & \\
\hline & A & 10.49 & 10.65 & 10.80 & 10.96 & 11.13 & 11.25 & 11.38 & 11.47 & 11.56 & \\
\hline & $M$ & 153.9 & 174.9 & 198.0 & 223.5 & 251.4 & 282.3 & 316.6 & 353.7 & 393.8 & \\
\hline & $E$ & 5.91 & 6.58 & 7.29 & 8.05 & 8.87 & 9.78 & 10.78 & 11.87 & 13.02 & \\
\hline & $\%$ & 68.7 & 70.3 & 71.2 & 72.0 & 72.6 & 73.1 & 73.5 & 73.4 & 72.9 & \\
\hline & $\mathrm{T}$ & 252.2 & 238.7 & 227.0 & 216.1 & 205.8 & 196.1 & 186.8 & 178.2 & 170.6 & \\
\hline \multirow{7}{*}{$\begin{array}{c}90 \\
(180)\end{array}$} & C & 13131 & 14833 & 16739 & 18824 & 21100 & 23601 & 26323 & 29216 & & \\
\hline & $\mathrm{P}$ & 1904 & 1937 & 1973 & 2008 & 2038 & 2064 & 2083 & 2104 & & \\
\hline & A & 9.79 & 9.91 & 10.04 & 10.18 & 10.31 & 10.41 & 10.49 & 10.57 & & \\
\hline & $M$ & 156.1 & 176.7 & 199.9 & 225.5 & 253.5 & 284.5 & 318.5 & 355.2 & & \\
\hline & $\mathrm{E}$ & 6.90 & 7.66 & 8.48 & 9.37 & 10.35 & 11.44 & 12.63 & 13.89 & & \\
\hline & $\%$ & 70.8 & 71.2 & 71.9 & 72.3 & 72.5 & 72.7 & 72.7 & 71.8 & & \\
\hline & $\mathrm{T}$ & 233.7 & 222.8 & 211.7 & 201.5 & 191.9 & 182.6 & 173.8 & 166.2 & & \\
\hline \multirow{7}{*}{$\begin{array}{c}80 \\
(155)\end{array}$} & C & 13800 & 15585 & 17582 & 19744 & 22151 & 24749 & 27488 & & & \\
\hline & $P$ & 1737 & 1768 & 1795 & 1818 & 1841 & 1858 & 1870 & & & \\
\hline & $A$ & 9.15 & 9.29 & 9.38 & 9.46 & 9.55 & 9.63 & 9.66 & & & \\
\hline & $M$ & 157.1 & 177.8 & 201.0 & 226.4 & 254.7 & 285.4 & 318.3 & & & \\
\hline & $\mathrm{E}$ & 7.95 & 8.82 & 9.79 & 10.86 & 12.03 & 13.32 & 14.70 & & & \\
\hline & $\%$ & 71.1 & 71.2 & 71.4 & 71.5 & 71.3 & 70.7 & 70.3 & & & \\
\hline & $\mathrm{T}$ & 218.6 & 208.3 & 198.0 & 188.3 & 179.1 & 170.4 & 162.2 & & & \\
\hline \multirow{7}{*}{$\begin{array}{c}70 \\
(132)\end{array}$} & C & 14448 & 16314 & 18389 & 20635 & & & & & & \\
\hline & $P$ & 1582 & 1606 & 1628 & 1643 & & & & & & \\
\hline & $A$ & 8.62 & 8.70 & 8.78 & 8.83 & & & & & & \\
\hline & $M$ & 157.9 & 178.6 & 201.8 & 226.9 & & & & & & \\
\hline & $\mathrm{E}$ & 9.13 & 10.16 & 11.30 & 12.56 & & & & & & \\
\hline & $\%$ & 70.1 & 69.8 & 69.7 & 69.2 & & & & & & \\
\hline & $\mathrm{T}$ & 205.4 & 195.4 & 185.6 & 176.4 & & & & & & \\
\hline
\end{tabular}

C: Capacity (Btu/hr), P: Power (Watts), A: Current (Amps) at 230V, M: Mass Flow (lbs/hr), E: EER (Btu/Watt-hr)

$\%$ : Isentropic Efficiency (\%), T: Discharge Temperature $\left({ }^{\circ} \mathrm{F}\right)$ 
Table A5: ARM-31a at $20^{\circ} \mathrm{F}$ Superheat, $0^{\circ} \mathrm{F}$ Subcooling

Suction Dew Point Temp, ${ }^{\circ} \mathrm{F}$ (Suction Pressure, psia)

\begin{tabular}{|c|c|c|c|c|c|c|c|c|c|c|c|}
\hline & & $-10(28)$ & $-5(31)$ & $0(35)$ & 5 (39) & $10(43)$ & $15(48)$ & $20(53)$ & $25(59)$ & $30(65)$ & $35(72)$ \\
\hline \multirow{7}{*}{$\begin{array}{c}140 \\
(361)\end{array}$} & $C$ & & & & 12366 & 14119 & 15996 & 18097 & 20331 & 22762 & 25388 \\
\hline & $P$ & & & & 3276 & 3326 & 3380 & 3440 & 3498 & 3555 & 3615 \\
\hline & $A$ & & & & 15.28 & 15.49 & 15.72 & 15.98 & 16.23 & 16.48 & 16.73 \\
\hline & $M$ & & & & 227.4 & 256.2 & 286.4 & 320.0 & 354.9 & 392.6 & 433.0 \\
\hline & $E$ & & & & 3.77 & 4.24 & 4.73 & 5.26 & 5.81 & 6.40 & 7.02 \\
\hline & $\%$ & & & & 58.1 & 60.8 & 63.0 & 65.1 & 67.0 & 68.7 & 70.0 \\
\hline & $\mathrm{T}$ & & & & 255.5 & 244.0 & 234.8 & 226.7 & 219.8 & 213.8 & 209.2 \\
\hline \multirow{7}{*}{$\begin{array}{c}130 \\
(317)\end{array}$} & C & & 10353 & 11934 & 13657 & 15514 & 17514 & 19738 & 22124 & 24754 & 27555 \\
\hline & $P$ & & 2859 & 2906 & 2955 & 3011 & 3064 & 3118 & 3170 & 3218 & 3266 \\
\hline & A & & 13.55 & 13.75 & 13.95 & 14.18 & 14.40 & 14.63 & 14.85 & 15.05 & 15.25 \\
\hline & $M$ & & 180.7 & 205.6 & 232.3 & 260.6 & 290.6 & 323.7 & 358.6 & 396.7 & 437.0 \\
\hline & $E$ & & 3.62 & 4.11 & 4.62 & 5.15 & 5.72 & 6.33 & 6.98 & 7.69 & 8.44 \\
\hline & $\%$ & & 56.0 & 58.7 & 61.2 & 63.3 & 65.3 & 67.1 & 68.8 & 70.4 & 71.5 \\
\hline & $\mathrm{T}$ & & 258.5 & 245.0 & 233.8 & 224.5 & 216.7 & 209.6 & 203.6 & 198.2 & 194.1 \\
\hline \multirow{7}{*}{$\begin{array}{c}120 \\
(277)\end{array}$} & $\mathrm{C}$ & 9809 & 11313 & 12943 & 14753 & 16718 & 18849 & 21202 & 23774 & 26578 & 29608 \\
\hline & $P$ & 2544 & 2584 & 2635 & 2685 & 2735 & 2786 & 2830 & 2873 & 2911 & 2947 \\
\hline & A & 12.27 & 12.43 & 12.65 & 12.85 & 13.05 & 13.27 & 13.45 & 13.62 & 13.79 & 13.93 \\
\hline & $M$ & 161.4 & 183.8 & 207.8 & 234.1 & 262.2 & 292.2 & 325.1 & 360.5 & 398.8 & 440.0 \\
\hline & $E$ & 3.86 & 4.38 & 4.91 & 5.49 & 6.11 & 6.77 & 7.49 & 8.27 & 9.13 & 10.05 \\
\hline & $\%$ & 56.5 & 58.9 & 61.1 & 63.1 & 64.9 & 66.7 & 68.4 & 69.9 & 71.3 & 72.3 \\
\hline & $\mathrm{T}$ & 248.4 & 235.7 & 224.9 & 215.6 & 207.5 & 200.5 & 194.1 & 188.6 & 183.6 & 179.7 \\
\hline \multirow{7}{*}{$\begin{array}{c}110 \\
(241)\end{array}$} & C & 10753 & 12305 & 14055 & 15933 & 18039 & 20299 & 22803 & 25550 & 28503 & \\
\hline & $P$ & 2305 & 2350 & 2398 & 2448 & 2493 & 2531 & 2568 & 2601 & 2629 & \\
\hline & A & 11.33 & 11.53 & 11.71 & 11.92 & 12.10 & 12.25 & 12.40 & 12.53 & 12.65 & \\
\hline & $M$ & 165.8 & 187.5 & 211.8 & 237.4 & 265.9 & 296.0 & 329.0 & 365.0 & 403.3 & \\
\hline & $E$ & 4.67 & 5.24 & 5.86 & 6.51 & 7.23 & 8.02 & 8.88 & 9.82 & 10.84 & \\
\hline & $\%$ & 58.7 & 60.6 & 62.5 & 64.3 & 65.9 & 67.5 & 68.9 & 70.2 & 71.2 & \\
\hline & $\mathrm{T}$ & 227.2 & 216.8 & 207.3 & 199.1 & 191.8 & 185.3 & 179.5 & 174.4 & 170.1 & \\
\hline \multirow{7}{*}{$\begin{array}{c}100 \\
(209)\end{array}$} & C & 11571 & 13222 & 15041 & 17061 & 19235 & 21639 & 24289 & 27179 & 30280 & \\
\hline & $P$ & 2100 & 2146 & 2191 & 2231 & 2267 & 2295 & 2323 & 2346 & 2360 & \\
\hline & A & 10.54 & 10.72 & 10.90 & 11.05 & 11.20 & 11.31 & 11.43 & 11.51 & 11.58 & \\
\hline & $M$ & 168.2 & 190.1 & 213.9 & 240.1 & 267.9 & 298.3 & 331.6 & 367.5 & 405.6 & \\
\hline & $E$ & 5.51 & 6.16 & 6.86 & 7.65 & 8.48 & 9.43 & 10.45 & 11.58 & 12.83 & \\
\hline & $\%$ & 60.0 & 61.6 & 63.2 & 64.8 & 66.3 & 67.7 & 68.8 & 69.9 & 70.7 & \\
\hline & $\mathrm{T}$ & 208.9 & 199.9 & 191.5 & 183.8 & 177.0 & 170.9 & 165.6 & 160.8 & 156.8 & \\
\hline \multirow{7}{*}{$\begin{array}{c}90 \\
(180)\end{array}$} & C & 12371 & 14124 & 16034 & 18163 & 20480 & 23018 & 25784 & 28680 & & \\
\hline & $P$ & 1925 & 1965 & 2001 & 2032 & 2058 & 2082 & 2097 & 2114 & & \\
\hline & A & 9.88 & 10.03 & 10.17 & 10.28 & 10.38 & 10.49 & 10.54 & 10.61 & & \\
\hline & $M$ & 170.3 & 192.4 & 216.2 & 242.5 & 270.8 & 301.5 & 334.4 & 368.9 & & \\
\hline & $E$ & 6.43 & 7.19 & 8.01 & 8.94 & 9.95 & 11.06 & 12.30 & 13.57 & & \\
\hline & $\%$ & 60.5 & 61.9 & 63.2 & 64.8 & 66.1 & 67.1 & 67.9 & 66.7 & & \\
\hline & $T$ & 192.7 & 184.2 & 176.6 & 169.1 & 162.7 & 157.2 & 152.4 & 150.2 & & \\
\hline \multirow{7}{*}{$\begin{array}{c}80 \\
(155)\end{array}$} & C & 13144 & 14974 & 16991 & 19210 & 21641 & 24310 & 27225 & 30493 & & \\
\hline & $P$ & 1763 & 1795 & 1823 & 1843 & 1863 & 1874 & 1884 & 1893 & & \\
\hline & A & 9.28 & 9.39 & 9.48 & 9.56 & 9.64 & 9.67 & 9.73 & 9.75 & & \\
\hline & $M$ & 172.0 & 194.1 & 218.1 & 244.2 & 272.6 & 303.4 & 336.7 & 374.3 & & \\
\hline & $\mathrm{E}$ & 7.46 & 8.34 & 9.32 & 10.42 & 11.61 & 12.97 & 14.45 & 16.11 & & \\
\hline & $\%$ & 60.3 & 61.5 & 62.8 & 64.0 & 64.9 & 65.6 & 66.0 & 66.5 & & \\
\hline & $\mathrm{T}$ & 177.4 & 169.5 & 161.9 & 155.2 & 149.4 & 144.2 & 139.6 & 135.6 & & \\
\hline \multirow{7}{*}{$\begin{array}{c}70 \\
(132)\end{array}$} & C & 13868 & 15785 & 17896 & 20159 & & & & & & \\
\hline & $P$ & 1609 & 1635 & 1657 & 1669 & & & & & & \\
\hline & A & 8.70 & 8.79 & 8.88 & 8.92 & & & & & & \\
\hline & $M$ & 173.2 & 195.3 & 219.4 & 244.9 & & & & & & \\
\hline & $\mathrm{E}$ & 8.62 & 9.66 & 10.80 & 12.08 & & & & & & \\
\hline & $\%$ & 59.3 & 60.3 & 61.2 & 61.8 & & & & & & \\
\hline & $T$ & 163.5 & 155.9 & 148.9 & 143.0 & & & & & & \\
\hline
\end{tabular}

C: Capacity (Btu/hr), P: Power (Watts), A: Current (Amps) at 230V, M: Mass Flow (lbs/hr), E: EER (Btu/Watt-hr) $\%$ : Isentropic Efficiency (\%), T: Discharge Temperature $\left({ }^{\circ} \mathrm{F}\right)$ 
Table A6: ARM-31a at $40^{\circ} \mathrm{F}$ Superheat, $0^{\circ} \mathrm{F}$ Subcooling

Suction Dew Point Temp, ${ }^{\circ} \mathrm{F}$ (Suction Pressure, psia)

\begin{tabular}{|c|c|c|c|c|c|c|c|c|c|c|c|}
\hline & & $-10(28)$ & $-5(31)$ & $0(35)$ & 5 (39) & $10(43)$ & $15(48)$ & $20(53)$ & $25(59)$ & $30(65)$ & $35(72)$ \\
\hline \multirow{7}{*}{$\begin{array}{c}140 \\
(361)\end{array}$} & C & & & & 13775 & 15696 & 17750 & 20013 & 22519 & 25163 & 27997 \\
\hline & $P$ & & & & 3282 & 3329 & 3379 & 3440 & 3498 & 3556 & 3615 \\
\hline & $A$ & & & & 15.28 & 15.49 & 15.72 & 15.97 & 16.21 & 16.46 & 16.72 \\
\hline & $M$ & & & & 218.9 & 246.3 & 275.1 & 306.6 & 340.9 & 376.6 & 414.3 \\
\hline & $E$ & & & & 4.20 & 4.72 & 5.25 & 5.82 & 6.44 & 7.08 & 7.74 \\
\hline & $\%$ & & & & 60.5 & 63.1 & 65.5 & 67.6 & 69.8 & 71.4 & 72.8 \\
\hline & $\mathrm{T}$ & & & & 272.9 & 261.7 & 252.4 & 244.4 & 237.1 & 231.4 & 226.8 \\
\hline \multirow{7}{*}{$\begin{array}{c}130 \\
(317)\end{array}$} & C & & 11464 & 13160 & 15017 & 17029 & 19189 & 21602 & 24219 & 27029 & 30044 \\
\hline & $P$ & & 2858 & 2904 & 2949 & 3004 & 3060 & 3114 & 3166 & 3215 & 3263 \\
\hline & $A$ & & 13.53 & 13.72 & 13.92 & 14.15 & 14.38 & 14.60 & 14.82 & 15.03 & 15.23 \\
\hline & $M$ & & 174.7 & 198.3 & 223.4 & 250.4 & 279.2 & 310.7 & 344.5 & 380.5 & 418.4 \\
\hline & $\mathrm{E}$ & & 4.01 & 4.53 & 5.09 & 5.67 & 6.27 & 6.94 & 7.65 & 8.41 & 9.21 \\
\hline & $\%$ & & 58.7 & 61.4 & 63.9 & 66.0 & 68.0 & 69.9 & 71.8 & 73.3 & 74.5 \\
\hline & $T$ & & 274.5 & 261.8 & 250.8 & 241.8 & 234.0 & 227.1 & 220.7 & 215.7 & 211.6 \\
\hline \multirow{7}{*}{$\begin{array}{c}120 \\
(277)\end{array}$} & $\mathrm{C}$ & 10764 & 12379 & 14153 & 16109 & 18177 & 20473 & 22977 & 25742 & 28732 & 31979 \\
\hline & $P$ & 2535 & 2576 & 2628 & 2678 & 2726 & 2780 & 2822 & 2866 & 2902 & 2940 \\
\hline & $A$ & 12.23 & 12.41 & 12.61 & 12.81 & 13.02 & 13.23 & 13.41 & 13.58 & 13.74 & 13.89 \\
\hline & $M$ & 156.3 & 177.7 & 200.9 & 226.0 & 252.1 & 281.2 & 312.0 & 346.1 & 382.4 & 421.3 \\
\hline & $E$ & 4.25 & 4.81 & 5.39 & 6.02 & 6.67 & 7.37 & 8.14 & 8.98 & 9.90 & 10.88 \\
\hline & $\%$ & 59.5 & 61.8 & 64.0 & 66.1 & 68.0 & 69.6 & 71.5 & 73.2 & 74.4 & 75.5 \\
\hline & $\mathrm{T}$ & 263.6 & 251.6 & 241.2 & 232.2 & 224.4 & 217.6 & 211.4 & 205.6 & 201.1 & 197.3 \\
\hline \multirow{7}{*}{$\begin{array}{c}110 \\
(241)\end{array}$} & C & 11710 & 13365 & 15208 & 17224 & 19433 & 21850 & 24527 & 27452 & 30573 & 34000 \\
\hline & $P$ & 2297 & 2344 & 2390 & 2436 & 2480 & 2523 & 2559 & 2591 & 2620 & 2644 \\
\hline & A & 11.30 & 11.48 & 11.67 & 11.85 & 12.02 & 12.20 & 12.35 & 12.47 & 12.59 & 12.70 \\
\hline & $M$ & 160.7 & 181.6 & 204.4 & 228.9 & 255.8 & 284.5 & 316.3 & 350.6 & 386.5 & 425.8 \\
\hline & $\mathrm{E}$ & 5.10 & 5.70 & 6.36 & 7.07 & 7.84 & 8.66 & 9.58 & 10.60 & 11.67 & 12.86 \\
\hline & $\%$ & 62.0 & 63.8 & 65.7 & 67.5 & 69.1 & 70.7 & 72.3 & 73.8 & 74.7 & 75.7 \\
\hline & $\mathrm{T}$ & 242.2 & 232.5 & 223.5 & 215.6 & 208.6 & 202.3 & 196.6 & 191.5 & 187.4 & 183.7 \\
\hline \multirow{7}{*}{$\begin{array}{c}100 \\
(209)\end{array}$} & C & 12500 & 14232 & 16171 & 18290 & 20582 & 23136 & 25934 & 29070 & 32250 & \\
\hline & $P$ & 2090 & 2136 & 2177 & 2216 & 2255 & 2284 & 2311 & 2338 & 2352 & \\
\hline & A & 10.50 & 10.68 & 10.84 & 10.98 & 11.15 & 11.26 & 11.37 & 11.47 & 11.54 & \\
\hline & $M$ & 163.0 & 183.8 & 206.5 & 231.3 & 257.8 & 286.9 & 318.5 & 353.7 & 388.9 & \\
\hline & $E$ & 5.98 & 6.66 & 7.43 & 8.25 & 9.13 & 10.13 & 11.22 & 12.43 & 13.71 & \\
\hline & $\%$ & 63.4 & 65.0 & 66.7 & 68.3 & 69.7 & 71.1 & 72.3 & 73.4 & 74.2 & \\
\hline & $\mathrm{T}$ & 224.1 & 215.4 & 207.3 & 200.1 & 193.8 & 187.9 & 182.8 & 178.2 & 174.5 & \\
\hline \multirow{7}{*}{$\begin{array}{c}90 \\
(180)\end{array}$} & C & 13290 & 15128 & 17151 & 19353 & 21807 & 24462 & 27392 & 30546 & & \\
\hline & $P$ & 1913 & 1949 & 1985 & 2015 & 2044 & 2065 & 2086 & 2104 & & \\
\hline & $A$ & 9.84 & 9.95 & 10.09 & 10.21 & 10.33 & 10.41 & 10.50 & 10.57 & & \\
\hline & $M$ & 165.3 & 186.0 & 209.0 & 233.7 & 260.8 & 289.8 & 321.6 & 355.2 & & \\
\hline & $\mathrm{E}$ & 6.95 & 7.76 & 8.64 & 9.60 & 10.67 & 11.84 & 13.13 & 14.52 & & \\
\hline & $\%$ & 64.1 & 65.6 & 67.1 & 68.4 & 69.7 & 70.7 & 71.5 & 71.8 & & \\
\hline & $\mathrm{T}$ & 207.7 & 199.6 & 192.1 & 185.6 & 179.6 & 174.4 & 169.7 & 166.2 & & \\
\hline \multirow{7}{*}{$\begin{array}{c}80 \\
(155)\end{array}$} & C & 14035 & 15959 & 18075 & 20400 & 22906 & 25715 & 28725 & & & \\
\hline & $P$ & 1745 & 1778 & 1807 & 1829 & 1847 & 1862 & 1873 & & & \\
\hline & A & 9.19 & 9.31 & 9.42 & 9.51 & 9.59 & 9.65 & 9.68 & & & \\
\hline & $M$ & 166.7 & 187.8 & 210.7 & 235.7 & 262.3 & 291.9 & 323.2 & & & \\
\hline & $\mathrm{E}$ & 8.04 & 8.98 & 10.01 & 11.16 & 12.40 & 13.81 & 15.34 & & & \\
\hline & $\%$ & 64.2 & 65.4 & 66.6 & 67.7 & 68.4 & 69.2 & 69.3 & & & \\
\hline & $\mathrm{T}$ & 192.3 & 184.9 & 178.0 & 171.8 & 166.6 & 161.5 & 157.9 & & & \\
\hline \multirow{7}{*}{$\begin{array}{c}70 \\
(132)\end{array}$} & C & 14671 & 16660 & 18847 & 21260 & & & & & & \\
\hline & $P$ & 1594 & 1619 & 1640 & 1653 & & & & & & \\
\hline & $A$ & 8.65 & 8.75 & 8.82 & 8.86 & & & & & & \\
\hline & $M$ & 168.1 & 189.2 & 212.1 & 237.1 & & & & & & \\
\hline & $\mathrm{E}$ & 9.20 & 10.29 & 11.49 & 12.86 & & & & & & \\
\hline & $\%$ & 63.3 & 64.2 & 65.1 & 65.8 & & & & & & \\
\hline & $\mathrm{T}$ & 178.5 & 171.4 & 165.0 & 159.0 & & & & & & \\
\hline
\end{tabular}

C: Capacity (Btu/hr), P: Power (Watts), A: Current (Amps) at 230V, M: Mass Flow (lbs/hr), E: EER (Btu/Watt-hr) $\%$ : Isentropic Efficiency (\%), T: Discharge Temperature $\left({ }^{\circ} \mathrm{F}\right)$ 
Table A7: D2Y- 65 at $65^{\circ} \mathrm{F}$ Suction Temperature, $0^{\circ} \mathrm{F}$ Subcooling

Suction Dew Point Temp, ${ }^{\circ} \mathrm{F}$ (Suction Pressure, psia)

\begin{tabular}{|c|c|c|c|c|c|c|c|c|c|c|c|}
\hline & & $-10(32)$ & $-5(36)$ & $0(40)$ & $5(45)$ & $10(50)$ & $15(55)$ & $20(61)$ & $25(67)$ & $30(74)$ & $35(81)$ \\
\hline \multirow{7}{*}{$\begin{array}{c}140 \\
(394)\end{array}$} & C & & & & & & 17604 & 19677 & 21897 & 24195 & 26568 \\
\hline & P & & & & & & 3756 & 3803 & 3858 & 3917 & 3988 \\
\hline & A & & & & & & 17.31 & 17.53 & 17.76 & 18.02 & 18.33 \\
\hline & $M$ & & & & & & 292.5 & 328.9 & 368.3 & 409.8 & 454.7 \\
\hline & $\mathrm{E}$ & & & & & & 4.69 & 5.17 & 5.68 & 6.18 & 6.66 \\
\hline & $\%$ & & & & & & 66.0 & 67.8 & 69.3 & 70.4 & 70.2 \\
\hline & $\mathrm{T}$ & & & & & & 265.0 & 251.9 & 240.2 & 230.0 & 222.0 \\
\hline \multirow{7}{*}{$\begin{array}{c}130 \\
(346)\end{array}$} & $C$ & & & & 15295 & 17229 & 19292 & 21514 & 23860 & 26422 & 29107 \\
\hline & $P$ & & & & 3288 & 3330 & 3373 & 3427 & 3482 & 3533 & 3587 \\
\hline & A & & & & 15.34 & 15.52 & 15.71 & 15.94 & 16.17 & 16.40 & 16.63 \\
\hline & $M$ & & & & 233.7 & 264.4 & 297.5 & 333.6 & 372.0 & 414.8 & 461.1 \\
\hline & $\mathrm{E}$ & & & & 4.65 & 5.17 & 5.72 & 6.28 & 6.85 & 7.48 & 8.11 \\
\hline & $\%$ & & & & 65.3 & 67.3 & 69.1 & 70.5 & 71.6 & 72.5 & 73.0 \\
\hline & $\mathrm{T}$ & & & & 272.7 & 258.1 & 245.2 & 233.7 & 223.3 & 213.6 & 204.9 \\
\hline \multirow{7}{*}{$\begin{array}{c}120 \\
(304)\end{array}$} & C & & & 14909 & 16765 & 18776 & 20943 & 23337 & 25907 & 28700 & 31662 \\
\hline & P & & & 2898 & 2942 & 2995 & 3052 & 3097 & 3143 & 3180 & 3218 \\
\hline & A & & & 13.73 & 13.91 & 14.13 & 14.37 & 14.56 & 14.76 & 14.92 & 15.08 \\
\hline & $M$ & & & 212.7 & 240.0 & 270.0 & 302.5 & 338.7 & 378.0 & 421.3 & 468.1 \\
\hline & $E$ & & & 5.15 & 5.70 & 6.27 & 6.86 & 7.54 & 8.24 & 9.03 & 9.84 \\
\hline & $\%$ & & & 67.5 & 69.1 & 70.3 & 71.1 & 72.4 & 73.2 & 74.0 & 74.3 \\
\hline & $\mathrm{T}$ & & & 264.0 & 250.7 & 238.8 & 228.2 & 217.2 & 207.5 & 198.1 & 189.7 \\
\hline \multirow{7}{*}{$\begin{array}{c}110 \\
(265)\end{array}$} & C & & 14044 & 15893 & 17888 & 20020 & 22363 & 24882 & 27630 & 30567 & \\
\hline & $P$ & & 2581 & 2622 & 2670 & 2716 & 2759 & 2798 & 2830 & 2860 & \\
\hline & A & & 12.45 & 12.61 & 12.81 & 13.00 & 13.17 & 13.34 & 13.47 & 13.60 & \\
\hline & $M$ & & 188.3 & 213.6 & 241.3 & 271.1 & 304.1 & 339.9 & 379.5 & 422.3 & \\
\hline & $E$ & & 5.44 & 6.06 & 6.70 & 7.37 & 8.11 & 8.89 & 9.76 & 10.69 & \\
\hline & $\%$ & & 67.5 & 69.3 & 70.7 & 71.7 & 72.6 & 73.3 & 73.8 & 74.0 & \\
\hline & $\mathrm{T}$ & & 260.3 & 246.2 & 233.7 & 222.6 & 212.1 & 202.3 & 193.0 & 184.4 & \\
\hline \multirow{7}{*}{$\begin{array}{c}100 \\
(230)\end{array}$} & C & 13278 & 15055 & 16998 & 19092 & 21348 & 23813 & 26514 & 29409 & 32459 & \\
\hline & $P$ & 2290 & 2335 & 2384 & 2423 & 2461 & 2492 & 2522 & 2548 & 2569 & \\
\hline & A & 11.30 & 11.48 & 11.68 & 11.83 & 11.99 & 12.11 & 12.22 & 12.34 & 12.41 & \\
\hline & $M$ & 168.2 & 191.2 & 216.6 & 244.1 & 273.9 & 306.7 & 343.1 & 382.4 & 424.7 & \\
\hline & $\mathrm{E}$ & 5.80 & 6.45 & 7.13 & 7.88 & 8.67 & 9.55 & 10.51 & 11.54 & 12.64 & \\
\hline & $\%$ & 68.4 & 69.9 & 71.0 & 72.1 & 72.6 & 73.2 & 73.4 & 73.6 & 73.2 & \\
\hline & $\mathrm{T}$ & 254.6 & 241.2 & 228.9 & 217.5 & 207.3 & 197.5 & 188.2 & 179.4 & 171.7 & \\
\hline \multirow{7}{*}{$\begin{array}{c}90 \\
(199)\end{array}$} & $C$ & 14163 & 16028 & 18049 & 20236 & 22633 & 25249 & 28073 & 31061 & & \\
\hline & $P$ & 2083 & 2126 & 2163 & 2192 & 2225 & 2248 & 2267 & 2288 & & \\
\hline & A & 10.52 & 10.67 & 10.83 & 10.92 & 11.07 & 11.15 & 11.23 & 11.31 & & \\
\hline & $M$ & 170.8 & 193.8 & 218.9 & 246.1 & 276.3 & 309.4 & 345.4 & 384.5 & & \\
\hline & $\mathrm{E}$ & 6.80 & 7.54 & 8.35 & 9.23 & 10.17 & 11.23 & 12.39 & 13.57 & & \\
\hline & $\%$ & 70.2 & 71.1 & 72.0 & 72.4 & 72.6 & 72.8 & 72.7 & 72.0 & & \\
\hline & $\mathrm{T}$ & 236.4 & 224.4 & 213.0 & 202.8 & 193.0 & 183.6 & 174.9 & 167.3 & & \\
\hline \multirow{7}{*}{$\begin{array}{c}80 \\
(172)\end{array}$} & C & 15006 & 16948 & 19067 & 21366 & 23876 & 26591 & 29567 & & & \\
\hline & $P$ & 1897 & 1930 & 1957 & 1981 & 1998 & 2022 & 2039 & & & \\
\hline & A & 9.80 & 9.92 & 10.02 & 10.11 & 10.18 & 10.28 & 10.34 & & & \\
\hline & $M$ & 172.9 & 195.8 & 220.8 & 248.2 & 278.1 & 311.4 & 348.2 & & & \\
\hline & $\mathrm{E}$ & 7.91 & 8.78 & 9.74 & 10.78 & 11.95 & 13.15 & 14.50 & & & \\
\hline & $\%$ & 71.0 & 71.7 & 72.0 & 72.0 & 71.5 & 71.3 & 70.7 & & & \\
\hline & $\mathrm{T}$ & 220.2 & 208.9 & 198.4 & 188.8 & 179.6 & 171.0 & 163.1 & & & \\
\hline \multirow{7}{*}{$\begin{array}{c}70 \\
(147)\end{array}$} & C & 15773 & 17784 & 19988 & 22490 & & & & & & \\
\hline & $P$ & 1724 & 1748 & 1769 & 1782 & & & & & & \\
\hline & A & 9.16 & 9.25 & 9.33 & 9.37 & & & & & & \\
\hline & M & 174.2 & 196.9 & 221.8 & 250.4 & & & & & & \\
\hline & E & 9.15 & 10.17 & 11.30 & 12.62 & & & & & & \\
\hline & $\%$ & 70.1 & 70.2 & 70.1 & 70.1 & & & & & & \\
\hline & $\mathrm{T}$ & 206.5 & 196.0 & 186.0 & 176.1 & & & & & & \\
\hline
\end{tabular}

C: Capacity (Btu/hr), P: Power (Watts), A: Current (Amps) at 230V, M: Mass Flow (lbs/hr), E: EER (Btu/Watt-hr) $\%$ : Isentropic Efficiency (\%), T: Discharge Temperature $\left({ }^{\circ} \mathrm{F}\right)$ 
Table A8: D2Y-65 at $20^{\circ} \mathrm{F}$ Superheat, $0^{\circ} \mathrm{F}$ Subcooling

Suction Dew Point Temp, ${ }^{\circ} \mathrm{F}$ (Suction Pressure, psia)

\begin{tabular}{|c|c|c|c|c|c|c|c|c|c|c|c|}
\hline & & $-10(32)$ & $-5(36)$ & $0(40)$ & $5(45)$ & $10(50)$ & $15(55)$ & $20(61)$ & $25(67)$ & $30(74)$ & $35(81)$ \\
\hline \multirow{7}{*}{$\begin{array}{c}140 \\
(394)\end{array}$} & $C$ & & & & 12936 & 14725 & 16688 & 18780 & 21075 & 23533 & 26153 \\
\hline & $P$ & & & & 3638 & 3680 & 3733 & 3790 & 3850 & 3910 & 3978 \\
\hline & $A$ & & & & 16.82 & 16.99 & 17.20 & 17.47 & 17.73 & 17.99 & 18.27 \\
\hline & $M$ & & & & 248.8 & 279.4 & 312.4 & 346.9 & 384.3 & 423.9 & 466.2 \\
\hline & $E$ & & & & 3.56 & 4.00 & 4.47 & 4.96 & 5.47 & 6.02 & 6.57 \\
\hline & $\%$ & & & & 57.3 & 60.3 & 62.7 & 64.7 & 66.6 & 68.2 & 69.5 \\
\hline & $\mathrm{T}$ & & & & 260.0 & 247.0 & 237.3 & 229.4 & 222.5 & 216.6 & 212.2 \\
\hline \multirow{7}{*}{$\begin{array}{c}130 \\
(346)\end{array}$} & C & & 10918 & 12626 & 14414 & 16334 & 18412 & 20690 & 23156 & 25833 & 28667 \\
\hline & $P$ & & 3177 & 3217 & 3259 & 3312 & 3367 & 3426 & 3479 & 3529 & 3579 \\
\hline & $A$ & & 14.86 & 15.03 & 15.21 & 15.44 & 15.67 & 15.92 & 16.15 & 16.36 & 16.58 \\
\hline & $M$ & & 197.8 & 225.7 & 254.5 & 284.7 & 316.9 & 351.9 & 389.3 & 429.3 & 471.7 \\
\hline & $\mathrm{E}$ & & 3.44 & 3.93 & 4.42 & 4.93 & 5.47 & 6.04 & 6.66 & 7.32 & 8.01 \\
\hline & $\%$ & & 55.3 & 58.4 & 61.1 & 63.3 & 65.2 & 67.0 & 68.7 & 70.1 & 71.3 \\
\hline & $\mathrm{T}$ & & 262.4 & 247.6 & 235.7 & 226.3 & 218.5 & 211.7 & 205.6 & 200.3 & 196.3 \\
\hline \multirow{7}{*}{$\begin{array}{c}120 \\
(304)\end{array}$} & $\mathrm{C}$ & 10503 & 12131 & 13896 & 15755 & 17794 & 20015 & 22451 & 24764 & 27805 & 30809 \\
\hline & $P$ & 2813 & 2859 & 2899 & 2949 & 3000 & 3054 & 3099 & 3142 & 3181 & 3219 \\
\hline & $A$ & 13.37 & 13.56 & 13.73 & 13.94 & 14.15 & 14.37 & 14.56 & 14.74 & 14.90 & 15.06 \\
\hline & $M$ & 178.4 & 203.5 & 230.1 & 257.9 & 287.8 & 320.1 & 355.0 & 387.3 & 430.3 & 472.4 \\
\hline & $\mathrm{E}$ & 3.73 & 4.24 & 4.79 & 5.34 & 5.93 & 6.55 & 7.24 & 7.88 & 8.74 & 9.57 \\
\hline & $\%$ & 55.9 & 58.6 & 61.2 & 63.3 & 65.1 & 66.8 & 68.4 & 69.9 & 71.2 & 72.6 \\
\hline & $\mathrm{T}$ & 251.6 & 237.9 & 226.1 & 216.6 & 208.7 & 201.8 & 195.6 & 190.2 & 185.3 & 181.0 \\
\hline \multirow{7}{*}{$\begin{array}{c}110 \\
(265)\end{array}$} & C & 11606 & 13275 & 15089 & 17058 & 19238 & 21610 & 24176 & 26990 & 30022 & \\
\hline & $P$ & 2538 & 2583 & 2634 & 2680 & 2726 & 2769 & 2804 & 2835 & 2865 & \\
\hline & $A$ & 12.26 & 12.44 & 12.65 & 12.84 & 13.02 & 13.20 & 13.34 & 13.47 & 13.60 & \\
\hline & $M$ & 183.8 & 207.8 & 233.5 & 261.0 & 291.2 & 323.6 & 358.1 & 395.8 & 435.9 & \\
\hline & $E$ & 4.57 & 5.14 & 5.73 & 6.36 & 7.06 & 7.80 & 8.62 & 9.52 & 10.48 & \\
\hline & $\%$ & 58.8 & 60.9 & 62.8 & 64.6 & 66.3 & 67.8 & 69.2 & 70.4 & 71.4 & \\
\hline & $\mathrm{T}$ & 228.3 & 217.3 & 208.0 & 199.8 & 192.5 & 186.2 & 180.6 & 175.6 & 171.5 & \\
\hline \multirow{7}{*}{$\begin{array}{c}100 \\
(230)\end{array}$} & C & 12528 & 14307 & 16209 & 18302 & 20593 & 23105 & 25831 & 28843 & 32112 & \\
\hline & $P$ & 2307 & 2358 & 2401 & 2442 & 2475 & 2506 & 2531 & 2553 & 2567 & \\
\hline & A & 11.35 & 11.55 & 11.72 & 11.89 & 12.02 & 12.15 & 12.25 & 12.33 & 12.39 & \\
\hline & $M$ & 186.3 & 210.5 & 235.9 & 263.6 & 293.5 & 325.9 & 360.8 & 399.0 & 440.3 & \\
\hline & $E$ & 5.43 & 6.07 & 6.75 & 7.50 & 8.32 & 9.22 & 10.20 & 11.30 & 12.51 & \\
\hline & $\%$ & 60.3 & 62.0 & 63.7 & 65.3 & 66.7 & 68.0 & 69.1 & 70.1 & 70.7 & \\
\hline & $\mathrm{T}$ & 209.5 & 199.9 & 191.7 & 184.0 & 177.5 & 171.6 & 166.5 & 161.7 & 157.8 & \\
\hline \multirow{7}{*}{$\begin{array}{c}90 \\
(199)\end{array}$} & C & 13361 & 15227 & 17269 & 19506 & 21921 & 24582 & 27462 & 30551 & & \\
\hline & $P$ & 2110 & 2153 & 2187 & 2220 & 2245 & 2266 & 2279 & 2291 & & \\
\hline & $A$ & 10.63 & 10.79 & 10.92 & 11.05 & 11.14 & 11.23 & 11.29 & 11.33 & & \\
\hline & $M$ & 187.6 & 211.6 & 237.5 & 265.6 & 295.5 & 328.3 & 363.3 & 400.6 & & \\
\hline & $\mathrm{E}$ & 6.33 & 7.07 & 7.90 & 8.79 & 9.77 & 10.85 & 12.05 & 13.34 & & \\
\hline & $\%$ & 60.5 & 62.2 & 63.8 & 65.2 & 66.3 & 67.3 & 67.9 & 68.3 & & \\
\hline & $\mathrm{T}$ & 193.6 & 184.6 & 176.5 & 169.4 & 163.3 & 157.8 & 153.2 & 149.3 & & \\
\hline \multirow{7}{*}{$\begin{array}{c}80 \\
(172)\end{array}$} & C & 14241 & 16198 & 18338 & 20694 & 23254 & 26011 & 29131 & 32207 & & \\
\hline & $P$ & 1929 & 1962 & 1990 & 2008 & 2023 & 2032 & 2043 & 2051 & & \\
\hline & $A$ & 9.95 & 10.05 & 10.18 & 10.23 & 10.29 & 10.32 & 10.37 & 10.40 & & \\
\hline & $M$ & 189.6 & 213.6 & 239.4 & 267.7 & 297.7 & 329.8 & 366.5 & 402.5 & & \\
\hline & $\mathrm{E}$ & 7.38 & 8.25 & 9.21 & 10.31 & 11.49 & 12.80 & 14.26 & 15.70 & & \\
\hline & $\%$ & 60.6 & 62.0 & 63.3 & 64.4 & 65.2 & 65.8 & 66.3 & 66.5 & & \\
\hline & $\mathrm{T}$ & 177.7 & 169.4 & 162.0 & 155.3 & 149.7 & 144.5 & 140.0 & 137.0 & & \\
\hline \multirow{7}{*}{$\begin{array}{c}70 \\
(147)\end{array}$} & C & 15105 & 17152 & 19381 & 21872 & & & & & & \\
\hline & $P$ & 1757 & 1781 & 1800 & 1810 & & & & & & \\
\hline & A & 9.29 & 9.38 & 9.44 & 9.47 & & & & & & \\
\hline & $M$ & 191.5 & 215.4 & 241.1 & 269.6 & & & & & & \\
\hline & $\mathrm{E}$ & 8.60 & 9.63 & 10.76 & 12.09 & & & & & & \\
\hline & $\%$ & 59.7 & 60.8 & 61.7 & 62.6 & & & & & & \\
\hline & $\mathrm{T}$ & 163.4 & 155.5 & 148.7 & 142.3 & & & & & & \\
\hline
\end{tabular}

C: Capacity (Btu/hr), P: Power (Watts), A: Current (Amps) at 230V, M: Mass Flow (lbs/hr), E: EER (Btu/Watt-hr) $\%$ : Isentropic Efficiency (\%), T: Discharge Temperature $\left({ }^{\circ} \mathrm{F}\right)$ 
Table A9: D2Y-65 at $40^{\circ} \mathrm{F}$ Superheat, $0^{\circ} \mathrm{F}$ Subcooling

Suction Dew Point Temp, ${ }^{\circ} \mathrm{F}$ (Suction Pressure, psia)

\begin{tabular}{|c|c|c|c|c|c|c|c|c|c|c|c|}
\hline & & $-10(32)$ & $-5(36)$ & $0(40)$ & $5(45)$ & $10(50)$ & $15(55)$ & $20(61)$ & $25(67)$ & $30(74)$ & $35(81)$ \\
\hline \multirow{7}{*}{$\begin{array}{c}140 \\
(394)\end{array}$} & $C$ & & & & & 17114 & 19346 & 21743 & 24366 & 27170 & 30321 \\
\hline & $P$ & & & & & 3693 & 3738 & 3790 & 3858 & 3911 & 3968 \\
\hline & $A$ & & & & & 17.02 & 17.22 & 17.44 & 17.76 & 17.98 & 18.22 \\
\hline & $M$ & & & & & 268.2 & 299.6 & 332.7 & 368.3 & 406.4 & 448.3 \\
\hline & $E$ & & & & & 4.63 & 5.18 & 5.74 & 6.32 & 6.95 & 7.64 \\
\hline & $\%$ & & & & & 62.3 & 65.0 & 67.1 & 69.3 & 71.0 & 73.7 \\
\hline & $\mathrm{T}$ & & & & & 265.8 & 255.5 & 247.2 & 240.2 & 234.2 & 227.7 \\
\hline \multirow{7}{*}{$\begin{array}{c}130 \\
(346)\end{array}$} & C & & & 14483 & 16472 & 18615 & 20961 & 23488 & 26219 & 29213 & 32420 \\
\hline & $P$ & & & 3216 & 3257 & 3307 & 3365 & 3419 & 3482 & 3524 & 3577 \\
\hline & $A$ & & & 15.01 & 15.20 & 15.41 & 15.65 & 15.88 & 16.17 & 16.33 & 16.56 \\
\hline & $M$ & & & 217.5 & 244.5 & 273.1 & 304.0 & 337.0 & 372.0 & 410.4 & 450.7 \\
\hline & $E$ & & & 4.50 & 5.06 & 5.63 & 6.23 & 6.87 & 7.53 & 8.29 & 9.06 \\
\hline & $\%$ & & & 60.9 & 63.5 & 65.7 & 67.7 & 69.6 & 71.6 & 72.8 & 74.1 \\
\hline & $\mathrm{T}$ & & & 264.9 & 253.6 & 244.3 & 236.3 & 229.5 & 223.3 & 218.2 & 214.2 \\
\hline \multirow{7}{*}{$\begin{array}{c}120 \\
(304)\end{array}$} & $\mathrm{C}$ & 11971 & 13765 & 15698 & 17737 & 19987 & 22464 & 25135 & 28028 & 31233 & 34647 \\
\hline & $P$ & 2800 & 2848 & 2893 & 2942 & 2993 & 3047 & 3089 & 3142 & 3175 & 3213 \\
\hline & $A$ & 13.30 & 13.50 & 13.69 & 13.89 & 14.11 & 14.32 & 14.51 & 14.75 & 14.86 & 15.02 \\
\hline & M & 172.8 & 196.5 & 221.7 & 247.9 & 276.2 & 307.3 & 340.2 & 375.5 & 414.5 & 455.1 \\
\hline & $\mathrm{E}$ & 4.28 & 4.83 & 5.43 & 6.03 & 6.68 & 7.37 & 8.14 & 8.92 & 9.84 & 10.78 \\
\hline & $\%$ & 58.9 & 61.5 & 64.0 & 66.0 & 67.8 & 69.6 & 71.3 & 73.0 & 74.2 & 75.6 \\
\hline & $\mathrm{T}$ & 267.2 & 254.2 & 243.1 & 234.0 & 226.3 & 219.3 & 213.0 & 207.7 & 203.0 & 199.0 \\
\hline \multirow{7}{*}{$\begin{array}{c}110 \\
(265)\end{array}$} & C & 13040 & 14869 & 16861 & 18988 & 21382 & 23978 & 26784 & 29874 & 33222 & 36850 \\
\hline & $P$ & 2530 & 2577 & 2624 & 2671 & 2718 & 2760 & 2793 & 2830 & 2854 & 2874 \\
\hline & $A$ & 12.22 & 12.42 & 12.59 & 12.80 & 12.98 & 13.15 & 13.30 & 13.47 & 13.54 & 13.62 \\
\hline & $M$ & 177.9 & 200.8 & 225.3 & 251.1 & 279.8 & 310.8 & 343.7 & 379.5 & 418.2 & 459.6 \\
\hline & $E$ & 5.15 & 5.77 & 6.43 & 7.11 & 7.87 & 8.69 & 9.59 & 10.55 & 11.64 & 12.82 \\
\hline & $\%$ & 61.8 & 64.0 & 65.9 & 67.5 & 69.3 & 70.8 & 72.3 & 73.8 & 74.6 & 76.1 \\
\hline & $\mathrm{T}$ & 244.3 & 233.6 & 224.5 & 217.0 & 209.9 & 203.6 & 198.1 & 193.0 & 188.9 & 184.7 \\
\hline \multirow{7}{*}{$\begin{array}{c}100 \\
(230)\end{array}$} & C & 13944 & 15843 & 17917 & 20174 & 22674 & 25407 & 28357 & 31619 & 35025 & \\
\hline & $P$ & 2296 & 2342 & 2388 & 2426 & 2462 & 2495 & 2517 & 2548 & 2556 & \\
\hline & A & 11.30 & 11.48 & 11.67 & 11.82 & 11.96 & 12.09 & 12.18 & 12.34 & 12.34 & \\
\hline & $M$ & 180.6 & 203.0 & 227.4 & 253.4 & 282.1 & 313.2 & 346.4 & 382.4 & 419.8 & \\
\hline & $E$ & 6.07 & 6.77 & 7.50 & 8.31 & 9.21 & 10.18 & 11.26 & 12.41 & 13.70 & \\
\hline & $\%$ & 63.6 & 65.4 & 66.9 & 68.3 & 69.9 & 71.2 & 72.3 & 73.6 & 74.2 & \\
\hline & $\mathrm{T}$ & 225.1 & 216.0 & 208.2 & 201.3 & 194.7 & 189.0 & 183.9 & 179.4 & 175.9 & \\
\hline \multirow{7}{*}{$\begin{array}{c}90 \\
(199)\end{array}$} & C & 14731 & 16733 & 18915 & 21317 & 23935 & 26795 & 29924 & 33439 & & \\
\hline & $P$ & 2094 & 2136 & 2171 & 2203 & 2228 & 2252 & 2268 & 2282 & & \\
\hline & $A$ & 10.55 & 10.72 & 10.86 & 10.98 & 11.08 & 11.17 & 11.23 & 11.28 & & \\
\hline & $M$ & 181.6 & 204.4 & 228.9 & 255.4 & 284.2 & 315.3 & 349.0 & 386.5 & & \\
\hline & $\mathrm{E}$ & 7.04 & 7.83 & 8.71 & 9.68 & 10.74 & 11.90 & 13.19 & 14.65 & & \\
\hline & $\%$ & 64.1 & 65.8 & 67.2 & 68.5 & 69.7 & 70.7 & 71.6 & 73.2 & & \\
\hline & $T$ & 209.0 & 200.4 & 193.0 & 186.5 & 180.5 & 175.4 & 170.6 & 165.8 & & \\
\hline \multirow{7}{*}{$\begin{array}{c}80 \\
(172)\end{array}$} & C & 15256 & 17300 & 19534 & 21986 & 24663 & 27537 & 30973 & 34191 & & \\
\hline & $P$ & 1914 & 1944 & 1972 & 1992 & 2005 & 2029 & 2040 & 2029 & & \\
\hline & $A$ & 9.89 & 9.98 & 10.11 & 10.18 & 10.21 & 10.32 & 10.34 & 10.30 & & \\
\hline & $M$ & 183.8 & 206.5 & 231.0 & 257.7 & 286.4 & 316.8 & 353.0 & 386.4 & & \\
\hline & $\mathrm{E}$ & 7.97 & 8.90 & 9.90 & 11.04 & 12.30 & 13.57 & 15.18 & 16.85 & & \\
\hline & $\%$ & 64.4 & 65.9 & 66.8 & 67.8 & 68.7 & 69.4 & 71.6 & 69.9 & & \\
\hline & $\mathrm{T}$ & 192.9 & 185.0 & 178.6 & 172.5 & 166.9 & 162.7 & 157.0 & 154.5 & & \\
\hline \multirow{7}{*}{$\begin{array}{c}70 \\
(147)\end{array}$} & C & 15971 & 18084 & 20392 & 23001 & & & & & & \\
\hline & $P$ & 1740 & 1763 & 1782 & 1795 & & & & & & \\
\hline & $A$ & 9.22 & 9.31 & 9.38 & 9.42 & & & & & & \\
\hline & $M$ & 185.5 & 208.1 & 232.6 & 260.1 & & & & & & \\
\hline & $\mathrm{E}$ & 9.18 & 10.26 & 11.44 & 12.82 & & & & & & \\
\hline & $\%$ & 63.6 & 64.7 & 65.4 & 66.5 & & & & & & \\
\hline & $\mathrm{T}$ & 178.8 & 171.6 & 165.3 & 159.1 & & & & & & \\
\hline
\end{tabular}

C: Capacity (Btu/hr), P: Power (Watts), A: Current (Amps) at 230V, M: Mass Flow (lbs/hr), E: EER (Btu/Watt-hr) $\%$ : Isentropic Efficiency (\%), T: Discharge Temperature $\left({ }^{\circ} \mathrm{F}\right)$ 
Table A10: L-40 at $65^{\circ} \mathrm{F}$ Suction Temperature, $0^{\circ} \mathrm{F}$ Subcooling

Suction Dew Point Temp, ${ }^{\circ} \mathrm{F}$ (Suction Pressure, psia)

\begin{tabular}{|c|c|c|c|c|c|c|c|c|c|c|c|}
\hline & & $-10(27)$ & $-5(30)$ & $0(34)$ & $5(38)$ & $10(43)$ & $15(47)$ & $20(53)$ & $25(58)$ & $30(64)$ & $35(71)$ \\
\hline \multirow{7}{*}{$\begin{array}{c}140 \\
(362)\end{array}$} & C & & & & & & & 18978 & 21243 & 23662 & 26389 \\
\hline & $\mathrm{P}$ & & & & & & & 3499 & 3547 & 3596 & 3641 \\
\hline & A & & & & & & & 16.21 & 16.42 & 16.64 & 16.82 \\
\hline & $M$ & & & & & & & 262.4 & 295.2 & 330.8 & 371.4 \\
\hline & $\mathrm{E}$ & & & & & & & 5.42 & 5.99 & 6.58 & 7.25 \\
\hline & $\%$ & & & & & & & 70.5 & 72.6 & 74.1 & 76.2 \\
\hline & $T$ & & & & & & & 267.0 & 253.1 & 241.2 & 229.0 \\
\hline \multirow{7}{*}{$\begin{array}{c}130 \\
(317)\end{array}$} & $C$ & & & & & 16283 & 18336 & 20521 & 22914 & 25495 & 28246 \\
\hline & $P$ & & & & & 3057 & 3098 & 3144 & 3193 & 3239 & 3285 \\
\hline & A & & & & & 14.38 & 14.55 & 14.75 & 14.97 & 15.17 & 15.37 \\
\hline & $M$ & & & & & 209.3 & 236.7 & 266.2 & 298.8 & 334.3 & 372.7 \\
\hline & $\mathrm{E}$ & & & & & 5.33 & 5.92 & 6.53 & 7.18 & 7.87 & 8.60 \\
\hline & $\%$ & & & & & 70.2 & 71.8 & 73.4 & 74.7 & 75.9 & 76.7 \\
\hline & T & & & & & 274.1 & 260.3 & 247.3 & 235.4 & 224.3 & 214.3 \\
\hline \multirow{7}{*}{$\begin{array}{c}120 \\
(277)\end{array}$} & C & & & & 15600 & 17585 & 19728 & 22052 & 24585 & 27322 & 30298 \\
\hline & $P$ & & & & 2712 & 2749 & 2792 & 2838 & 2882 & 2921 & 2952 \\
\hline & A & & & & 12.98 & 13.13 & 13.32 & 13.50 & 13.69 & 13.85 & 13.98 \\
\hline & $M$ & & & & 188.7 & 213.4 & 240.5 & 270.0 & 302.4 & 337.8 & 376.8 \\
\hline & $\mathrm{E}$ & & & & 5.75 & 6.40 & 7.07 & 7.77 & 8.53 & 9.35 & 10.26 \\
\hline & $\%$ & & & & 71.9 & 73.5 & 74.8 & 75.7 & 76.7 & 77.5 & 78.2 \\
\hline & T & & & & 266.5 & 252.7 & 240.3 & 228.9 & 218.0 & 207.9 & 198.0 \\
\hline \multirow{7}{*}{$\begin{array}{c}110 \\
(241)\end{array}$} & C & & & 14682 & 16580 & 18649 & 20891 & 23348 & 26025 & 28968 & 31984 \\
\hline & $P$ & & & 2399 & 2440 & 2483 & 2524 & 2562 & 2595 & 2622 & 2649 \\
\hline & A & & & 11.73 & 11.90 & 12.07 & 12.24 & 12.39 & 12.53 & 12.64 & 12.75 \\
\hline & $M$ & & & 168.2 & 190.5 & 215.1 & 241.8 & 271.3 & 303.9 & 339.9 & 377.5 \\
\hline & $E$ & & & 6.12 & 6.79 & 7.51 & 8.28 & 9.11 & 10.03 & 11.05 & 12.08 \\
\hline & $\%$ & & & 72.0 & 73.4 & 74.5 & 75.4 & 76.2 & 76.8 & 77.3 & 76.2 \\
\hline & $T$ & & & 262.1 & 248.5 & 236.1 & 224.6 & 213.7 & 203.4 & 193.4 & 185.6 \\
\hline \multirow{7}{*}{$\begin{array}{c}100 \\
(209)\end{array}$} & $C$ & 12075 & 13849 & 15641 & 17629 & 19808 & 22173 & 24750 & 27582 & 30483 & \\
\hline & $P$ & 2103 & 2140 & 2172 & 2211 & 2247 & 2281 & 2309 & 2332 & 2356 & \\
\hline & A & 10.58 & 10.74 & 10.85 & 11.01 & 11.15 & 11.29 & 11.40 & 11.49 & 11.58 & \\
\hline & $M$ & 131.2 & 150.9 & 170.8 & 193.1 & 217.7 & 244.5 & 274.0 & 306.7 & 341.0 & \\
\hline & $E$ & 5.74 & 6.47 & 7.20 & 7.97 & 8.82 & 9.72 & 10.72 & 11.83 & 12.94 & \\
\hline & $\%$ & 71.2 & 74.0 & 74.8 & 75.7 & 76.3 & 76.7 & 76.9 & 76.8 & 74.8 & \\
\hline & $\mathrm{T}$ & 271.5 & 254.1 & 241.5 & 229.5 & 218.3 & 207.7 & 197.8 & 188.3 & 181.5 & \\
\hline \multirow{7}{*}{$\begin{array}{c}90 \\
(180)\end{array}$} & $C$ & 12833 & 14613 & 16517 & 18598 & 20877 & 23380 & 26096 & 28878 & & \\
\hline & $P$ & 1901 & 1935 & 1969 & 1999 & 2030 & 2053 & 2072 & 2094 & & \\
\hline & A & 9.83 & 9.95 & 10.07 & 10.18 & 10.33 & 10.40 & 10.48 & 10.56 & & \\
\hline & $M$ & 133.5 & 152.3 & 172.6 & 194.9 & 219.5 & 246.6 & 276.3 & 307.5 & & \\
\hline & $\mathrm{E}$ & 6.75 & 7.55 & 8.39 & 9.30 & 10.28 & 11.39 & 12.59 & 13.79 & & \\
\hline & $\%$ & 73.3 & 75.4 & 75.7 & 76.2 & 76.3 & 76.4 & 75.9 & 73.4 & & \\
\hline & T & 251.1 & 236.2 & 224.6 & 213.4 & 203.0 & 192.9 & 183.7 & 177.2 & & \\
\hline \multirow{7}{*}{$\begin{array}{c}80 \\
(154)\end{array}$} & C & 13590 & 15379 & 17351 & 19546 & 21932 & 24568 & 27407 & & & \\
\hline & $P$ & 1729 & 1755 & 1786 & 1806 & 1828 & 1842 & 1848 & & & \\
\hline & A & 9.18 & 9.27 & 9.41 & 9.48 & 9.58 & 9.62 & 9.63 & & & \\
\hline & $M$ & 135.7 & 153.9 & 174.0 & 196.6 & 221.2 & 248.6 & 278.2 & & & \\
\hline & $E$ & 7.86 & 8.76 & 9.72 & 10.82 & 12.00 & 13.34 & 14.83 & & & \\
\hline & $\%$ & 75.5 & 75.7 & 75.7 & 75.8 & 75.5 & 74.6 & 73.8 & & & \\
\hline & $\mathrm{T}$ & 231.1 & 219.8 & 208.9 & 198.2 & 188.4 & 179.0 & 169.9 & & & \\
\hline \multirow{7}{*}{$\begin{array}{c}70 \\
(131)\end{array}$} & C & 14185 & 16053 & 18122 & 20401 & & & & & & \\
\hline & $\mathrm{P}$ & 1570 & 1592 & 1612 & 1627 & & & & & & \\
\hline & A & 8.61 & 8.70 & 8.76 & 8.82 & & & & & & \\
\hline & $M$ & 136.3 & 154.6 & 174.9 & 197.4 & & & & & & \\
\hline & $E$ & 9.04 & 10.08 & 11.24 & 12.54 & & & & & & \\
\hline & $\%$ & 74.0 & 73.9 & 73.5 & 72.9 & & & & & & \\
\hline & T & 217.4 & 206.4 & 195.9 & 185.6 & & & & & & \\
\hline
\end{tabular}

C: Capacity (Btu/hr), P: Power (Watts), A: Current (Amps) at 230V, M: Mass Flow (lbs/hr), E: EER (Btu/Watt-hr) $\%$ : Isentropic Efficiency (\%), T: Discharge Temperature $\left({ }^{\circ} \mathrm{F}\right)$ 
Table A11: L-40 at $20^{\circ} \mathrm{F}$ Superheat, $0^{\circ} \mathrm{F}$ Subcooling

Suction Dew Point Temp, ${ }^{\circ} \mathrm{F}$ (Suction Pressure, psia)

\begin{tabular}{|c|c|c|c|c|c|c|c|c|c|c|c|}
\hline & & $-10(27)$ & $-5(30)$ & $0(34)$ & $5(38)$ & $10(43)$ & $15(47)$ & $20(53)$ & $25(58)$ & $30(64)$ & 35 (71) \\
\hline \multirow{7}{*}{$\begin{array}{c}140 \\
(362)\end{array}$} & C & & & & & 14282 & 16282 & 18440 & 20753 & 23255 & 25918 \\
\hline & $P$ & & & & & 3405 & 3447 & 3494 & 3541 & 3594 & 3655 \\
\hline & A & & & & & 15.84 & 16.03 & 16.22 & 16.43 & 16.66 & 16.92 \\
\hline & $M$ & & & & & 219.0 & 247.0 & 276.7 & 308.3 & 342.1 & 378.0 \\
\hline & $E$ & & & & & 4.19 & 4.72 & 5.28 & 5.86 & 6.47 & 7.09 \\
\hline & $\%$ & & & & & 62.4 & 65.2 & 67.9 & 70.0 & 72.1 & 73.8 \\
\hline & T & & & & & 266.2 & 253.9 & 243.3 & 235.0 & 227.6 & 221.7 \\
\hline \multirow{7}{*}{$\begin{array}{c}130 \\
(317)\end{array}$} & C & & & 11948 & 13766 & 15721 & 17796 & 20040 & 22543 & 25222 & 28052 \\
\hline & $P$ & & & 2952 & 3011 & 3053 & 3095 & 3138 & 3187 & 3238 & 3290 \\
\hline & A & & & 13.92 & 14.21 & 14.38 & 14.56 & 14.74 & 14.95 & 15.16 & 15.38 \\
\hline & M & & & 174.4 & 198.8 & 224.7 & 251.7 & 280.6 & 312.7 & 346.6 & 382.4 \\
\hline & $E$ & & & 4.05 & 4.57 & 5.15 & 5.75 & 6.39 & 7.07 & 7.79 & 8.53 \\
\hline & $\%$ & & & 60.0 & 63.2 & 65.9 & 68.3 & 70.5 & 72.3 & 74.2 & 75.6 \\
\hline & $\mathrm{T}$ & & & 270.1 & 254.8 & 242.7 & 232.7 & 224.2 & 216.7 & 210.0 & 205.1 \\
\hline \multirow{7}{*}{$\begin{array}{c}120 \\
(277)\end{array}$} & C & & 11437 & 13134 & 15030 & 17052 & 19239 & 21640 & 24265 & 27120 & 30132 \\
\hline & $P$ & & 2611 & 2652 & 2715 & 2751 & 2791 & 2837 & 2880 & 2918 & 2956 \\
\hline & $A$ & & 12.55 & 12.71 & 13.00 & 13.15 & 13.31 & 13.50 & 13.68 & 13.83 & 14.00 \\
\hline & $M$ & & 158.0 & 179.6 & 203.6 & 228.7 & 255.6 & 284.8 & 316.5 & 350.7 & 386.7 \\
\hline & $E$ & & 4.38 & 4.95 & 5.54 & 6.20 & 6.89 & 7.63 & 8.43 & 9.29 & 10.19 \\
\hline & $\%$ & & 61.1 & 63.3 & 66.0 & 68.3 & 70.4 & 72.0 & 73.9 & 75.4 & 76.9 \\
\hline & $\mathrm{T}$ & & 257.6 & 245.5 & 232.7 & 222.6 & 214.0 & 206.7 & 199.8 & 194.0 & 189.2 \\
\hline \multirow{7}{*}{$\begin{array}{c}110 \\
(241)\end{array}$} & $C$ & 10761 & 12429 & 14181 & 16153 & 18239 & 20578 & 23119 & 25910 & 28861 & \\
\hline & $P$ & 2315 & 2358 & 2395 & 2445 & 2486 & 2529 & 2563 & 2595 & 2626 & \\
\hline & $A$ & 11.38 & 11.56 & 11.70 & 11.93 & 12.09 & 12.26 & 12.40 & 12.53 & 12.65 & \\
\hline & M & 141.5 & 161.9 & 183.0 & 206.5 & 231.0 & 258.3 & 287.7 & 319.7 & 353.4 & \\
\hline & $E$ & 4.65 & 5.27 & 5.92 & 6.61 & 7.34 & 8.14 & 9.02 & 9.98 & 10.99 & \\
\hline & $\%$ & 61.0 & 63.8 & 65.6 & 68.0 & 69.7 & 71.4 & 73.1 & 74.4 & 75.5 & \\
\hline & $\mathrm{T}$ & 249.0 & 234.4 & 224.0 & 213.2 & 205.1 & 197.4 & 190.4 & 184.3 & 179.5 & \\
\hline \multirow{7}{*}{$\begin{array}{c}100 \\
(209)\end{array}$} & $C$ & 11643 & 13309 & 15139 & 17212 & 19412 & 21869 & 24528 & 27461 & 30611 & \\
\hline & $P$ & 2102 & 2139 & 2181 & 2222 & 2256 & 2286 & 2314 & 2337 & 2350 & \\
\hline & $A$ & 10.58 & 10.72 & 10.90 & 11.05 & 11.19 & 11.30 & 11.40 & 11.50 & 11.56 & \\
\hline & $M$ & 145.1 & 164.3 & 185.2 & 208.7 & 233.3 & 260.6 & 289.9 & 322.0 & 356.2 & \\
\hline & $E$ & 5.54 & 6.22 & 6.94 & 7.75 & 8.60 & 9.57 & 10.60 & 11.75 & 13.03 & \\
\hline & $\%$ & 63.3 & 65.2 & 66.9 & 68.5 & 70.1 & 71.5 & 72.8 & 73.8 & 74.3 & \\
\hline & $\mathrm{T}$ & 225.9 & 215.0 & 205.6 & 196.6 & 188.8 & 181.9 & 175.7 & 170.2 & 165.5 & \\
\hline \multirow{7}{*}{$\begin{array}{c}90 \\
(180)\end{array}$} & C & 12321 & 14075 & 15963 & 18137 & 20436 & 22988 & 25757 & 28750 & & \\
\hline & $P$ & 1911 & 1948 & 1984 & 2018 & 2041 & 2063 & 2081 & 2100 & & \\
\hline & A & 9.86 & 10.00 & 10.15 & 10.27 & 10.35 & 10.44 & 10.52 & 10.59 & & \\
\hline & $M$ & 146.0 & 165.3 & 185.9 & 209.4 & 234.0 & 261.1 & 290.3 & 322.0 & & \\
\hline & $E$ & 6.45 & 7.23 & 8.05 & 8.99 & 10.01 & 11.14 & 12.38 & 13.69 & & \\
\hline & $\%$ & 63.9 & 65.3 & 66.7 & 68.1 & 69.3 & 70.4 & 71.2 & 71.3 & & \\
\hline & $\mathrm{T}$ & 208.0 & 198.4 & 189.7 & 181.5 & 174.1 & 167.7 & 161.9 & 157.8 & & \\
\hline \multirow{7}{*}{$\begin{array}{c}80 \\
(154)\end{array}$} & C & 13051 & 14857 & 16890 & 19114 & 21556 & 24187 & 27110 & 30289 & & \\
\hline & $P$ & 1743 & 1774 & 1802 & 1824 & 1845 & 1854 & 1864 & 1863 & & \\
\hline & A & 9.24 & 9.35 & 9.46 & 9.53 & 9.62 & 9.65 & 9.70 & 9.67 & & \\
\hline & M & 147.6 & 166.7 & 187.9 & 211.0 & 236.1 & 262.8 & 292.3 & 324.2 & & \\
\hline & $\mathrm{E}$ & 7.49 & 8.38 & 9.37 & 10.48 & 11.69 & 13.04 & 14.55 & 16.26 & & \\
\hline & $\%$ & 63.8 & 64.9 & 66.2 & 67.3 & 68.1 & 68.7 & 69.3 & 69.4 & & \\
\hline & $\mathrm{T}$ & 191.2 & 182.5 & 173.9 & 166.2 & 159.5 & 153.5 & 147.9 & 143.2 & & \\
\hline \multirow{7}{*}{$\begin{array}{c}70 \\
(131)\end{array}$} & C & 13819 & 15738 & 17872 & 20238 & & & & & & \\
\hline & $P$ & 1588 & 1614 & 1635 & 1650 & & & & & & \\
\hline & $A$ & 8.67 & 8.76 & 8.83 & 8.89 & & & & & & \\
\hline & $M$ & 149.7 & 169.1 & 190.5 & 214.1 & & & & & & \\
\hline & $\mathrm{E}$ & 8.70 & 9.75 & 10.93 & 12.27 & & & & & & \\
\hline & $\%$ & 62.8 & 63.6 & 64.7 & 65.8 & & & & & & \\
\hline & $\mathrm{T}$ & 176.0 & 167.5 & 159.4 & 151.6 & & & & & & \\
\hline
\end{tabular}

C: Capacity (Btu/hr), P: Power (Watts), A: Current (Amps) at 230V, M: Mass Flow (lbs/hr), E: EER (Btu/Watt-hr) $\%$ : Isentropic Efficiency (\%), T: Discharge Temperature $\left({ }^{\circ} \mathrm{F}\right)$ 
Table A12: L-40 at $40^{\circ} \mathrm{F}$ Superheat, $0^{\circ} \mathrm{F}$ Subcooling

Suction Dew Point Temp, ${ }^{\circ} \mathrm{F}$ (Suction Pressure, psia)

\begin{tabular}{|c|c|c|c|c|c|c|c|c|c|c|c|}
\hline & & $-10(27)$ & $-5(30)$ & $0(34)$ & $5(38)$ & $10(43)$ & $15(47)$ & $20(53)$ & $25(58)$ & $30(64)$ & $35(71)$ \\
\hline \multirow{7}{*}{$\begin{array}{c}140 \\
(362)\end{array}$} & C & & & & & & 18336 & 20714 & 23253 & 26045 & 29030 \\
\hline & P & & & & & & 3448 & 3487 & 3547 & 3590 & 3651 \\
\hline & A & & & & & & 16.01 & 16.19 & 16.42 & 16.63 & 16.89 \\
\hline & $M$ & & & & & & 237.6 & 265.6 & 295.2 & 328.0 & 362.1 \\
\hline & $E$ & & & & & & 5.32 & 5.94 & 6.56 & 7.26 & 7.95 \\
\hline & $\%$ & & & & & & 67.6 & 70.4 & 72.6 & 74.7 & 76.4 \\
\hline & $\mathrm{T}$ & & & & & & 271.9 & 261.2 & 253.1 & 245.5 & 239.7 \\
\hline \multirow{7}{*}{$\begin{array}{c}130 \\
(317)\end{array}$} & C & & & & 15404 & 17498 & 19802 & 22297 & 24883 & 27944 & 31080 \\
\hline & $P$ & & & & 3001 & 3045 & 3087 & 3133 & 3193 & 3232 & 3288 \\
\hline & A & & & & 14.15 & 14.34 & 14.52 & 14.71 & 14.97 & 15.13 & 15.36 \\
\hline & M & & & & 192.1 & 215.9 & 242.3 & 270.3 & 298.8 & 332.9 & 366.8 \\
\hline & $E$ & & & & 5.13 & 5.75 & 6.41 & 7.12 & 7.79 & 8.65 & 9.45 \\
\hline & $\%$ & & & & 66.0 & 68.6 & 71.1 & 73.4 & 74.7 & 77.0 & 78.4 \\
\hline & $\mathrm{T}$ & & & & 271.6 & 260.5 & 250.1 & 241.5 & 235.4 & 227.8 & 223.1 \\
\hline \multirow{7}{*}{$\begin{array}{c}120 \\
(277)\end{array}$} & C & & 12731 & 14548 & 16608 & 18807 & 21174 & 23779 & 26478 & 29681 & 33021 \\
\hline & $P$ & & 2606 & 2649 & 2698 & 2739 & 2784 & 2830 & 2882 & 2910 & 2948 \\
\hline & $A$ & & 12.55 & 12.69 & 12.92 & 13.10 & 13.27 & 13.46 & 13.69 & 13.79 & 13.95 \\
\hline & $M$ & & 153.3 & 173.8 & 196.4 & 220.6 & 246.1 & 273.8 & 302.4 & 336.3 & 371.0 \\
\hline & $E$ & & 4.88 & 5.49 & 6.16 & 6.87 & 7.61 & 8.40 & 9.19 & 10.20 & 11.20 \\
\hline & $\%$ & & 64.0 & 66.4 & 69.2 & 71.5 & 73.5 & 75.4 & 76.7 & 78.4 & 79.7 \\
\hline & $\mathrm{T}$ & & 273.8 & 261.4 & 249.2 & 239.3 & 231.3 & 224.0 & 218.0 & 211.8 & 207.2 \\
\hline \multirow{7}{*}{$\begin{array}{c}110 \\
(241)\end{array}$} & C & & 13723 & 15735 & 17696 & 19973 & 22469 & 25194 & 27892 & 31427 & 34921 \\
\hline & $\mathrm{P}$ & & 2357 & 2394 & 2434 & 2481 & 2522 & 2559 & 2595 & 2618 & 2641 \\
\hline & A & & 11.53 & 11.68 & 11.87 & 12.06 & 12.22 & 12.37 & 12.53 & 12.61 & 12.71 \\
\hline & $M$ & & 157.3 & 178.7 & 199.5 & 223.0 & 248.9 & 276.8 & 303.9 & 339.6 & 374.5 \\
\hline & $E$ & & 5.82 & 6.57 & 7.27 & 8.05 & 8.91 & 9.85 & 10.75 & 12.01 & 13.22 \\
\hline & $\%$ & & 67.5 & 71.2 & 71.2 & 72.8 & 74.5 & 76.2 & 76.8 & 78.9 & 80.0 \\
\hline & $\mathrm{T}$ & & 249.0 & 235.6 & 230.1 & 222.3 & 214.9 & 208.2 & 203.4 & 197.0 & 192.4 \\
\hline \multirow{8}{*}{$\begin{array}{c}100 \\
(209)\end{array}$} & $C$ & 12733 & 14568 & 16544 & 18713 & 21076 & 23703 & 26543 & 29402 & 33022 & \\
\hline & $P$ & 2089 & 2127 & 2171 & 2211 & 2246 & 2279 & 2306 & 2332 & 2353 & \\
\hline & $A$ & 10.52 & 10.68 & 10.84 & 11.00 & 11.14 & 11.28 & 11.38 & 11.49 & 11.57 & \\
\hline & $M$ & 140.7 & 159.5 & 179.7 & 201.6 & 225.3 & 251.3 & 279.1 & 306.7 & 341.9 & \\
\hline & $\mathrm{E}$ & 6.10 & 6.85 & 7.62 & 8.46 & 9.39 & 10.40 & 11.51 & 12.61 & 14.03 & \\
\hline & $\%$ & 66.5 & 69.0 & 70.6 & 72.2 & 73.8 & 75.1 & 76.3 & 76.8 & 77.8 & \\
\hline & $\mathrm{T}$ & 242.0 & 230.1 & 221.1 & 212.8 & 205.3 & 198.7 & 192.8 & 188.3 & 183.7 & \\
\hline & C & 13512 & 15362 & 17410 & 19696 & 22184 & 24899 & 27816 & 30707 & & \\
\hline \multirow{6}{*}{$\begin{array}{c}90 \\
(180)\end{array}$} & $P$ & 1903 & 1939 & 1974 & 2004 & 2033 & 2054 & 2076 & 2094 & & \\
\hline & A & 9.85 & 9.96 & 10.10 & 10.21 & 10.33 & 10.41 & 10.49 & 10.56 & & \\
\hline & $M$ & 143.0 & 161.3 & 181.4 & 203.6 & 227.4 & 253.3 & 280.8 & 307.5 & & \\
\hline & $E$ & 7.10 & 7.92 & 8.82 & 9.83 & 10.91 & 12.12 & 13.40 & 14.66 & & \\
\hline & $\%$ & 68.3 & 69.5 & 71.0 & 72.5 & 73.6 & 74.5 & 74.8 & 73.4 & & \\
\hline & $\mathrm{T}$ & 221.7 & 213.0 & 204.6 & 196.8 & 190.1 & 184.2 & 180.0 & 177.2 & & \\
\hline \multirow{7}{*}{$\begin{array}{c}80 \\
(154)\end{array}$} & $\mathrm{C}$ & 14192 & 16123 & 18257 & 20617 & 23189 & 26021 & 29078 & 32295 & & \\
\hline & $P$ & 1735 & 1764 & 1790 & 1812 & 1832 & 1845 & 1857 & 1858 & & \\
\hline & A & 9.20 & 9.31 & 9.41 & 9.50 & 9.56 & 9.60 & 9.65 & 9.66 & & \\
\hline & $M$ & 144.2 & 162.7 & 182.8 & 204.8 & 228.7 & 254.6 & 282.6 & 311.4 & & \\
\hline & $E$ & 8.18 & 9.14 & 10.20 & 11.38 & 12.66 & 14.11 & 15.66 & 17.38 & & \\
\hline & $\%$ & 67.9 & 69.3 & 70.5 & 71.6 & 72.3 & 72.7 & 72.5 & 72.6 & & \\
\hline & $\mathrm{T}$ & 206.2 & 197.0 & 189.1 & 182.0 & 176.0 & 170.5 & 166.4 & 161.6 & & \\
\hline \multirow{7}{*}{$\begin{array}{c}70 \\
(131)\end{array}$} & C & 14410 & 16370 & 18540 & 20917 & & & & & & \\
\hline & $P$ & 1576 & 1600 & 1621 & 1635 & & & & & & \\
\hline & $A$ & 8.63 & 8.72 & 8.79 & 8.83 & & & & & & \\
\hline & $M$ & 145.2 & 163.6 & 183.9 & 205.8 & & & & & & \\
\hline & $\mathrm{E}$ & 9.14 & 10.23 & 11.44 & 12.79 & & & & & & \\
\hline & $\%$ & 67.1 & 67.9 & 68.7 & 69.4 & & & & & & \\
\hline & $\mathrm{T}$ & 190.5 & 182.7 & 175.5 & 168.8 & & & & & & \\
\hline
\end{tabular}

C: Capacity (Btu/hr), P: Power (Watts), A: Current (Amps) at 230V, M: Mass Flow (lbs/hr), E: EER (Btu/Watt-hr) $\%$ : Isentropic Efficiency (\%), T: Discharge Temperature $\left({ }^{\circ} \mathrm{F}\right)$ 
Table A13: R-32 + R-134a mixture at $65^{\circ} \mathrm{F}$ Suction Temperature, $0^{\circ} \mathrm{F}$ Subcooling

Suction Dew Point Temp, ${ }^{\circ} \mathrm{F}$ (Suction Pressure, psia)

\begin{tabular}{|c|c|c|c|c|c|c|c|c|c|c|c|}
\hline & & $-10(31)$ & $-5(34)$ & 0 (39) & $5(43)$ & $10(48)$ & $15(54)$ & 20 (59) & $25(66)$ & $30(73)$ & $35(80)$ \\
\hline \multirow{7}{*}{$\begin{array}{c}140 \\
(410)\end{array}$} & C & & & & & & & & & 25559 & 28589 \\
\hline & $P$ & & & & & & & & & 4224 & 4268 \\
\hline & A & & & & & & & & & 19.34 & 19.54 \\
\hline & $M$ & & & & & & & & & 328.6 & 369.9 \\
\hline & $E$ & & & & & & & & & 6.05 & 6.70 \\
\hline & $\%$ & & & & & & & & & 68.0 & 70.0 \\
\hline & $\mathrm{T}$ & & & & & & & & & 271.4 & 256.2 \\
\hline \multirow{7}{*}{$\begin{array}{c}130 \\
(360)\end{array}$} & $C$ & & & & & & & & 25057 & 28040 & 31186 \\
\hline & $\mathrm{P}$ & & & & & & & & 3712 & 3751 & 3803 \\
\hline & A & & & & & & & & 17.14 & 17.30 & 17.53 \\
\hline & M & & & & & & & & 301.2 & 339.1 & 380.1 \\
\hline & $\mathrm{E}$ & & & & & & & & 6.75 & 7.48 & 8.20 \\
\hline & $\%$ & & & & & & & & 70.5 & 72.0 & 73.5 \\
\hline & $\mathrm{T}$ & & & & & & & & 261.6 & 247.6 & 234.9 \\
\hline \multirow{7}{*}{$\begin{array}{c}120 \\
(314)\end{array}$} & C & & & & & & 21433 & 24070 & 26994 & 30136 & 33613 \\
\hline & $P$ & & & & & & 3229 & 3266 & 3302 & 3337 & 3366 \\
\hline & A & & & & & & 15.08 & 15.25 & 15.40 & 15.55 & 15.67 \\
\hline & $M$ & & & & & & 241.7 & 272.6 & 307.3 & 345.0 & 387.1 \\
\hline & $E$ & & & & & & 6.64 & 7.37 & 8.17 & 9.03 & 9.99 \\
\hline & $\%$ & & & & & & 70.6 & 71.9 & 73.4 & 74.4 & 76.0 \\
\hline & T & & & & & & 268.5 & 254.1 & 240.2 & 227.6 & 214.7 \\
\hline \multirow{7}{*}{$\begin{array}{c}110 \\
(273)\end{array}$} & C & & & & & 20137 & 22760 & 25633 & 28674 & 31944 & \\
\hline & $\mathrm{P}$ & & & & & 2851 & 2880 & 2915 & 2945 & 2975 & \\
\hline & A & & & & & 13.50 & 13.63 & 13.78 & 13.90 & 14.03 & \\
\hline & $\mathrm{M}$ & & & & & 215.3 & 244.3 & 276.4 & 310.8 & 348.1 & \\
\hline & $E$ & & & & & 7.06 & 7.90 & 8.79 & 9.74 & 10.74 & \\
\hline & $\%$ & & & & & 71.0 & 72.6 & 73.9 & 74.6 & 75.2 & \\
\hline & $\mathrm{T}$ & & & & & 263.0 & 247.8 & 233.9 & 221.9 & 210.3 & \\
\hline \multirow{7}{*}{$\begin{array}{c}100 \\
(237)\end{array}$} & C & & & 16589 & 18958 & 21491 & 24186 & 27143 & 30397 & 33944 & \\
\hline & $P$ & & & 2480 & 2517 & 2545 & 2572 & 2606 & 2631 & 2656 & \\
\hline & A & & & 12.02 & 12.18 & 12.28 & 12.40 & 12.53 & 12.64 & 12.74 & \\
\hline & $M$ & & & 168.4 & 193.1 & 219.6 & 248.1 & 279.6 & 314.6 & 353.5 & \\
\hline & $\mathrm{E}$ & & & 6.69 & 7.53 & 8.44 & 9.41 & 10.42 & 11.55 & 12.78 & \\
\hline & $\%$ & & & 70.4 & 71.9 & 73.4 & 74.3 & 74.9 & 75.5 & 76.8 & \\
\hline & T & & & 272.5 & 256.3 & 241.1 & 227.9 & 215.7 & 203.9 & 191.9 & \\
\hline \multirow{7}{*}{$\begin{array}{c}90 \\
(204)\end{array}$} & C & & 15614 & 17825 & 20237 & 22835 & 25656 & 28693 & 31809 & & \\
\hline & $\mathrm{P}$ & & 2187 & 2213 & 2245 & 2273 & 2301 & 2323 & 2341 & & \\
\hline & A & & 10.92 & 11.01 & 11.14 & 11.23 & 11.36 & 11.44 & 11.52 & & \\
\hline & $M$ & & 151.6 & 173.6 & 197.7 & 223.8 & 252.3 & 283.3 & 315.9 & & \\
\hline & E & & 7.14 & 8.05 & 9.01 & 10.05 & 11.15 & 12.35 & 13.59 & & \\
\hline & $\%$ & & 72.0 & 73.5 & 74.4 & 74.9 & 75.1 & 74.7 & 72.6 & & \\
\hline & T & & 263.8 & 248.3 & 234.1 & 221.4 & 209.5 & 198.8 & 190.8 & & \\
\hline \multirow{7}{*}{$\begin{array}{c}80 \\
(175)\end{array}$} & C & 14540 & 16659 & 18903 & 21343 & 24024 & 26914 & 30124 & & & \\
\hline & $\mathrm{P}$ & 1933 & 1956 & 1988 & 2016 & 2037 & 2051 & 2061 & & & \\
\hline & A & 9.93 & 10.01 & 10.14 & 10.25 & 10.34 & 10.38 & 10.43 & & & \\
\hline & $M$ & 135.5 & 155.6 & 177.0 & 200.4 & 226.3 & 254.4 & 286.0 & & & \\
\hline & $E$ & 7.52 & 8.52 & 9.51 & 10.59 & 11.80 & 13.12 & 14.61 & & & \\
\hline & $\%$ & 73.2 & 74.4 & 74.6 & 74.8 & 74.7 & 74.0 & 73.8 & & & \\
\hline & $\mathrm{T}$ & 256.5 & 241.2 & 228.1 & 215.8 & 204.0 & 193.4 & 182.5 & & & \\
\hline \multirow{7}{*}{$\begin{array}{c}70 \\
(149)\end{array}$} & C & 15573 & 17618 & 19933 & 22529 & & & & & & \\
\hline & $\mathrm{P}$ & 1740 & 1765 & 1784 & 1802 & & & & & & \\
\hline & A & 9.22 & 9.33 & 9.38 & 9.44 & & & & & & \\
\hline & $M$ & 140.0 & 158.7 & 180.0 & 204.0 & & & & & & \\
\hline & $E$ & 8.95 & 9.98 & 11.17 & 12.50 & & & & & & \\
\hline & $\%$ & 74.3 & 74.0 & 73.9 & 74.8 & & & & & & \\
\hline & $\mathrm{T}$ & 235.9 & 223.6 & 211.2 & 197.9 & & & & & & \\
\hline
\end{tabular}

C: Capacity (Btu/hr), P: Power (Watts), A: Current (Amps) at 230V, M: Mass Flow (lbs/hr), E: EER (Btu/Watt-hr) $\%$ : Isentropic Efficiency (\%), T: Discharge Temperature $\left({ }^{\circ} \mathrm{F}\right)$ 
Table A14: R-32 + R-134a mixture at $20^{\circ} \mathrm{F}$ Superheat, $0^{\circ} \mathrm{F}$ Subcooling

Suction Dew Point Temp, ${ }^{\circ} \mathrm{F}$ (Suction Pressure, psia)

\begin{tabular}{|c|c|c|c|c|c|c|c|c|c|c|c|}
\hline & & $-10(31)$ & $-5(34)$ & 0 (39) & 5 (43.) & $10(48)$. & 15 (54.) & 20 (59.) & 25 (66.) & 30 (73.) & 35 (80.) \\
\hline \multirow{7}{*}{$\begin{array}{c}140 \\
(410)\end{array}$} & C & & & & & & & & 22301 & 25315 & 28647 \\
\hline & $P$ & & & & & & & & 4178 & 4231 & 4260 \\
\hline & A & & & & & & & & 19.13 & 19.38 & 19.51 \\
\hline & M & & & & & & & & 302.4 & 340.6 & 382.2 \\
\hline & $\mathrm{E}$ & & & & & & & & 5.34 & 5.98 & 6.72 \\
\hline & $\%$ & & & & & & & & 63.8 & 66.6 & 71.0 \\
\hline & $\mathrm{T}$ & & & & & & & & 268.6 & 256.2 & 242.9 \\
\hline \multirow{7}{*}{$\begin{array}{c}130 \\
(360)\end{array}$} & C & & & & & & 19282 & 21924 & 24788 & 27878 & 31379 \\
\hline & $P$ & & & & & & 3602 & 3650 & 3688 & 3732 & 3776 \\
\hline & A & & & & & & 16.67 & 16.89 & 17.06 & 17.25 & 17.44 \\
\hline & M & & & & & & 249.2 & 281.0 & 315.3 & 352.1 & 393.7 \\
\hline & $\mathrm{E}$ & & & & & & 5.35 & 6.01 & 6.72 & 7.47 & 8.31 \\
\hline & $\%$ & & & & & & 63.3 & 66.0 & 68.5 & 70.9 & 75.1 \\
\hline & $\mathrm{T}$ & & & & & & 265.9 & 253.1 & 241.9 & 232.0 & 221.1 \\
\hline \multirow{7}{*}{$\begin{array}{c}120 \\
(314)\end{array}$} & $C$ & & & & 15982 & 18391 & 20952 & 23733 & 26676 & 29876 & 33309 \\
\hline & $P$ & & & & 3118 & 3160 & 3208 & 3249 & 3288 & 3327 & 3369 \\
\hline & A & & & & 14.64 & 14.82 & 15.02 & 15.19 & 15.36 & 15.53 & 15.71 \\
\hline & M & & & & 197.9 & 225.9 & 255.4 & 287.2 & 320.4 & 356.3 & 395.0 \\
\hline & $\mathrm{E}$ & & & & 5.13 & 5.82 & 6.53 & 7.31 & 8.11 & 8.98 & 9.89 \\
\hline & $\%$ & & & & 61.1 & 64.0 & 66.7 & 68.9 & 70.9 & 72.4 & 74.2 \\
\hline & $\mathrm{T}$ & & & & 269.1 & 254.0 & 241.3 & 230.3 & 221.2 & 213.5 & 206.4 \\
\hline \multirow{7}{*}{$\begin{array}{c}110 \\
(273)\end{array}$} & $C$ & & & 15164 & 17437 & 19892 & 22520 & 25366 & 28480 & 31913 & \\
\hline & $P$ & & & 2755 & 2803 & 2844 & 2879 & 2913 & 2947 & 2971 & \\
\hline & A & & & 13.16 & 13.36 & 13.52 & 13.68 & 13.80 & 13.95 & 14.05 & \\
\hline & M & & & 179.2 & 204.5 & 231.6 & 260.3 & 291.1 & 324.7 & 361.4 & \\
\hline & $E$ & & & 5.50 & 6.22 & 6.99 & 7.82 & 8.71 & 9.66 & 10.74 & \\
\hline & $\%$ & & & 61.6 & 64.2 & 66.5 & 68.6 & 70.2 & 71.8 & 74.0 & \\
\hline & $\mathrm{T}$ & & & 258.3 & 243.5 & 231.1 & 220.5 & 211.6 & 203.5 & 195.0 & \\
\hline \multirow{7}{*}{$\begin{array}{c}100 \\
(237)\end{array}$} & C & & & 16475 & 18777 & 21317 & 24063 & 27018 & 30272 & 33817 & \\
\hline & $P$ & & & 2476 & 2509 & 2542 & 2578 & 2607 & 2633 & 2651 & \\
\hline & A & & & 12.04 & 12.17 & 12.30 & 12.44 & 12.57 & 12.67 & 12.75 & \\
\hline & M & & & 185.3 & 209.6 & 236.3 & 264.9 & 295.4 & 328.9 & 365.3 & \\
\hline & $\mathrm{E}$ & & & 6.65 & 7.48 & 8.39 & 9.33 & 10.36 & 11.50 & 12.76 & \\
\hline & $\%$ & & & 64.4 & 66.7 & 68.6 & 70.0 & 71.1 & 72.2 & 73.7 & \\
\hline & $\mathrm{T}$ & & & 232.6 & 220.0 & 209.4 & 200.6 & 192.8 & 185.6 & 178.7 & \\
\hline \multirow{7}{*}{$\begin{array}{c}90 \\
(204)\end{array}$} & C & & 15404 & 17620 & 20024 & 22635 & 25464 & 28550 & 31847 & & \\
\hline & $P$ & & 2185 & 2221 & 2258 & 2286 & 2311 & 2328 & 2347 & & \\
\hline & A & & 10.91 & 11.04 & 11.19 & 11.30 & 11.39 & 11.46 & 11.54 & & \\
\hline & M & & 166.6 & 189.2 & 213.5 & 239.7 & 267.9 & 298.5 & 331.2 & & \\
\hline & $\mathrm{E}$ & & 7.05 & 7.93 & 8.87 & 9.90 & 11.02 & 12.27 & 13.57 & & \\
\hline & $\%$ & & 64.3 & 66.2 & 67.7 & 68.9 & 69.8 & 70.5 & 70.6 & & \\
\hline & $\mathrm{T}$ & & 222.2 & 210.3 & 200.0 & 191.1 & 183.3 & 176.2 & 170.9 & & \\
\hline \multirow{7}{*}{$\begin{array}{c}80 \\
(175)\end{array}$} & C & 14284 & 16396 & 18673 & 21120 & 23848 & 26843 & 30164 & 33663 & & \\
\hline & $P$ & 1938 & 1975 & 2006 & 2032 & 2052 & 2064 & 2074 & 2071 & & \\
\hline & A & 9.95 & 10.10 & 10.21 & 10.32 & 10.40 & 10.45 & 10.51 & 10.48 & & \\
\hline & M & 149.0 & 169.8 & 192.1 & 215.8 & 242.1 & 270.7 & 302.5 & 335.5 & & \\
\hline & $E$ & 7.37 & 8.30 & 9.31 & 10.40 & 11.62 & 13.00 & 14.54 & 16.26 & & \\
\hline & $\%$ & 63.7 & 65.1 & 66.2 & 67.3 & 68.1 & 68.6 & 69.3 & 69.2 & & \\
\hline & $\mathrm{T}$ & 213.1 & 201.5 & 191.4 & 182.4 & 174.2 & 166.8 & 160.0 & 154.0 & & \\
\hline \multirow{7}{*}{$\begin{array}{c}70 \\
(149)\end{array}$} & C & 15238 & 17357 & 19699 & 22213 & & & & & & \\
\hline & $P$ & 1755 & 1779 & 1803 & 1819 & & & & & & \\
\hline & A & 9.28 & 9.36 & 9.47 & 9.53 & & & & & & \\
\hline & $\mathrm{M}$ & 152.5 & 172.6 & 194.6 & 218.1 & & & & & & \\
\hline & $\mathrm{E}$ & 8.68 & 9.76 & 10.92 & 12.21 & & & & & & \\
\hline & $\%$ & 63.4 & 64.3 & 65.0 & 64.6 & & & & & & \\
\hline & $\mathrm{T}$ & 194.3 & 184.0 & 174.8 & 167.9 & & & & & & \\
\hline
\end{tabular}

C: Capacity (Btu/hr), P: Power (Watts), A: Current (Amps) at 230V, M: Mass Flow (lbs/hr), E: EER (Btu/Watt-hr) $\%$ : Isentropic Efficiency (\%), T: Discharge Temperature $\left({ }^{\circ} \mathrm{F}\right)$ 
Table A15: R-32 + R-134a mixture at $40^{\circ} \mathrm{F}$ Superheat, $0^{\circ} \mathrm{F}$ Subcooling

Suction Dew Point Temp, ${ }^{\circ} \mathrm{F}$ (Suction Pressure, psia)

\begin{tabular}{|c|c|c|c|c|c|c|c|c|c|c|c|}
\hline & & $-10(31)$ & $-5(34)$ & $0(39)$ & $5(43)$ & $10(48)$ & $15(54)$ & $20(59)$ & $25(66)$ & $30(73)$ & $35(80)$ \\
\hline \multirow{7}{*}{$\begin{array}{c}140 \\
(410)\end{array}$} & C & & & & & & & & & 28072 & 31564 \\
\hline & P & & & & & & & & & 4203 & 4256 \\
\hline & A & & & & & & & & & 19.23 & 19.47 \\
\hline & M & & & & & & & & & 326.6 & 364.4 \\
\hline & $\mathrm{E}$ & & & & & & & & & 6.68 & 7.42 \\
\hline & $\%$ & & & & & & & & & 69.2 & 72.2 \\
\hline & $\mathrm{T}$ & & & & & & & & & 274.6 & 263.7 \\
\hline \multirow{7}{*}{$\begin{array}{c}130 \\
(360)\end{array}$} & C & & & & & & & 24177 & 27069 & 30498 & 34062 \\
\hline & P & & & & & & & 3638 & 3712 & 3730 & 3780 \\
\hline & A & & & & & & & 16.82 & 17.14 & 17.21 & 17.42 \\
\hline & M & & & & & & & 271.3 & 301.2 & 337.1 & 373.7 \\
\hline & $\mathrm{E}$ & & & & & & & 6.65 & 7.29 & 8.18 & 9.01 \\
\hline & $\%$ & & & & & & & 69.1 & 70.5 & 73.0 & 74.7 \\
\hline & $\mathrm{T}$ & & & & & & & 270.2 & 261.6 & 251.4 & 243.9 \\
\hline \multirow{7}{*}{$\begin{array}{c}120 \\
(314)\end{array}$} & C & & & & & 20156 & 22943 & 25887 & 28973 & 32400 & 36054 \\
\hline & $P$ & & & & & 3151 & 3203 & 3241 & 3302 & 3323 & 3360 \\
\hline & A & & & & & 14.78 & 14.99 & 15.16 & 15.40 & 15.51 & 15.67 \\
\hline & $M$ & & & & & 218.4 & 247.0 & 276.5 & 307.3 & 341.4 & 377.3 \\
\hline & $\mathrm{E}$ & & & & & 6.40 & 7.16 & 7.99 & 8.77 & 9.75 & 10.73 \\
\hline & $\%$ & & & & & 67.2 & 69.7 & 71.9 & 73.4 & 75.0 & 76.3 \\
\hline & $\mathrm{T}$ & & & & & 270.8 & 258.6 & 248.0 & 240.2 & 232.2 & 225.7 \\
\hline \multirow{7}{*}{$\begin{array}{c}110 \\
(273)\end{array}$} & $C$ & & & 16653 & 19101 & 21670 & 24461 & 27465 & 30604 & 34277 & \\
\hline & $P$ & & & 2742 & 2789 & 2830 & 2868 & 2906 & 2945 & 2967 & \\
\hline & A & & & 13.10 & 13.30 & 13.46 & 13.62 & 13.78 & 13.90 & 14.03 & \\
\hline & $M$ & & & 175.2 & 199.4 & 224.5 & 251.8 & 280.7 & 310.8 & 345.7 & \\
\hline & $\mathrm{E}$ & & & 6.07 & 6.85 & 7.66 & 8.53 & 9.45 & 10.39 & 11.55 & \\
\hline & $\%$ & & & 65.2 & 68.0 & 70.0 & 71.9 & 73.4 & 74.6 & 76.1 & \\
\hline & $\mathrm{T}$ & & & 272.7 & 258.7 & 247.5 & 237.5 & 229.2 & 221.9 & 214.6 & \\
\hline \multirow{7}{*}{$\begin{array}{c}100 \\
(237)\end{array}$} & $C$ & & & 17899 & 20346 & 22990 & 25867 & 28999 & 32300 & 36063 & \\
\hline & $P$ & & & 2461 & 2501 & 2535 & 2569 & 2597 & 2631 & 2648 & \\
\hline & $A$ & & & 11.98 & 12.15 & 12.28 & 12.42 & 12.53 & 12.64 & 12.74 & \\
\hline & M & & & 180.4 & 203.7 & 228.4 & 255.3 & 284.4 & 314.6 & 349.1 & \\
\hline & $\mathrm{E}$ & & & 7.27 & 8.14 & 9.07 & 10.07 & 11.17 & 12.28 & 13.62 & \\
\hline & $\%$ & & & 68.4 & 70.4 & 71.8 & 73.2 & 74.4 & 75.5 & 76.0 & \\
\hline & $\mathrm{T}$ & & & 247.3 & 236.0 & 226.7 & 218.3 & 210.5 & 203.9 & 198.6 & \\
\hline \multirow{7}{*}{$\begin{array}{c}90 \\
(204)\end{array}$} & C & & 16727 & 19025 & 21478 & 24213 & 27218 & 30402 & 33703 & & \\
\hline & $P$ & & 2179 & 2217 & 2246 & 2276 & 2304 & 2328 & 2341 & & \\
\hline & A & & 10.88 & 11.04 & 11.13 & 11.26 & 11.37 & 11.47 & 11.52 & & \\
\hline & M & & 163.0 & 184.2 & 206.6 & 231.2 & 258.3 & 286.7 & 315.9 & & \\
\hline & $\mathrm{E}$ & & 7.68 & 8.58 & 9.56 & 10.64 & 11.81 & 13.06 & 14.40 & & \\
\hline & $\%$ & & 68.2 & 70.1 & 71.2 & 72.2 & 73.1 & 73.2 & 72.6 & & \\
\hline & $\mathrm{T}$ & & 237.2 & 225.8 & 216.8 & 208.7 & 201.2 & 195.8 & 190.8 & & \\
\hline \multirow{7}{*}{$\begin{array}{c}80 \\
(175)\end{array}$} & $C$ & 15458 & 17619 & 19931 & 22521 & 25375 & 28545 & 31847 & 35586 & & \\
\hline & $P$ & 1931 & 1970 & 1996 & 2018 & 2038 & 2056 & 2061 & 2065 & & \\
\hline & A & 9.93 & 10.09 & 10.19 & 10.27 & 10.35 & 10.42 & 10.43 & 10.46 & & \\
\hline & M & 146.1 & 165.5 & 185.8 & 208.5 & 233.5 & 261.1 & 289.2 & 321.8 & & \\
\hline & $\mathrm{E}$ & 8.00 & 8.95 & 9.98 & 11.16 & 12.45 & 13.88 & 15.45 & 17.23 & & \\
\hline & $\%$ & 68.0 & 69.1 & 70.2 & 70.9 & 71.6 & 72.2 & 72.5 & 71.5 & & \\
\hline & $\mathrm{T}$ & 226.9 & 216.8 & 207.5 & 199.3 & 191.6 & 184.4 & 178.5 & 173.8 & & \\
\hline \multirow{7}{*}{$\begin{array}{c}70 \\
(149)\end{array}$} & C & 16001 & 18188 & 20603 & 23270 & & & & & & \\
\hline & P & 1745 & 1767 & 1790 & 1808 & & & & & & \\
\hline & $A$ & 9.24 & 9.32 & 9.40 & 9.48 & & & & & & \\
\hline & $M$ & 148.0 & 167.2 & 188.2 & 211.2 & & & & & & \\
\hline & $\mathrm{E}$ & 9.17 & 10.29 & 11.51 & 12.87 & & & & & & \\
\hline & $\%$ & 67.4 & 68.3 & 69.0 & 69.4 & & & & & & \\
\hline & $\mathrm{T}$ & 209.4 & 199.7 & 191.0 & 183.2 & & & & & & \\
\hline
\end{tabular}

C: Capacity (Btu/hr), P: Power (Watts), A: Current (Amps) at 230V, M: Mass Flow (lbs/hr), E: EER (Btu/Watt-hr) $\%$ : Isentropic Efficiency (\%), T: Discharge Temperature $\left({ }^{\circ} \mathrm{F}\right)$ 


\section{Appendix B}

\section{$\underline{\text { Performance Maps }}$}

\section{- Capacity, Input Power, and EER}

Figures B1 to B20 present measured capacity, input power, EER, and isentropic efficiency as a function of suction dew point temperature for given discharge dew point temperature at $65^{\circ} \mathrm{F}$ suction temperature and $0^{\circ} \mathrm{F}$ subcooling for baseline and each alternative refrigerant tested. Note that some irregular variations in isentropic efficiency are observed, particularly in Figures B19 and B20. This is due to small variations in discharge pressure, which is within the specified tolerance for the calorimeter. Steady state conditions for saturated suction temperature, suction temperature, saturated discharge temperature, and expansion valve inlet temperature were specified as setpoint temperature $\pm 0.5^{\circ} \mathrm{F}$.

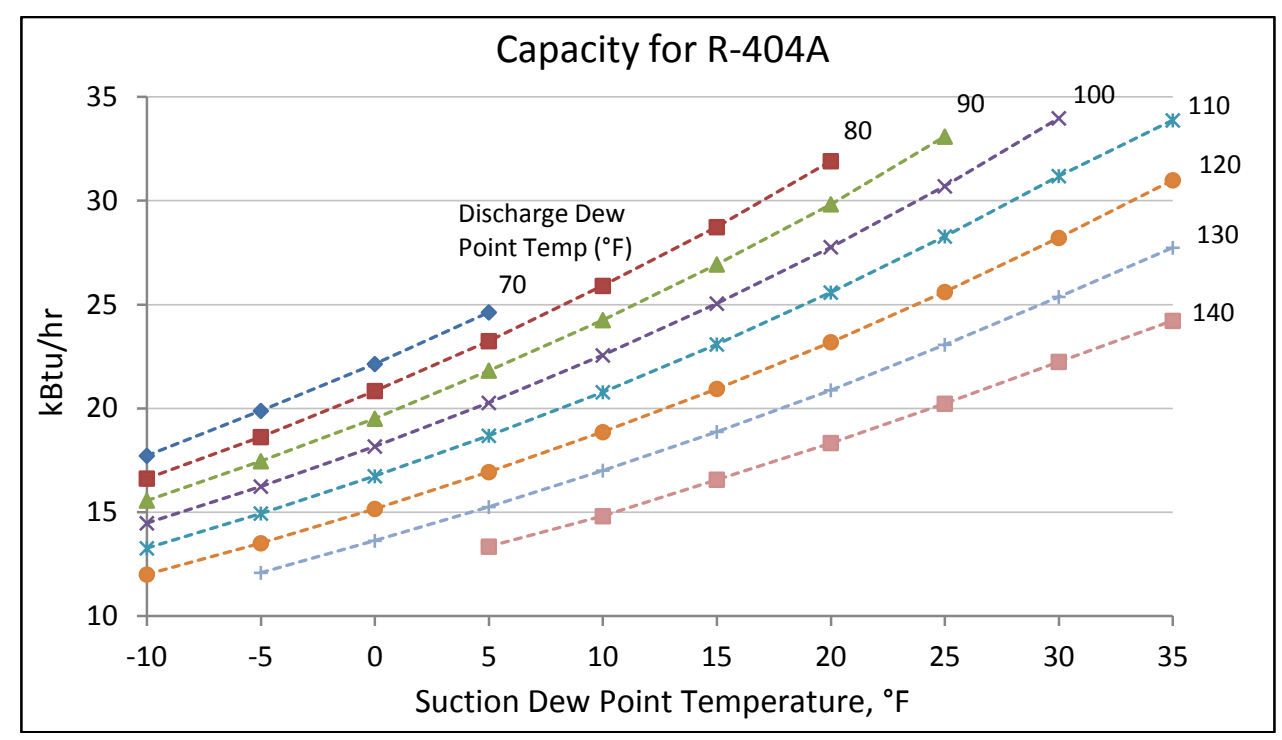

Figure B1 Capacity for R-404A. 


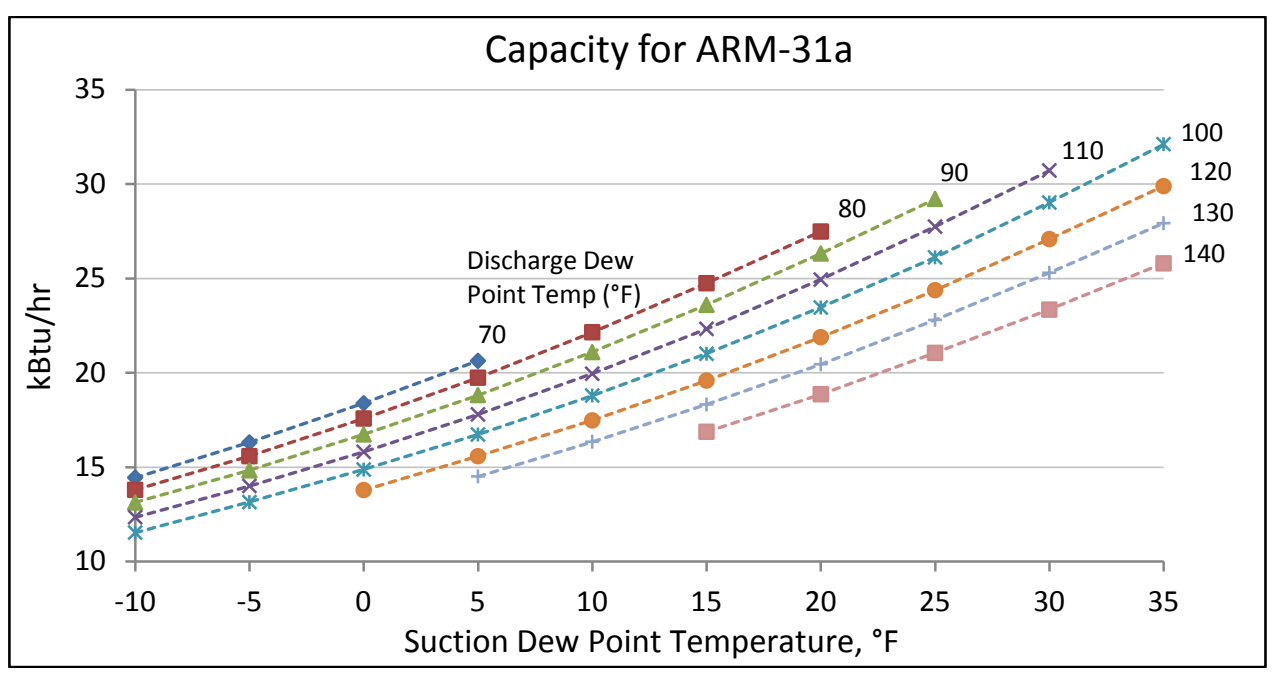

Figure B2 Capacity for ARM-31a.

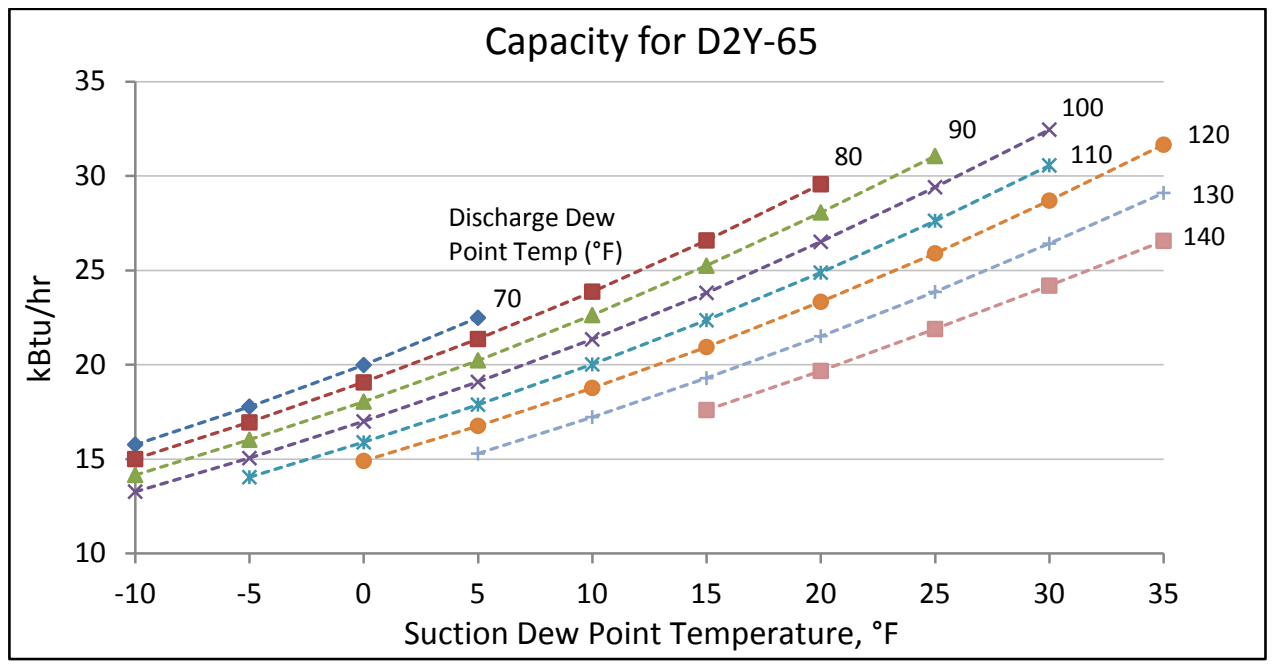

Figure B3 Capacity for D2Y-65. 


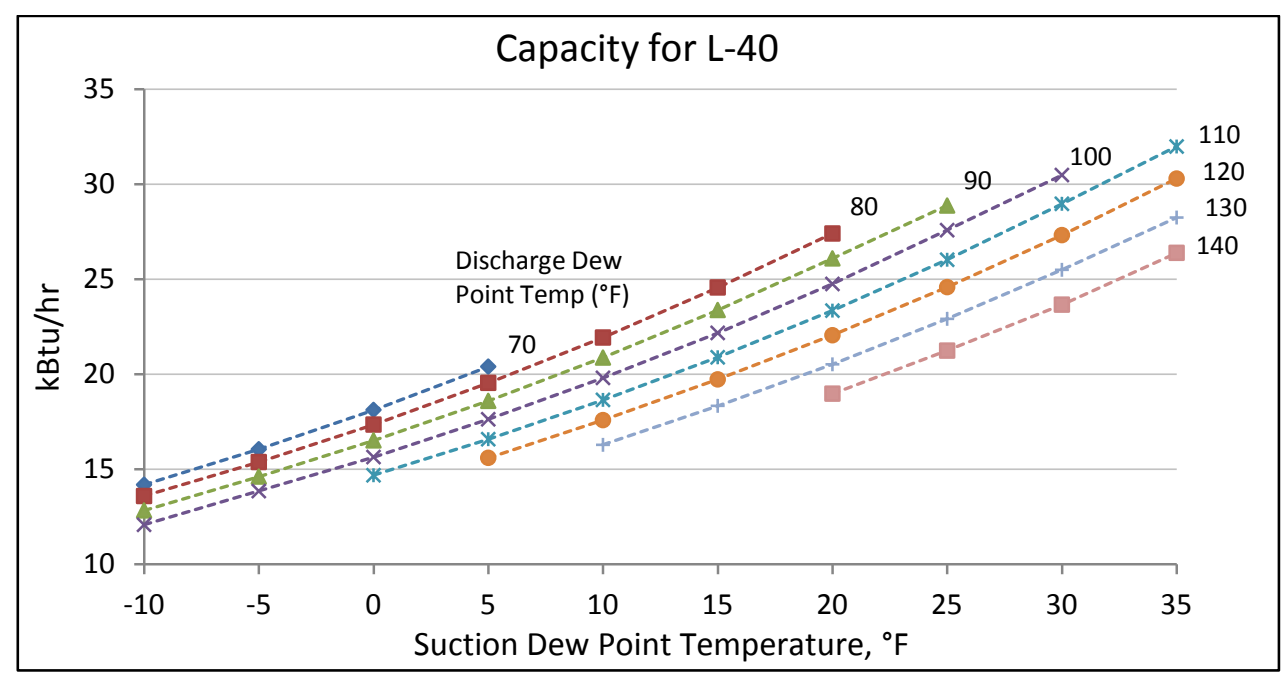

Figure B4 Capacity for L-40.

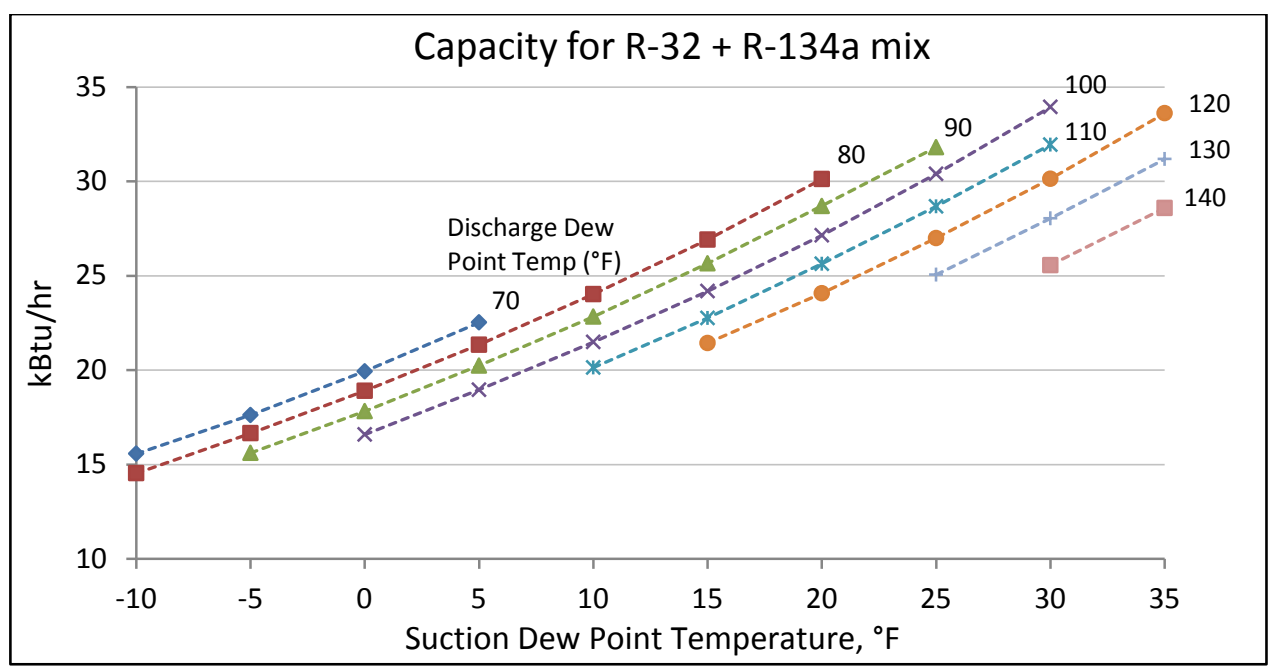

Figure B5 Capacity for R-32 + R-134a mixture. 


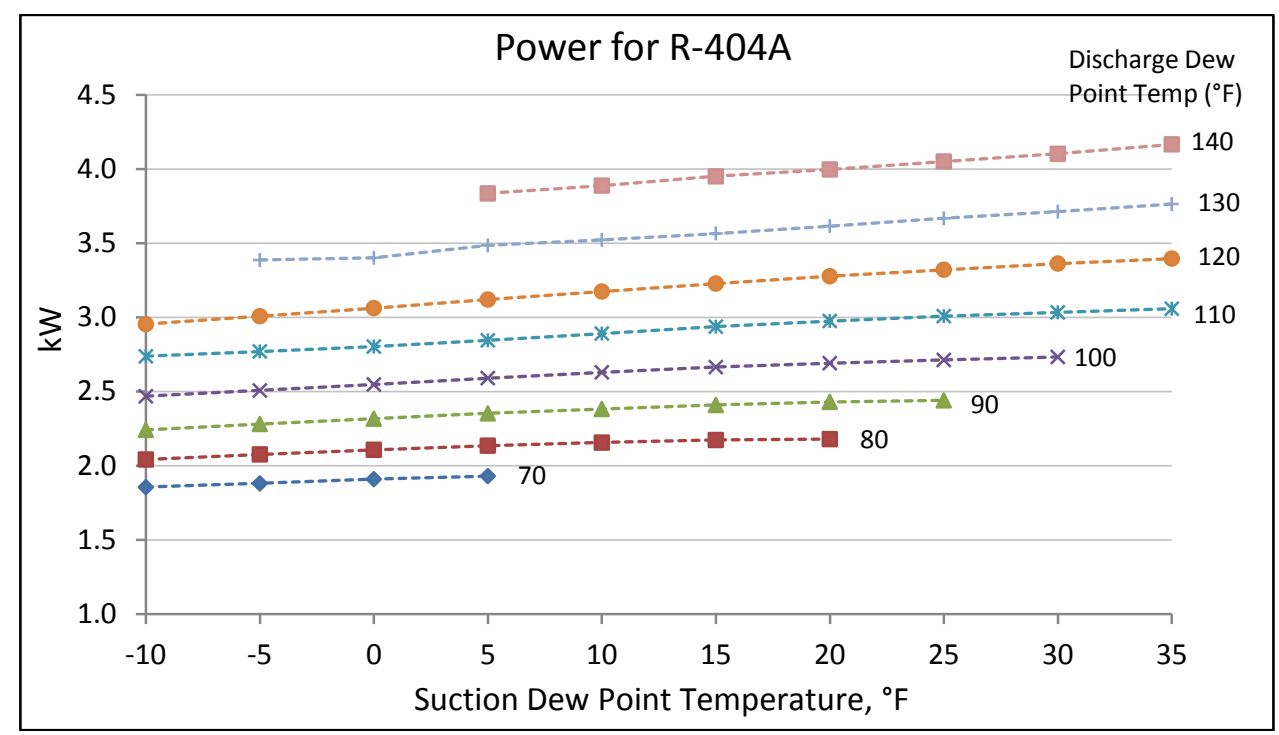

Figure B6 Power for R-404A.

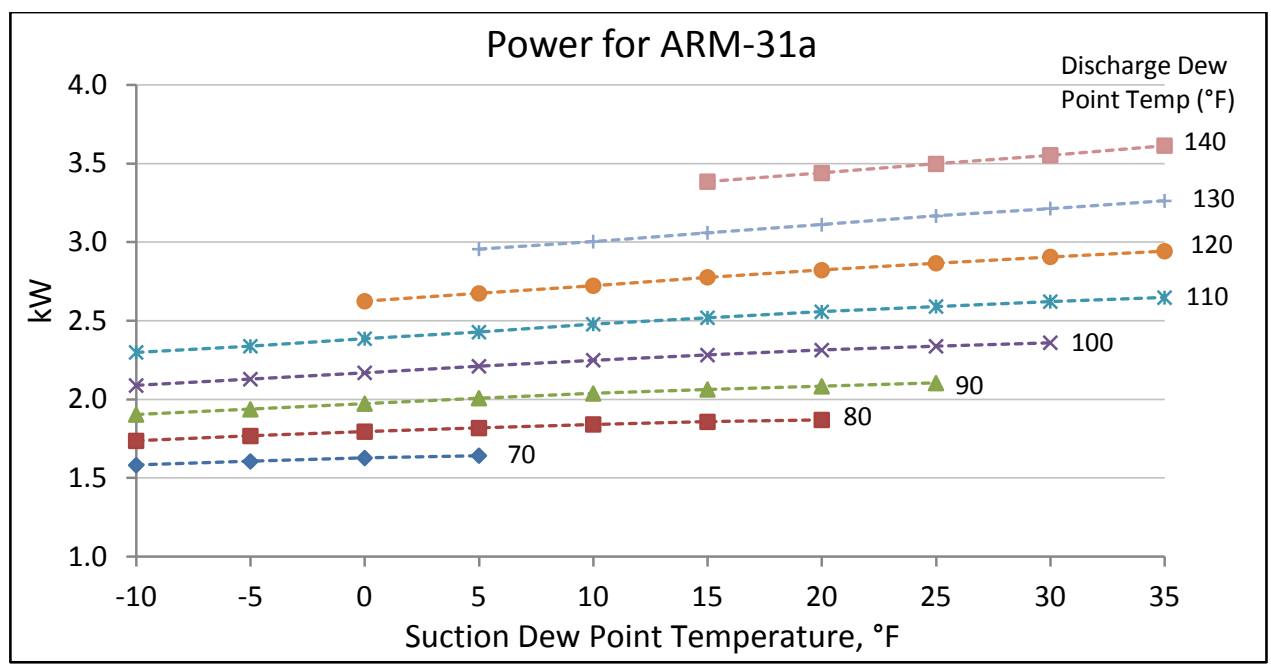

Figure B7 Power for ARM-31a. 


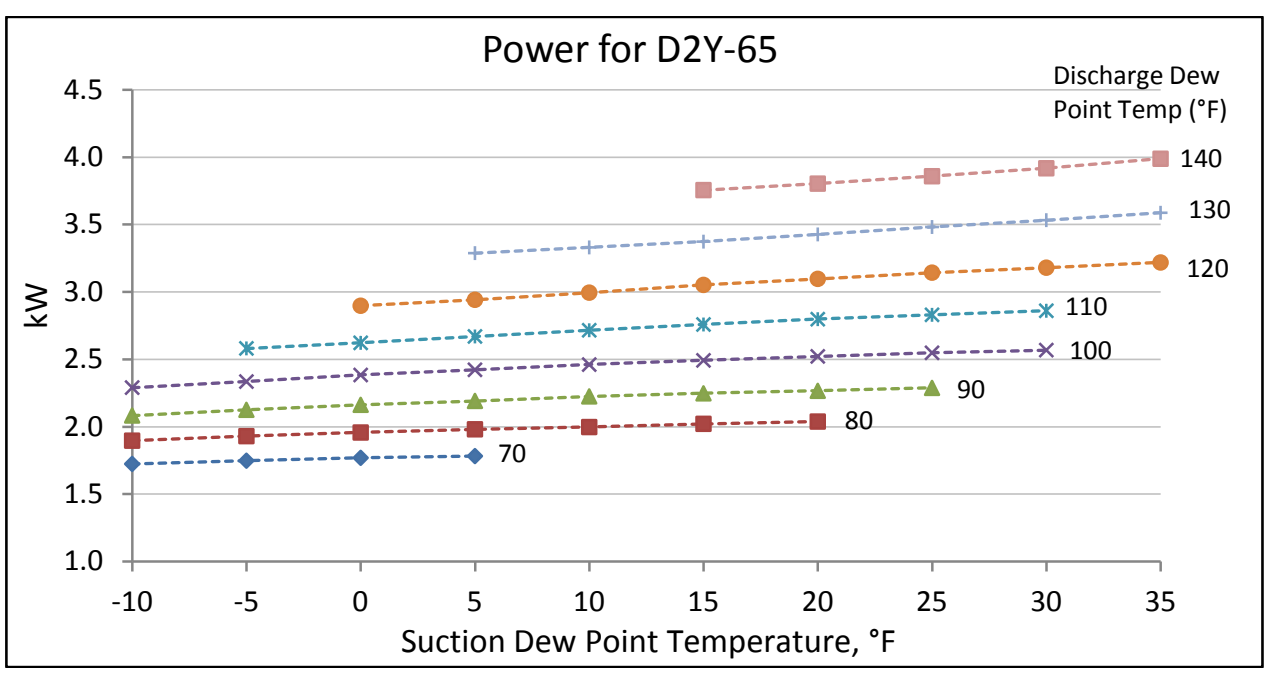

Figure B8 Power for D2Y-65.

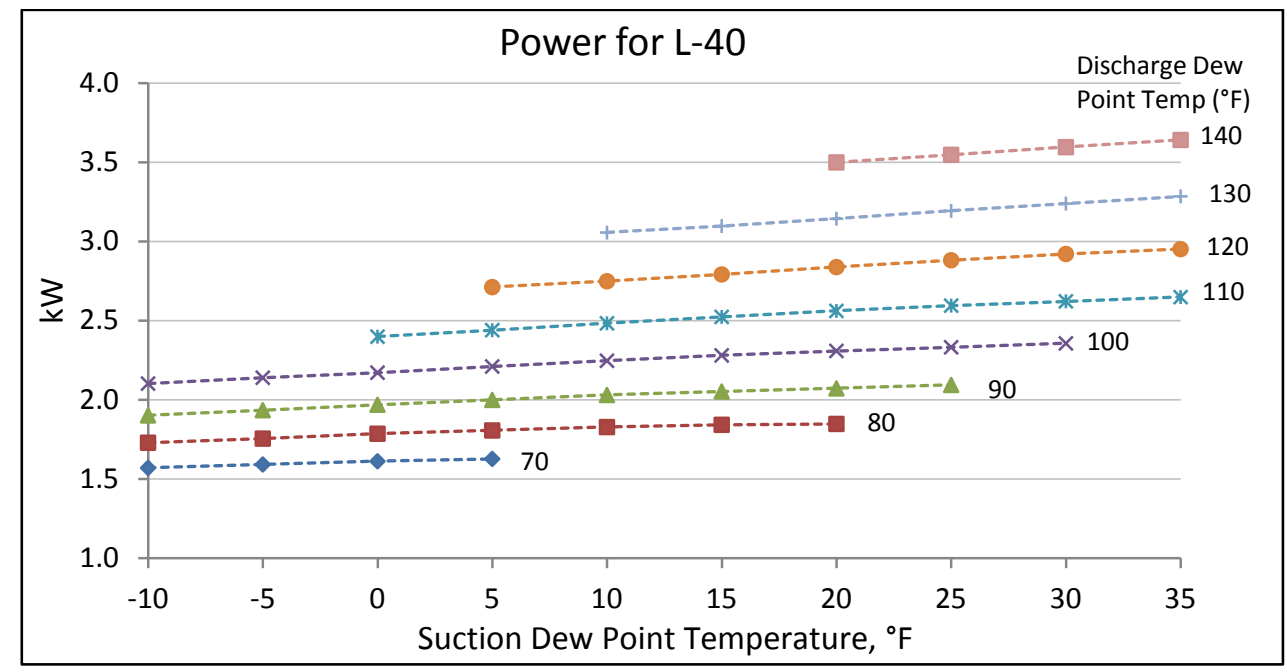

Figure B9 Power for L-40. 


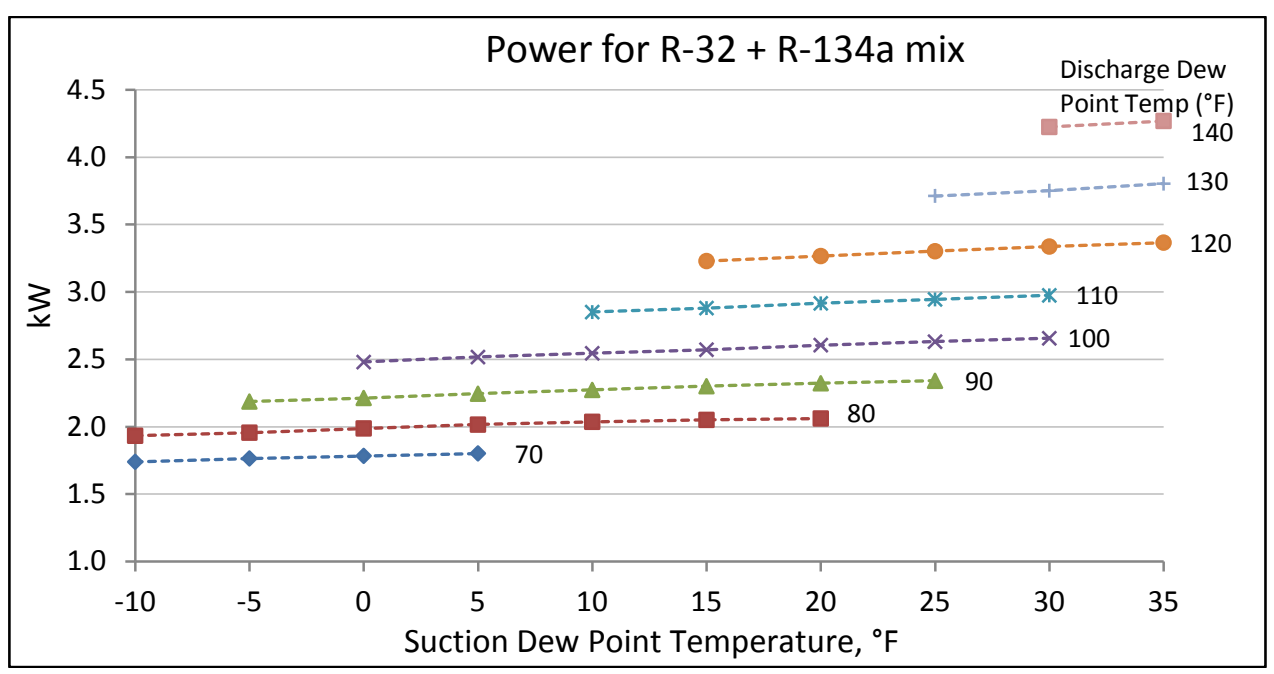

Figure B10 Power for R-32 + R-134a mixture.

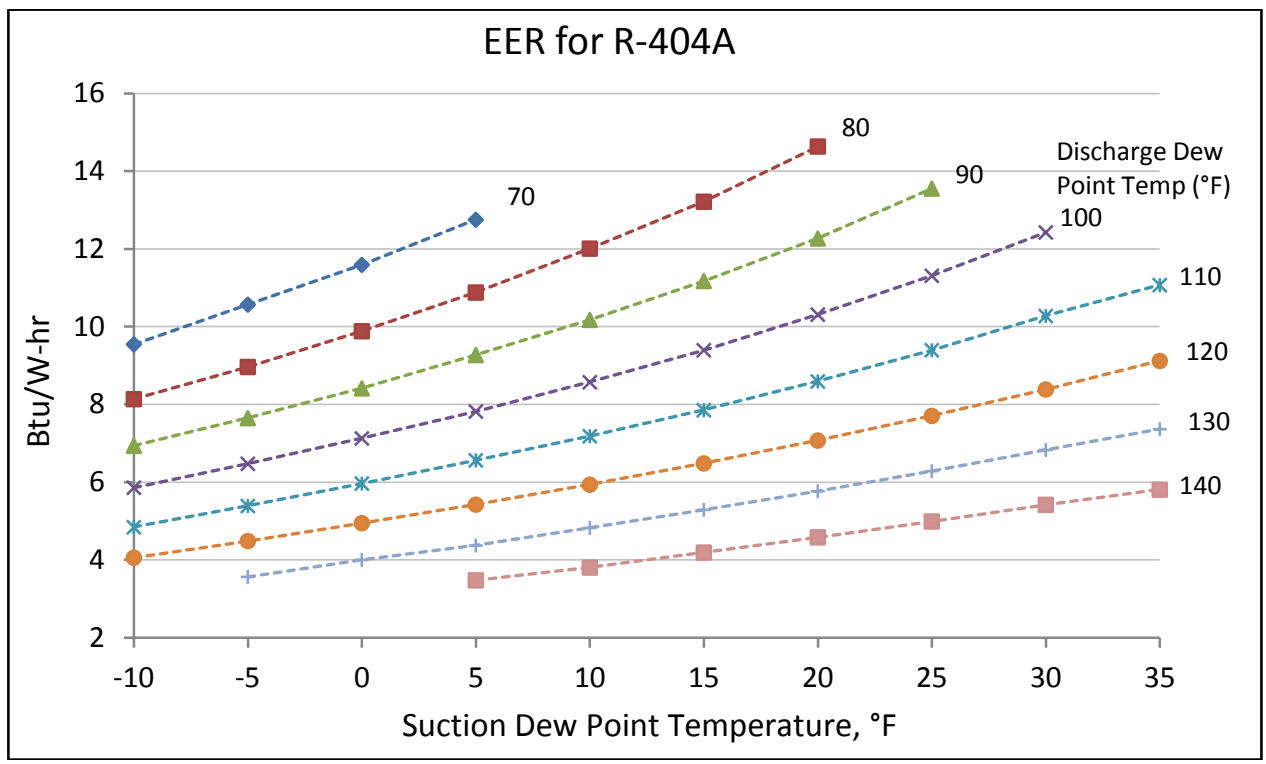

Figure B11 EER for R-404A. 


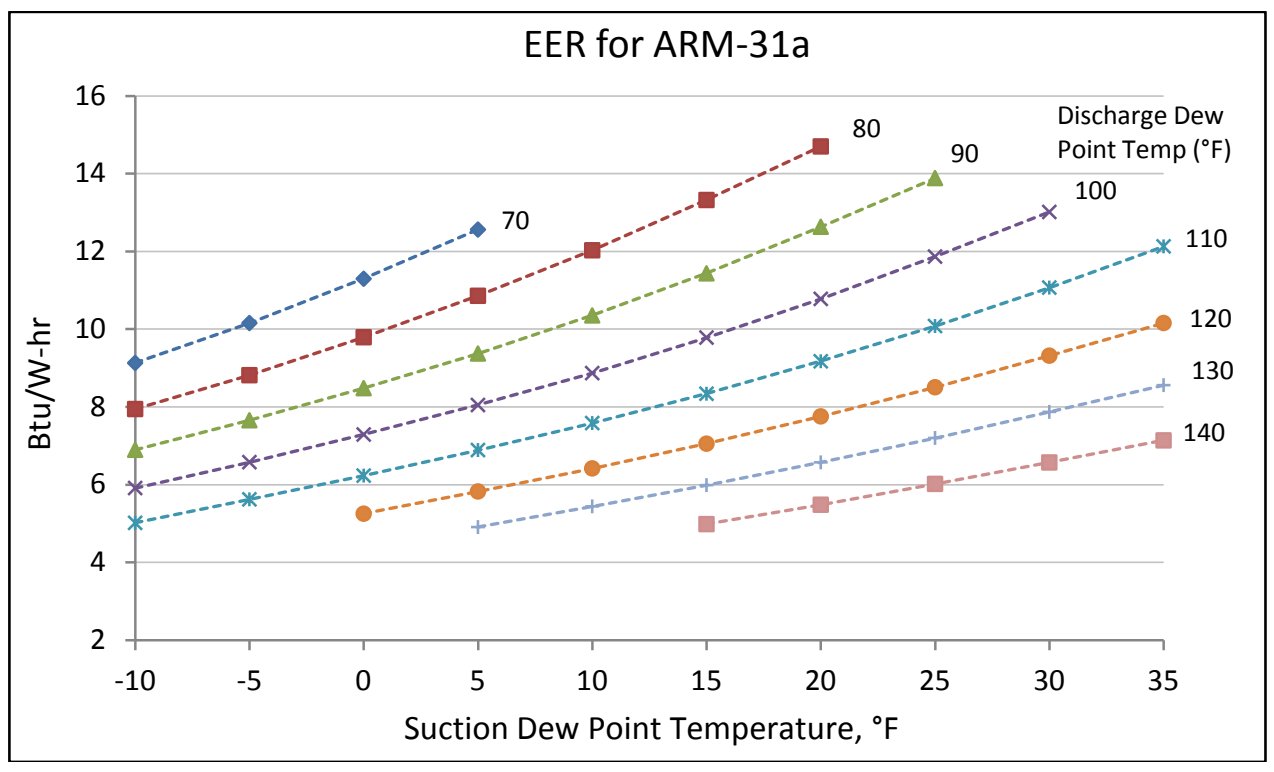

Figure B12 EER for ARM-31a.

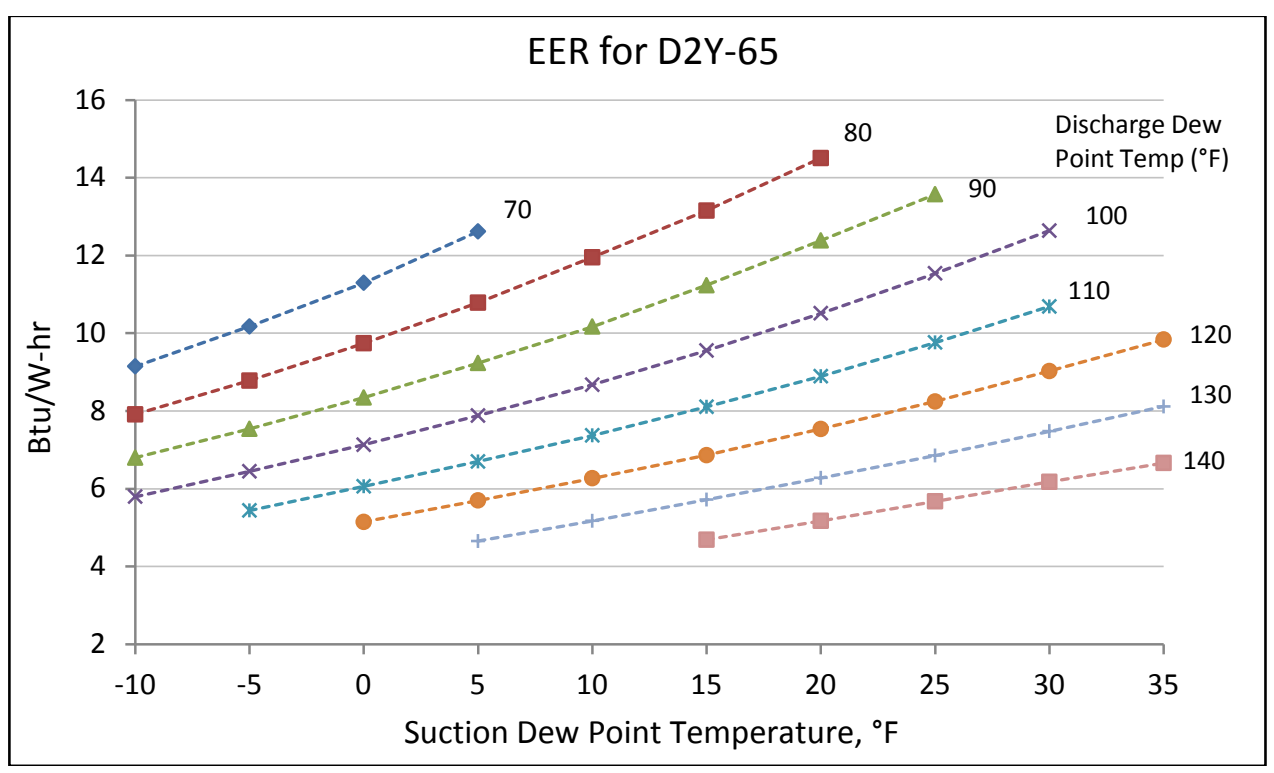

Figure B13 EER for D2Y-65. 


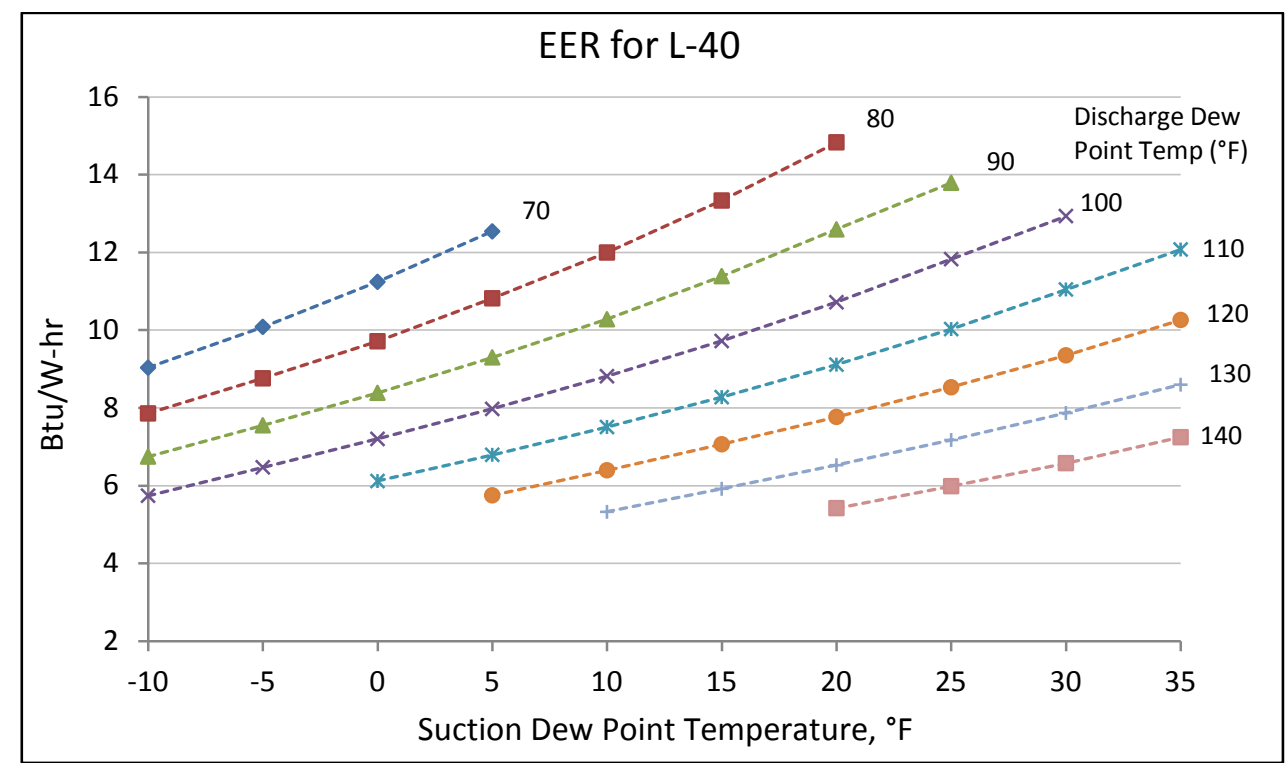

Figure B14 EER for L-40.

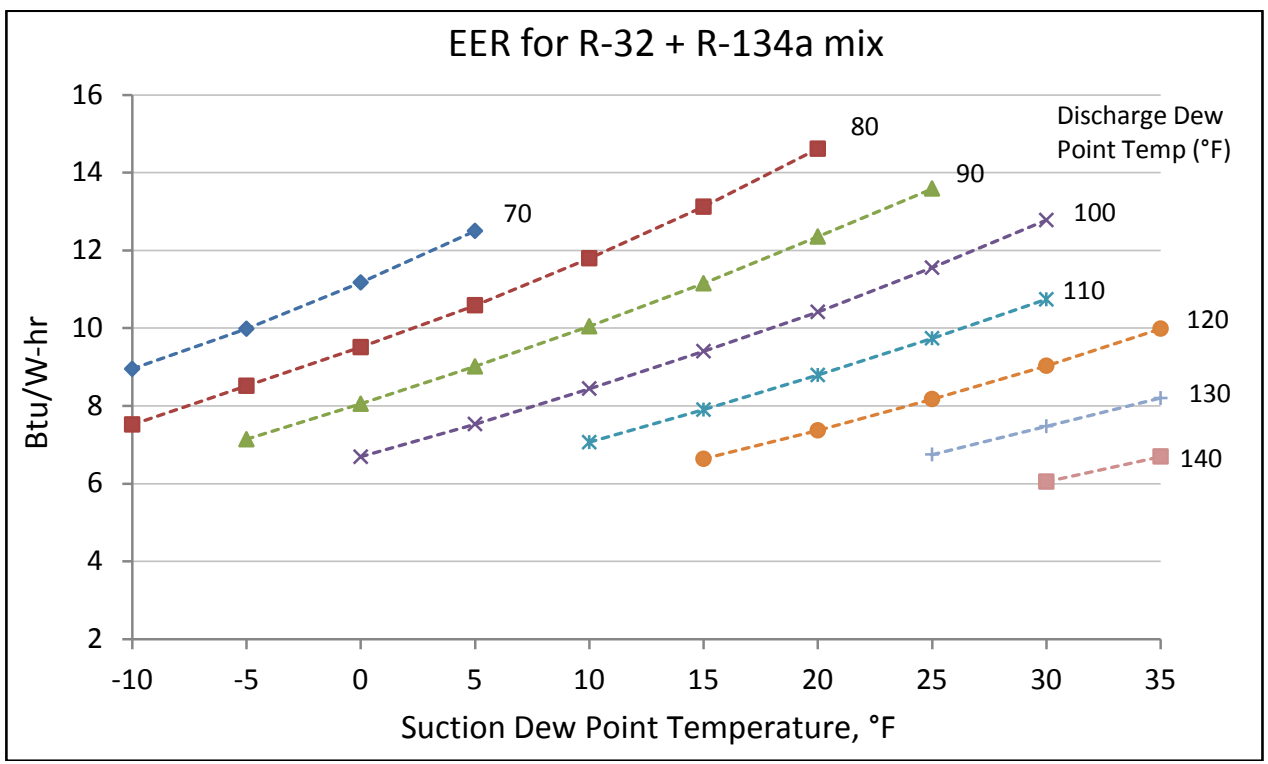

Figure B15 EER for R-32 + R-134a mixture. 


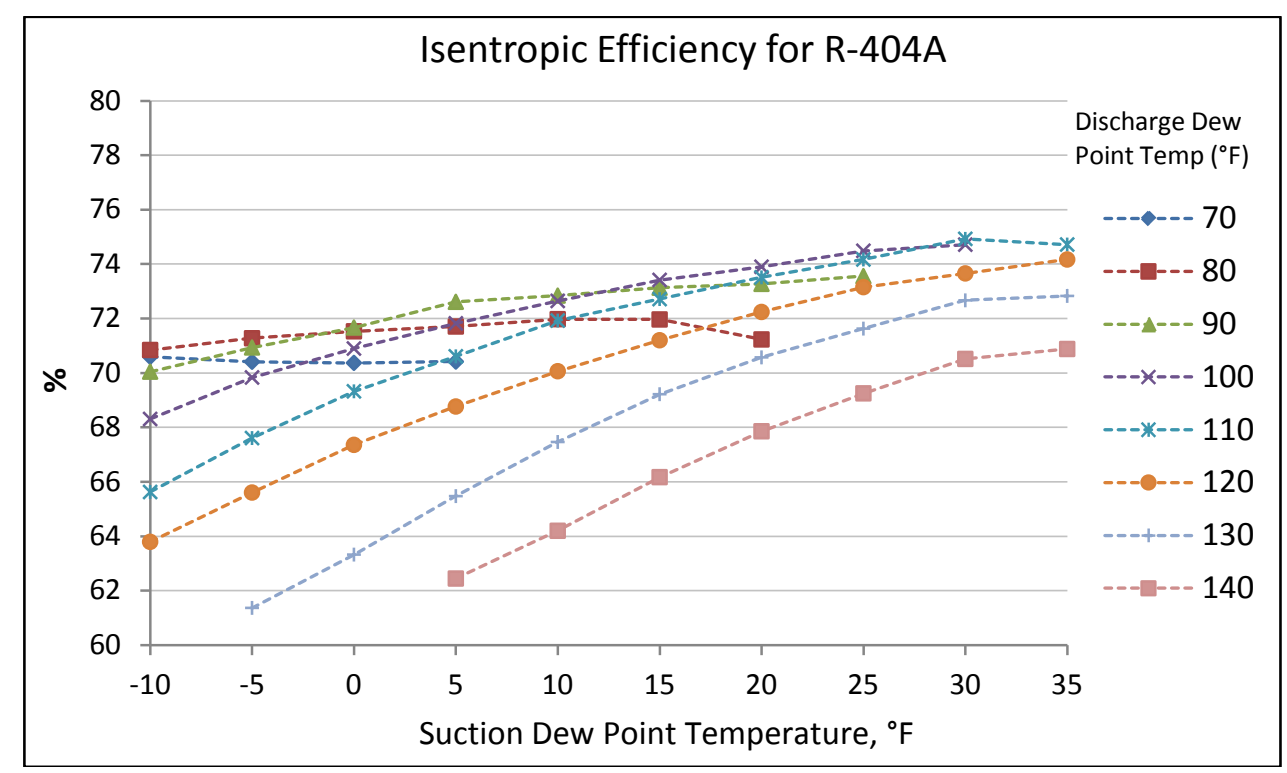

Figure B16 Isentropic efficiency for R-404A.

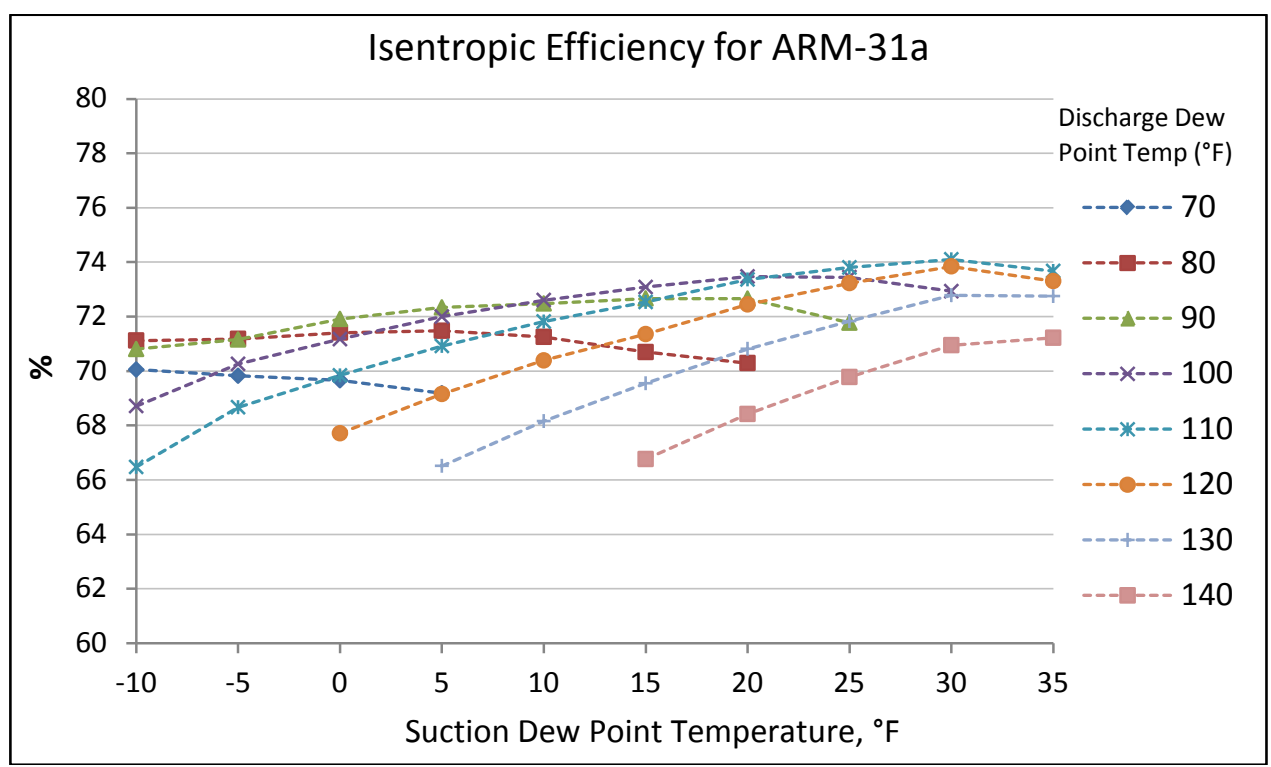

Figure B17 Isentropic efficiency for ARM-31a. 


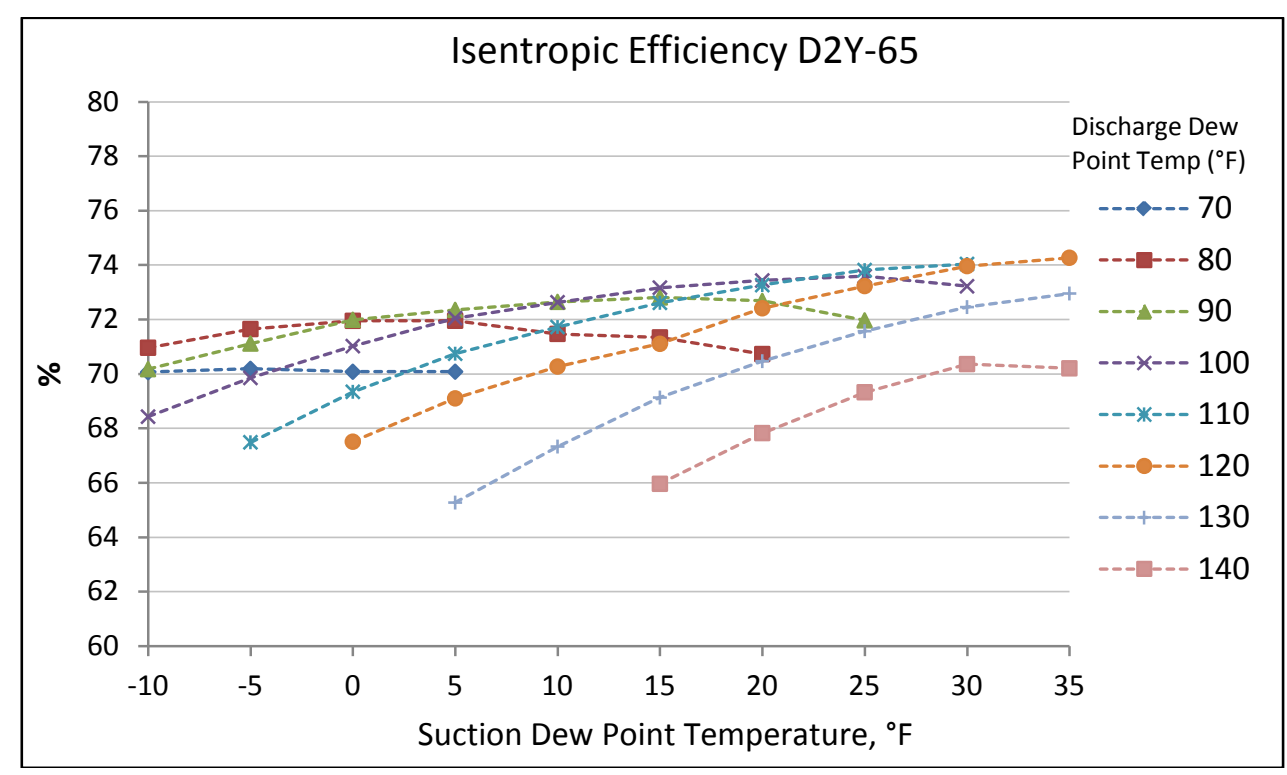

Figure B18 Isentropic efficiency for D2Y-65.

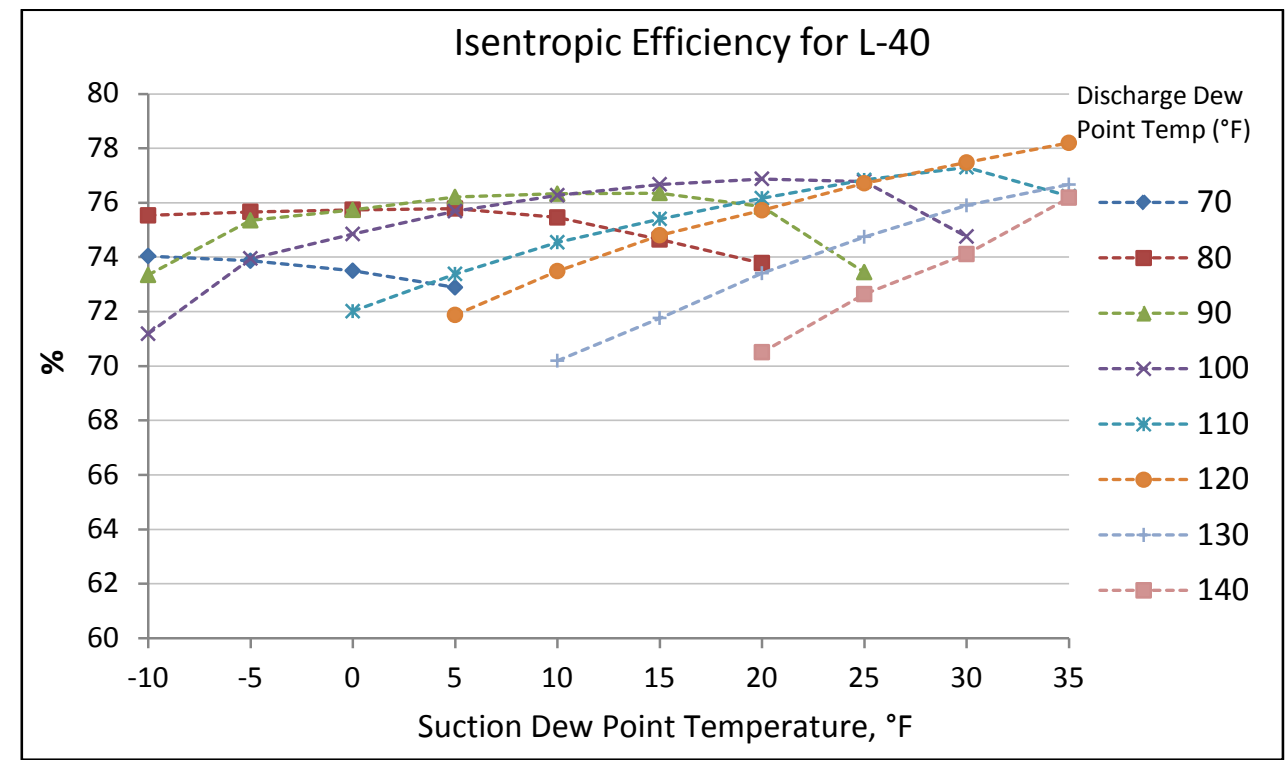

Figure B19 Isentropic efficiency for L-40. 


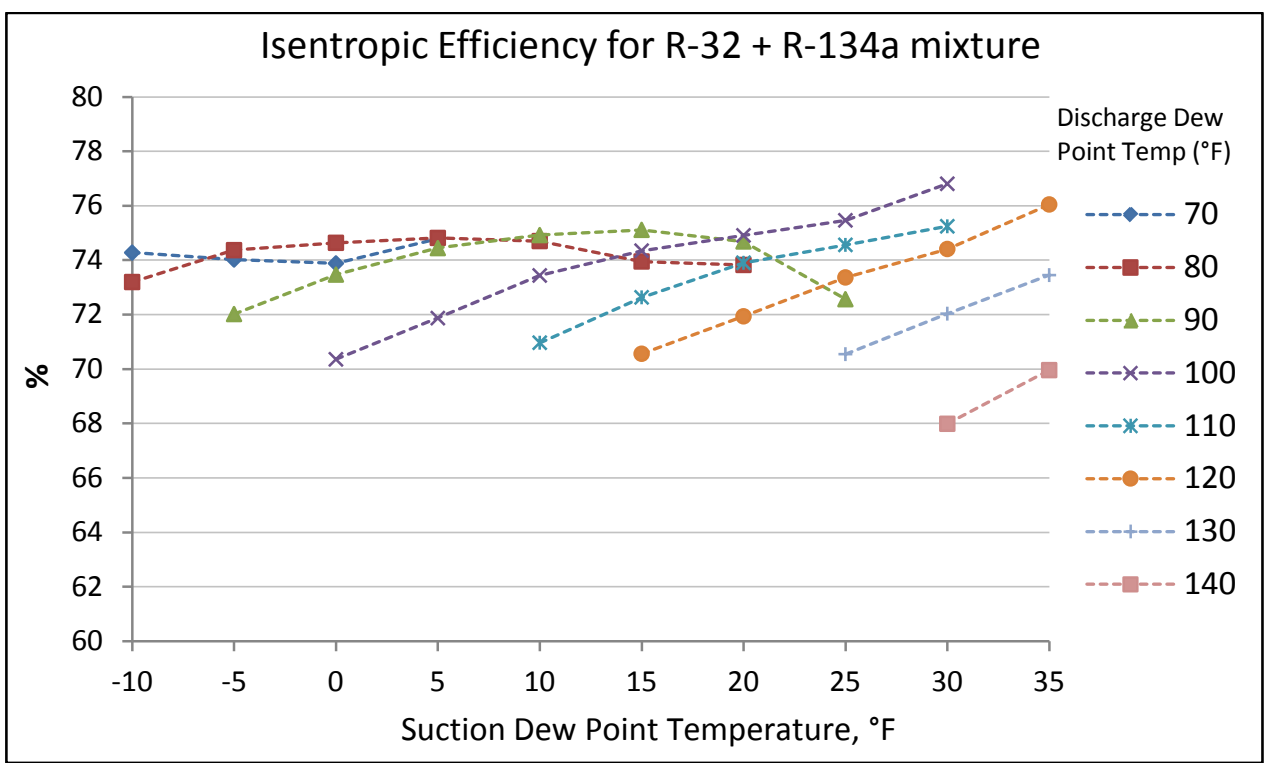

Figure B20 Isentropic efficiency for R-32 + R-134a mixture.

\section{- 10-Coefficient polynomial equation for each test refrigerant}

Tables B1 to B5 provide the 10 coefficients for mass flow rate, capacity, power, EER, and discharge temperature polynomials for each refrigerant, as a function of suction dew point temperature and discharge dew point temperature. These coefficients are derived from the test data at $65^{\circ} \mathrm{F}$ suction temperature and $0^{\circ} \mathrm{F}$ subcooling. The standard form of the 10-coefficient polynomial is given as follows:

$$
\begin{aligned}
X= & C_{1}+C_{2} * S+C_{3} * D+C_{4} * S^{2}+C_{5} * S * D+C_{6} * D^{2}+C_{7} * S^{3} \\
& +C_{8} * D * S^{2}+C_{9} * S * D^{2}+C_{10} * D^{3}
\end{aligned}
$$

Where:

$\mathrm{C}=$ Equation coefficient, represents compressor performance

$\mathrm{S}=$ Suction dew point temperature. ${ }^{\circ} \mathrm{F}$

$\mathrm{D}=$ Discharge dew point temperature. ${ }^{\circ} \mathrm{F}$

$\mathrm{X}=$ Compressor performance (mass flow rate, capacity, power, EER, discharge temperature) 
Table B1: 10 Coefficients for R-404A

\begin{tabular}{|c|c|c|c|c|c|}
\hline Coefficient & $\begin{array}{c}\text { Mass Flow, } \\
\text { Ibs/hr }\end{array}$ & $\begin{array}{c}\text { Capacity, } \\
\text { Btu/hr }\end{array}$ & Power, W & $\begin{array}{c}\text { EER, } \\
\text { Btu/W-hr }\end{array}$ & $\begin{array}{c}\text { Discharge } \\
\text { Temperature, }\end{array}$ \\
\hline \hline$C_{1} \mathrm{~F}$
\end{tabular}

Table B2: 10 Coefficients for ARM-31a

\begin{tabular}{|c|c|c|c|c|c|}
\hline Coefficient & $\begin{array}{c}\text { Mass Flow, } \\
\text { Ibs/hr }\end{array}$ & $\begin{array}{c}\text { Capacity, } \\
\text { Btu/hr }\end{array}$ & Power, W & $\begin{array}{c}\text { EER, } \\
\text { Btu/W-hr }\end{array}$ & $\begin{array}{c}\text { Discharge } \\
\text { Temperature, }\end{array}$ \\
\hline \hline$C_{1} \mathrm{~F}$
\end{tabular}


Table B3: 10 Coefficients for D2Y-65

\begin{tabular}{|c|c|c|c|c|c|}
\hline Coefficient & $\begin{array}{c}\text { Mass Flow, } \\
\mathrm{lbs} / \mathrm{hr}\end{array}$ & $\begin{array}{c}\text { Capacity, } \\
\text { Btu/hr }\end{array}$ & Power, W & $\begin{array}{c}\mathrm{EER}, \\
\text { Btu/W-hr }\end{array}$ & $\begin{array}{c}\text { Discharge } \\
\text { Temperature, }\end{array}{ }^{\circ} \mathrm{F}$ \\
\hline \hline $\mathrm{C}_{1}$ & $2.836 \mathrm{E}+02$ & $3.022 \mathrm{E}+04$ & $-1.242 \mathrm{E}+02$ & $3.434 \mathrm{E}+01$ & $9.208 \mathrm{E}+01$ \\
\hline $\mathrm{C}_{2}$ & $5.828 \mathrm{E}+00$ & $6.132 \mathrm{E}+02$ & $-2.490 \mathrm{E}+01$ & $7.155 \mathrm{E}-01$ & $-2.418 \mathrm{E}+00$ \\
\hline$C_{3}$ & $-1.849 \mathrm{E}+00$ & $-2.130 \mathrm{E}+02$ & $4.246 \mathrm{E}+01$ & $-5.268 \mathrm{E}-01$ & $1.669 \mathrm{E}+00$ \\
\hline$C_{4}$ & $6.560 \mathrm{E}-02$ & $7.160 \mathrm{E}+00$ & $-3.695 \mathrm{E}-01$ & $5.467 \mathrm{E}-03$ & $-1.497 \mathrm{E}-02$ \\
\hline$C_{5}$ & $-1.450 \mathrm{E}-02$ & $-2.341 \mathrm{E}+00$ & $5.711 \mathrm{E}-01$ & $-9.296 \mathrm{E}-03$ & $2.258 \mathrm{E}-02$ \\
\hline$C_{6}$ & $1.893 \mathrm{E}-02$ & $1.326 \mathrm{E}+00$ & $-3.278 \mathrm{E}-01$ & $3.492 \mathrm{E}-03$ & $-8.719 \mathrm{E}-03$ \\
\hline$C_{7}$ & $4.389 \mathrm{E}-04$ & $2.389 \mathrm{E}-02$ & $-1.397 \mathrm{E}-03$ & $1.564 \mathrm{E}-05$ & $-1.881 \mathrm{E}-04$ \\
\hline$C_{8}$ & $-2.102 \mathrm{E}-04$ & $-3.973 \mathrm{E}-02$ & $3.391 \mathrm{E}-03$ & $-4.482 \mathrm{E}-05$ & $3.507 \mathrm{E}-04$ \\
\hline$C_{9}$ & $8.741 \mathrm{E}-05$ & $2.311 \mathrm{E}-03$ & $-2.377 \mathrm{E}-03$ & $3.559 \mathrm{E}-05$ & $-2.195 \mathrm{E}-04$ \\
\hline$C_{10}$ & $-7.123 \mathrm{E}-05$ & $-5.177 \mathrm{E}-03$ & $1.536 \mathrm{E}-03$ & $-9.443 \mathrm{E}-06$ & $5.704 \mathrm{E}-05$ \\
\hline
\end{tabular}

Table B4: 10 Coefficients for L-40

\begin{tabular}{|c|c|c|c|c|c|}
\hline Coefficient & $\begin{array}{c}\text { Mass Flow, } \\
\text { Ibs/hr }\end{array}$ & $\begin{array}{c}\text { Capacity, } \\
\text { Btu/hr }\end{array}$ & Power, W & $\begin{array}{c}\text { EER, } \\
\text { Btu/W-hr }\end{array}$ & $\begin{array}{c}\text { Discharge } \\
\text { Temperature, }{ }^{\circ} \mathrm{F}\end{array}$ \\
\hline \hline $\mathrm{C}_{1}$ & $1.956 \mathrm{E}+02$ & $2.434 \mathrm{E}+04$ & $7.809 \mathrm{E}+01$ & $3.234 \mathrm{E}+01$ & $1.326 \mathrm{E}+02$ \\
\hline $\mathrm{C}_{2}$ & $4.965 \mathrm{E}+00$ & $5.720 \mathrm{E}+02$ & $-2.279 \mathrm{E}+01$ & $7.407 \mathrm{E}-01$ & $-2.086 \mathrm{E}+00$ \\
\hline$C_{3}$ & $-6.128 \mathrm{E}-01$ & $-1.077 \mathrm{E}+02$ & $3.334 \mathrm{E}+01$ & $-4.776 \mathrm{E}-01$ & $5.810 \mathrm{E}-01$ \\
\hline$C_{4}$ & $4.285 \mathrm{E}-02$ & $5.664 \mathrm{E}+00$ & $-3.688 \mathrm{E}-01$ & $5.702 \mathrm{E}-03$ & $1.945 \mathrm{E}-03$ \\
\hline$C_{5}$ & $-1.537 \mathrm{E}-02$ & $-2.115 \mathrm{E}+00$ & $5.530 \mathrm{E}-01$ & $-9.813 \mathrm{E}-03$ & $1.311 \mathrm{E}-02$ \\
\hline$C_{6}$ & $6.863 \mathrm{E}-03$ & $4.378 \mathrm{E}-01$ & $-2.543 \mathrm{E}-01$ & $3.129 \mathrm{E}-03$ & $2.927 \mathrm{E}-03$ \\
\hline$C_{7}$ & $2.891 \mathrm{E}-04$ & $1.694 \mathrm{E}-02$ & $-2.139 \mathrm{E}-03$ & $1.693 \mathrm{E}-05$ & $-1.486 \mathrm{E}-04$ \\
\hline$C_{8}$ & $-3.857 \mathrm{E}-05$ & $-2.336 \mathrm{E}-02$ & $3.875 \mathrm{E}-03$ & $-4.763 \mathrm{E}-05$ & $1.969 \mathrm{E}-04$ \\
\hline$C_{9}$ & $8.369 \mathrm{E}-05$ & $2.218 \mathrm{E}-03$ & $-2.517 \mathrm{E}-03$ & $3.910 \mathrm{E}-05$ & $-1.790 \mathrm{E}-04$ \\
\hline$C_{10}$ & $-3.213 \mathrm{E}-05$ & $-2.310 \mathrm{E}-03$ & $1.304 \mathrm{E}-03$ & $-8.669 \mathrm{E}-06$ & $2.182 \mathrm{E}-05$ \\
\hline
\end{tabular}


Table B5: 10 Coefficients for R-32 + R-134a mixture

\begin{tabular}{|c|c|c|c|c|c|}
\hline Coefficient & $\begin{array}{c}\text { Mass Flow, } \\
\text { Ibs/hr }\end{array}$ & $\begin{array}{c}\text { Capacity, } \\
\text { Btu/hr }\end{array}$ & Power, W & $\begin{array}{c}\text { EER, } \\
\text { Btu/W-hr }\end{array}$ & $\begin{array}{c}\text { Discharge } \\
\text { Temperature, }\end{array}$ \\
\hline \hline$C_{1} \mathrm{~F}$
\end{tabular}

